\title{
ONE-DIMENSIONAL NIL-DAHA AND WHITTAKER FUNCTIONS
}

\author{
IVAN CHEREDNIK ${ }^{\dagger}$ AND DANIEL ORR
}

\begin{abstract}
This paper is devoted to the theory of nil-DAHA for the root system $A_{1}$ and its applications to symmetric and nonsymmetric (spinor) global $q$-Whittaker functions. These functions integrate the $q$-Toda eigenvalue problem and its Dunkl-type nonsymmetric version.

The global symmetric function can be interpreted as the generating function of the Demazure characters for dominant weights, which describe the algebraic-geometric properties of the corresponding affine Schubert varieties. Its Harish-Chandra-type asymptotic expansion appeared directly related to the solution of the $q$-Toda eigenvalue problem obtained by Givental and Lee in the quantum $K$-theory of flag varieties. It provides an exact mathematical relation between the corresponding physics $A$-type and $B$-type models.

The spinor global functions extend the symmetric ones to the case of all Demazure characters (not only those for the dominant weights); the corresponding Gromov-Witten theory is not known. The main result of the paper is a complete algebraic theory of these functions in terms of the induced modules of the core subalgebra of nil-DAHA. It is the first instance of the DAHA theory of canonicalcrystal bases, quite non-trivial even for $A_{1}$.
\end{abstract}

\section{Contents}

0. INTRODUCTION 4

1. Polynomial RePresentation 7

1.1. Main definitions $\quad 7$

1.1.1. Automorphisms 8

1.1.2. Inner products $\quad 9$

1.2. The E-polynomials 9

1.2.1. The intertwiners 10

1.2.2. The E-Pieri rules 11

$\begin{array}{ll}1.2 .3 . & \text { Rogers' polynomials } \\ 12\end{array}$

$\begin{array}{ll}\text { 1.2.4. Explicit formulas } & 12\end{array}$

$\dagger \quad$ Partially supported by NSF grant DMS-1101535.

This paper will appear in Transformation Groups in two parts. 
2. Global FunCtions 13

2.1. Spherical functions 13

2.1.1. Gauss-type integrals $\quad 13$

2.1.2. Fourier transforms 14

$\begin{array}{ll}\text { 2.1.3. Reproducing kernels } & 15\end{array}$

2.1.4. Nonsymmetric global functions 16

$\begin{array}{ll}2.2 \text {. Whittaker functions } & 18\end{array}$

2.2.1. Whittaker limit $\quad 19$

2.2.2. Harish-Chandra expansion 21

2.2.3. Nonsymmetric q-Hermite polynomials 25

2.2.4. The series for the mu-function 27

3. NIL-DAHA 28

3.1. Key definitions 28

3.1.1. Polynomial representation $\quad 29$

3.1.2. The order function 30

3.1.3. Invariant symmetric forms 30

3.2. Using the conjugation 31

3.2.1. The limit at infinity 31

3.2.2. Connection maps $\quad 32$

3.2.3. Pairing with conjugation 33

3.3. Tilde-subalgebras 34

3.3.1. Alternative presentations $\quad 34$

3.3.2. Transitional subalgebras $\quad 35$

3.3.3. Defining relations 35

3.4. PBW and filtrations 36

3.4.1. Using the order 38

4. INDUCED REPRESENTATIONS 39

4.1. Tilde-polynomial modules 39

4.1.1. Tilde-induction $\quad 39$

4.1.2. Explicit identification $\quad 40$

4.1.3. Basic operators 41

4.2. The core subalgebra 42

4.2.1. Tilde-intertwiners $\quad 45$

4.2.2. Dual induced modules 48

4.2.3. Decomposition 49

4.3. Shapovalov forms 51

4.3.1. Shapovalov trace $\quad 51$

4.3.2. Shapovalov pairing 53

4.3.3. The tilde-case 53

4.3.4. Induction from tilde-X 54

5. SPINORS AND THE $q$-TODA THEORY 55

5.1. The spinors $\quad 55$ 
5.1.1. The definitions $\quad 56$

5.1.2. Spinor operators $\quad 56$

5.1.3. The q-Toda operators via DAHA 56

5.2. Spinor Whittaker function 58

5.2.1. Basic operators $\quad 58$

5.2.2. Using the components $\quad 59$

5.2.3. The main formula 60

5.3. Algebraic theory 61

5.3.1. Relation to tilde-modules 61

5.3.2. Algebraic Whittaker function 63

5.3.3. Justification 64

5.3.4. Omitting the conjugation 66

6. THE CASE OF LARGE $q \quad 67$

6.1. Omega-check function $\quad 67$

6.1.1. Twisted RE-procedure $\quad 67$

6.1.2. Definition 68

6.1.3. Symmetrization 68

6.1.4. Discussion $\quad 69$

6.2. Main theorem $\quad 69$

6.2.1. Proof 70

6.3. Basic operators (large q) 72

$\begin{array}{lll}\text { 6.3.1. T-check operator } & 72\end{array}$

6.3.2. Y-check operators $\quad 73$

6.3.3. The symmetrization 73

6.3.4. Pi-check operator $\quad 74$

6.4. The eigenvalue problem 75

$\begin{array}{ll}\text { 6.4.1. Preparations } & 75\end{array}$

6.4.2. Explicit verification 76

7. Appendix: Givental-Lee Theory 77

$\begin{array}{ll}\text { 7.1. The J-series } & 77\end{array}$

$\begin{array}{ll}\text { 7.2. The connection } & 78\end{array}$

$\begin{array}{ll}\text { REFERENCES } & 79\end{array}$ 


\section{INTRODUCTION}

This paper is devoted to the theory of nil-DAHA $\overline{\mathcal{H}}$ for the root system $A_{1}$ and its applications to the symmetric and nonsymmetric qWhittaker functions, integrating the $q$-Toda eigenvalue problem and its Dunkl counterpart introduced in [ChM].

In the symmetric case, we establish an exact relation between the generating functions of the $A$-type model ( $K$-theoretic Gromov-Witten invariants of flag varieties) and the $B$-type model (sheaf cohomology of affine Schubert varieties) in the case of $S L(2)$.

Major connections. In greater detail, the global symmetric $q-$ Whittaker functions, defined in [Ch5] for any reduced root systems, can be interpreted as generating functions of level one Demazure characters (equivalently, $q$-Hermite polynomials) for dominant weights; these characters describe the spaces of global sections of the canonical level one line bundle restricted to the corresponding affine Schubert subvarieties of the affine flag variety. From this perspective, the symmetric $q$-Whittaker functions are examples of the generating functions of $B-$ type models in physics.

On the other hand, the Harish-Chandra asymptotic decompositions of these functions from [Ch5] are directly related to the solutions of the $q$-Toda eigenvalue problem found in [GiL]. These solutions are important instances of generating functions for $A$-models, as they describe $K$-theoretic genus zero Gromov-Witten invariants. The Appendix, Section 7, contains more details on this connection.

Thus we arrive at an exact mathematical relation between these two theories. It is described in this paper (mathematically) in full detail for type $A_{1}$. This construction is a explicit example of the Whittaker limit of the $q, t$-generalization of Harish-Chandra theory of asymptotic expansions started in [Ch5] and recently finalized in [Sto2].

Nonsymmetric functions. One of the main results of $[\mathrm{ChM}]$ is the construction of the spinor Dunkl operators for the $q$-Toda eigenvalue problem in type $A_{1}$. It was a surprising development, since the $q$-Toda operators are already nonsymmetric and the standard approach based on the symmetrization of the Dunkl operators was not expected to work.

A related development of $[\mathrm{ChM}]$ is the definition of the nonsymmetric $q$-Whittaker function, which integrates the eigenvalue problem for the spinor Dunkl operators. A variant of the Ruijsenaars-Etingof limiting procedure [Rui, Et] was used to obtain it from the nonsymmetric global $q, t$-spherical function defined in [Ch3]. 
In the $B$-type model interpretation, the nonsymmetric $q$-Whittaker function extends its symmetric counterpart to the case of all Demazure characters (not only those for the dominant weights) and arbitrary Schubert subvarieties of the affine flag variety. The "nonsymmetric" counterpart of the $K$-theoretic Gromov-Witten theory is not known.

The natural expectations are that the nonsymmetric $q$-Whittaker functions will impact the classical real and $p$-adic theories of Whittaker functions, as well as the recent Whittaker theories based on the Kac-Moody algebras and Quantum Groups. See [ChM], [GiL], [GLO], $[\mathrm{FJM}]$, and $[\mathrm{BF}]$ for this and other known and expected applications of $q$-Whittaker functions. In this connection, let us also mention that the nil-DAHA is directly related to the constructions of [KK] in the case of the affine flag variety.

The Matsumoto $p$-adic spherical functions, the simplest example of the nonsymmetric theory, demonstrate potential power of such development. DAHA already resulted in the theory of real counterparts of the Matsumoto functions, which are the nonsymmetric Macdonald polynomials, and unified the real and $p$-adic theories in one $q, t$-theory. Now it can be done for Whittaker functions!

Core subalgebra. The main result of this paper is an entirely algebraic theory of the spinor Dunkl operators and nonsymmetric Whittaker function for $A_{1}$ in terms of the induced modules of a new object, the core subalgebra of nil-DAHA.

The core subalgebra is essentially the span of the basic creation and annihilation operators for the nonsymmetric $q$-Hermite polynomials and the $Y$-operators (which diagonalize these polynomials). Its existence as a subalgebra of $\overline{\mathcal{H}}$ is an important special feature of the nil-case. Conceptually, the nonsymmetric Whittaker function is the reproducing kernel of the transform sending the nonsymmetric q-Hermite polynomials to the corresponding creation operators. See Theorem 4.8, a simple (at least in $A_{1}$ ) but fundamental fact; this connection seems to be the main algebraic message of this paper.

It is expected that the core subalgebra will play the prime role in the theory of canonical-crystal bases of DAHA. Its graded algebra is a nil-nil variant of the extended Weyl algebra (a non-commutative torus extended by the Weyl group $W$ ), which is quite interesting in its own right.

This paper contains a systematic analysis of modules for the core subalgebra induced from one-dimensional representations of core affine Hecke subalgebras. Some of these modules totally collapse, i.e., remain one-dimensional. The induced modules that are of the right size 
(infinite-dimensional, of course) can be extended to modules over the whole $\overline{\mathcal{H}}$. This is one of the key results of the paper, Theorem 4.3. The core-induced modules have a natural filtration of submodules; the corresponding irreducible constituents are one-dimensional (for $A_{1}$ ) and can be calculated in full detail.

A key point is that the core subalgebra contains all intertwining operators necessary to decompose such and similar induced modules. It is an important change from the theory of intertwiners for AHA and DAHA. Actually, it is a new and interesting version of the technique of intertwiners in the nil-case.

The core subalgebra solves the problem of finding an induced interpretation of the spinor-polynomial module. This module was discovered in $[\mathrm{ChM}]$ analytically, via reading the coefficients of the spinor $q$-Whittaker function, obtained there as a limit of the global spherical nonsymmetric function. Now we can introduce it entirely algebraically; it is isomorphic to an induced module of the core subalgebra upon its extension to $\overline{\mathcal{H}}$. Correspondingly, the spinor $q-$ Whittaker function can be now defined and calculated within $\overline{\mathcal{H}}$, i.e., without any reference to the $q, t$-theory. See Theorems 5.3 and 5.4.

Analytic aspects. There nonsymmetric global spherical functions defined in [Ch3] come in two variants, one for $|q|<1$ and one for $|q|>1$. We note that these functions are quite different, though there is a connection. Both are needed in the analytic theory, for instance, to obtain the theory for all $q$, including the unit circle.

The nonsymmetric Whittaker function defined in [ChM] corresponds to $|q|<1$. The case $|q|>1$ appeared more involved; in fact, the limiting procedure must be twisted. In this paper, we define the appropriate limiting procedure and use it to produce the nonsymmetric Whittaker function for $|q|>1$.

Interestingly, in contrast to the $q, t$-case, both nonsymmetric $q-$ Whittaker functions can be extended to all $|q| \neq 1$ and almost all $q$ at the unit circle. However the relation between these two functions becomes more sophisticated than in the $q, t$-case, since they correspond to different limits, namely $t \rightarrow 0$ and $t \rightarrow \infty$ (and they solve different equations).

In the symmetric setting, the Whittaker functions are connected by a simple conjugation sending $q$ to $q^{-1}$. Theorem 2.2 below establishes the analogue of this connection in the nonsymmetric case. The two functions extend each other through a variant of the Hardy-Littlewood continuation theory from $|q| \neq 1$ to $|q|=1$ (almost everywhere). Here roots of unity must be avoided, but not only such points. The most 
direct reference is [Lub]. It is likely that the roots of unity will be the only obstacle for such continuation in the final theory, but a different approach will be necessary.

Some other results. The paper contains a systematic algebraic theory of the nil-DAHA and its subalgebras. It includes several PBWtheorems (some are of unusual type), the theory of inner products and study of the polynomial and various induced representations. An important fact is that the core subalgebra is bi-graded and also has a natural filtration with the corresponding graded algebra that is a nil-Weyl algebra. The bi-grading and the filtration are the key simplifications over the $q, t$-case. Let us also mention Theorem 4.9, which establishes the coincidence of the bilinear quadratic form in the polynomial representation defined for the product of the Gaussian and mufunction with the simplest Shapovalov form. This relation is very deep in the $q, t$-theory. It generalizes the technique of picking residues due to Arthur-Heckman-Opdam; see [O],[Ch4].

Acknowledgements. The first author is thankful to D. Kazhdan for useful discussions, to E. Opdam, J. Stokman and N. Wallach for various talks on the Harish-Chandra and Whittaker theory. We also thank S. Kumar and R. Rimanyi for helpful conversations. The first author thanks IHES and Caltech for the hospitality.

\section{Polynomial Representation}

1.1. Main definitions. We consider only the case of $A_{1}$ in the paper. Let $\alpha=\alpha_{1}, s=s_{1}$, and $\omega=\omega_{1}$, the fundamental weight; then $\alpha=$ $\alpha_{1}=2 \omega$ and $\rho=\omega$. The extended affine Weyl group $\widehat{W}=<s, \omega>$ is generated by $s$ and the involution $\pi=\omega s$.

The double affine Hecke algebra $\mathcal{H}$ is generated by $Y=Y_{\omega_{1}}=$ $\pi T, T=T_{1}, X=X_{\omega_{1}}$ subject to the quadratic relation $\left(T-t^{1 / 2}\right)(T+$ $\left.t^{-1 / 2}\right)=0$ and the cross-relations:

$$
T X T=X^{-1}, T^{-1} Y T^{-1}=Y^{-1}, Y^{-1} X^{-1} Y X T^{2} q^{1 / 2}=1 .
$$

Using $\pi=Y T^{-1}$, the second relation becomes $\pi^{2}=1$. The field of definition will be $\mathrm{C}_{q, t} \stackrel{\text { def }}{=} \mathrm{C}\left(q^{1 / 4}, t^{1 / 2}\right)$ although $\mathrm{z}\left[q^{ \pm 1 / 4}, t^{ \pm 1 / 2}\right]$ is sufficient for many constructions; $q^{ \pm 1 / 4}$ will be mainly needed in the automorphisms $\tau_{ \pm}$below. We will frequently treat $q, t$ as numbers; then the field of definition will be $\mathrm{C}$.

It is important that $\mathcal{H}$ at $t=1$ becomes the Weyl algebra defined as the span $\langle X, Y\rangle /\left(Y^{-1} X^{-1} Y X q^{1 / 2}=1\right)$ extended by the inversion $s=T(t=1)$ sending $X \mapsto X^{-1}$ and $Y \mapsto Y^{-1}$. 
The affine Hecke subalgebra in terms of $Y$ can be written as $\mathcal{H}_{Y}=$ $\langle Y, T\rangle$.

The polynomial representation is defined as $\mathscr{X}=\mathrm{C}_{q, t}\left[X^{ \pm 1}\right]$ with $X$ acting by the multiplication. The formulas for the other generators are

$$
T=t^{1 / 2} s+\frac{t^{1 / 2}-t^{-1 / 2}}{X^{2}-1} \circ(s-1), Y=\pi T
$$

in terms of the (multiplicative) reflection $s\left(X^{n}\right)=X^{-n}$ and $\pi\left(X^{n}\right)=$ $q^{n / 2} X^{-n}$ for $n \in \mathrm{Z}$.

We will sometimes set $X=q^{x}$. Then

$$
s(x)=-x, \omega(f(x))=f(x-1 / 2), \pi=\omega s, \pi(x)=1 / 2-x .
$$

1.1.1. Automorphisms. The following map can be extended to an antiinvolution on $\mathcal{H}$ :

$$
\varphi: X \leftrightarrow Y^{-1}, T \mapsto T, q, t \mapsto q, t .
$$

The first two relations in (1.1) are obviously fixed by $\varphi$; as for the third, check that $\varphi\left(Y^{-1} X^{-1} Y X\right)=Y^{-1} X^{-1} Y X$. Switching $X$ and $Y$ can be also achieved using the involution

$$
\varepsilon: X \leftrightarrow Y, T \mapsto T^{-1}, q^{1 / 4} \mapsto q^{-1 / 4}, t^{1 / 2} \mapsto t^{-1 / 2} .
$$

The conjugation by the Gaussian $q^{x^{2}}$ preserves $\mathcal{H}$. The Gaussian obviously belongs to a completion of $\mathscr{X}$. It satisfies:

$$
\omega\left(q^{x^{2}}\right)=q^{1 / 4} X^{-1} q^{x^{2}}, \omega\left(q^{-x^{2}}\right)=q^{-1 / 4} X q^{-x^{2}} .
$$

The conjugation $A \mapsto \tau_{+}(A)=q^{x^{2}} A q^{-x^{2}}$ for $A \in \mathcal{H}$ satisfies:

$$
\tau_{+}(X)=X, \tau_{+}(T)=T, \tau_{+}(Y)=q^{-1 / 4} X Y, \tau_{+}(\pi)=q^{-1 / 4} X \pi .
$$

To see this, use that

$$
Y=\omega \circ\left(t^{1 / 2}+\frac{t^{1 / 2}-t^{-1 / 2}}{X^{-2}-1} \circ(1-s)\right) .
$$

Applying $\varphi$ we obtain an automorphism

$$
\tau_{-}=\varphi \tau_{+} \varphi, \tau_{-}(Y)=Y, \tau_{-}(T)=T, \tau_{-}(X)=q^{1 / 4} Y X .
$$

The generalized Fourier transform, corresponds to the following automorphism of $\mathcal{H} \mathcal{H}$ (it is not an involution) :

$$
\sigma(X)=Y^{-1}, \sigma(T)=T, \sigma(Y)=q^{-1 / 2} Y^{-1} X Y=X T^{2}, \sigma(\pi)=X T,
$$

$$
\sigma=\tau_{+} \tau_{-}^{-1} \tau_{+}=\tau_{-}^{-1} \tau_{+} \tau_{-}^{-1} .
$$

Check that $\sigma \tau_{+}=\tau_{-}^{-1} \sigma, \sigma \tau_{+}^{-1}=\tau_{-} \sigma$. Also,

$$
\varphi \tau_{+}=\tau_{-} \varphi, \varphi \sigma=\sigma^{-1} \varphi, \varepsilon \tau_{+}=\tau_{-} \varepsilon, \varepsilon \sigma=\sigma^{-1} \varepsilon .
$$


Due to the group nature of the definition of $\mathcal{H}$, we have the inversion anti-involution $\mathcal{H} \mathcal{H} \ni H \mapsto H^{*}$ :

$$
X^{*}=X^{-1}, Y^{*}=Y^{-1}, T^{*}=T^{-1},\left(q^{1 / 4}\right)^{*}=q^{-1 / 4},\left(t^{1 / 2}\right)^{*}=t^{-1 / 2} .
$$

It commutes with all automorphisms and anti-automorphisms of $\mathcal{H}$. Note that $*=\varphi \varepsilon=\varepsilon \varphi$.

The following anti-involutions preserving $X, T, q, t$ will be important below, $\diamond \stackrel{\text { def }}{=} \varphi \sigma$ and $\psi \stackrel{\text { def }}{=} \diamond \tau_{+}^{-1}$ :

$$
\begin{gathered}
\pi^{\diamond}=\pi, Y^{\diamond}=T Y T^{-1}=\pi Y \pi, \quad \psi(\pi)=\widetilde{\pi} \stackrel{\text { def }}{=} \tau_{+}(\pi) \\
=q^{-1 / 4} X \pi=q^{1 / 4} \pi X^{-1}, \psi\left(Y^{-1}\right)=\widetilde{X} \stackrel{\text { def }}{=} \widetilde{\pi} T^{-1}=q^{1 / 4} Y X .
\end{gathered}
$$

1.1.2. Inner products. The polynomial representation can be supplied with inner products in various ways. The main ones are in terms of the function:

$$
\mu(X ; q, t) \stackrel{\text { def }}{=} \prod_{j=0}^{\infty} \frac{\left(1-q^{j} X^{2}\right)\left(1-q^{j+1} X^{-2}\right)}{\left(1-t q^{j} X^{2}\right)\left(1-t q^{j+1} X^{-2}\right)} .
$$

For the constant term functional

$$
\mathscr{X} \ni f \mapsto\langle f\rangle=\operatorname{ct}(f) \in \mathrm{C}_{q, t},
$$

we define three symmetric inner products in $\mathscr{X}$ :

$$
((f, g))=\left\langle f g^{*} \mu\right\rangle,\langle f, g\rangle=\langle f g \mu\rangle,\langle f, g\rangle^{\prime}=\left\langle f g \widetilde{\gamma}^{\prime} \mu\right\rangle .
$$

Here $\widetilde{\gamma}^{\prime} \stackrel{\text { def }}{=} \sum_{m=-\infty}^{\infty} q^{m x+m^{2} / 4}$ is an expansion of $q^{-x^{2}}$ in the following sense. The product $q^{x^{2}} \widetilde{\gamma}^{\prime}$ is a z/2-periodic function in terms of $x$ provided that $|q|<1$. Recall that $X=q^{x}$.

The anti-involutions of $\mathcal{H} \ni H$ corresponding to these forms are those from $(1.8,1.9)$, namely:

$$
\begin{aligned}
& ((f, H(g)))=\left(\left(H^{*}(f), g\right)\right),\langle f, H(g)\rangle=\left\langle H^{\diamond}(f), g\right\rangle, \\
& \langle f, H(g)\rangle^{\prime}=\left\langle H^{\psi}(f), g\right\rangle^{\prime} \text { for } f, g \in \mathscr{X}, H^{\psi}=\psi(H) .
\end{aligned}
$$

1.2. The E-polynomials. Let us assume that $k$ is generic; we set $t=q^{k}$. The definition of nonsymmetric polynomials is as follows:

$$
\begin{array}{r}
Y E_{n}=q^{-n_{\sharp}} E_{n} \text { for } n \in \mathrm{Z}, \\
n_{\sharp}=\left\{\begin{array}{cc}
\frac{n+k}{2} & n>0, \\
\frac{n-k}{2} & n \leq 0,
\end{array}\right\} \text {, note that } 0_{\sharp}=-\frac{k}{2} .
\end{array}
$$


The normalization is $E_{n}=X^{n}+$ "lower terms", where by "lower terms", we mean polynomials in terms of $X^{ \pm m}$ as $|m|<n$ and, additionally, $X^{|n|}$ for negative $n$. It gives a filtration in $\mathscr{X}$; check that $Y$ preserves the filtration, which justifies the definition from (1.13).

The $E_{n}(n \in \mathrm{Z})$ are called the nonsymmetric Macdonald polynomials or simply $E$-polynomials. Obviously, $E_{0}=1, E_{1}=X$.

1.2.1. The intertwiners. The first intertwiner comes from the AHA theory:

$$
\Phi \stackrel{\text { def }}{=} T+\frac{t^{1 / 2}-t^{-1 / 2}}{Y^{-2}-1}: \Phi Y=Y^{-1} \Phi .
$$

The second is $\Pi \stackrel{\text { def }}{=} q^{1 / 4} \tau_{+}(\pi)$; obviously, $\Pi^{2}=q^{1 / 2}$. Explicitly,

$$
\Pi=X \pi=q^{1 / 2} \pi X^{-1}: \Pi Y=q^{-1 / 2} Y^{-1} \Pi \text {. }
$$

Use that $\phi(\Pi)=\Pi$ to deduce the latter relation from $\Pi X \Pi^{-1}=$ $q^{1 / 2} X^{-1}$. The $\Pi$-type intertwiner is due to Knop and Sahi for $A_{n}$ (the case of arbitrary reduced systems was considered in [Ch2]). Since $\Phi, \Pi$ "intertwine" $Y^{ \pm 1}$, they can be used for generating the $E$-polynomials. Namely,

$$
\begin{aligned}
& E_{n+1}=q^{n / 2} \Pi\left(E_{-n}\right) \text { for } n \geq 0, \\
& E_{-n}=t^{1 / 2}\left(T+\frac{t^{1 / 2}-t^{-1 / 2}}{q^{2 n_{\sharp}}-1}\right) E_{n}
\end{aligned}
$$

and, beginning with $E_{0}=1$, one can readily construct the whole family of $E$-polynomials. For instance,

$$
\begin{aligned}
T(X) & =t^{1 / 2} X^{-1}+\frac{\left(t^{1 / 2}-t^{-1 / 2}\right)\left(X^{-1}-X\right)}{X^{2}-1} \\
& =t^{1 / 2} X^{-1}-\left(t^{1 / 2}-t^{-1 / 2}\right) X^{-1}=t^{-1 / 2} X^{-1}, \\
E_{-1} & =t^{1 / 2}\left(T+\frac{t^{1 / 2}-t^{-1 / 2}}{q t-1}\right) E_{1}=X^{-1}+\frac{1-t}{1-t q} X .
\end{aligned}
$$

Using $\Pi$,

$$
E_{2}=q^{1 / 2} \Pi E_{-1}=X^{2}+q \frac{1-t}{1-t q} .
$$

Applying $\Phi$ and then $\Pi$ again,

$$
\begin{aligned}
& E_{-2}=X^{-2}+\frac{1-t}{1-t q^{2}} X^{2}+\frac{(1-t)\left(1-q^{2}\right)}{\left(1-t q^{2}\right)(1-q)} \\
& E_{3}=X^{3}+q^{2} \frac{1-t}{1-t q^{2}} X^{-1}+q \frac{(1-t)\left(1-q^{2}\right)}{(1-t q)(1-q)} X .
\end{aligned}
$$


It is not difficult to find the general formula. See, e.g., (6.2.7) from [Ma] for integral $k$. However, recalculating these formulas from integral $k$ to generic $k$ is not too simple; we will provide the exact formulas for the $E$-polynomials below (in the form we need them).

The following properties of $n_{\sharp}$ reflect (1.16):

$$
(1-n)_{\sharp}=1 / 2-n_{\sharp} \text { for all } n \in \mathrm{Z},(-n)_{\sharp}=-n_{\sharp} \text { when } n \neq 0 \text {. }
$$

1.2.2. The E-Pieri rules. For any $n \in \mathrm{Z}$, we have the evaluation formula

$$
E_{n}\left(t^{-1 / 2}\right)=t^{-|n| / 2} \prod_{0<j<|\widetilde{n}|} \frac{1-q^{j} t^{2}}{1-q^{j} t},
$$

where

$$
|\widetilde{n}|=|n|+1 \text { if } n \leq 0 \text { and }|\widetilde{n}|=|n| \text { if } n>0 .
$$

It is used to introduce the nonsymmetric spherical polynomials

$$
\mathcal{E}_{n}=\frac{E_{n}}{E_{n}\left(t^{-1 / 2}\right)}
$$

This normalization is important in many constructions due to the $d u$ ality formula: $\mathcal{E}_{m}\left(q^{n_{\sharp}}\right)=\mathcal{E}_{n}\left(q^{m_{\sharp}}\right)$. The Pieri rules are the simplest for the $E$-spherical polynomials:

$$
\begin{gathered}
X \mathcal{E}_{n}=\frac{t^{-1 / 2 \pm 1} q^{-n}-t^{1 / 2}}{t^{ \pm 1} q^{-n}-1} \mathcal{E}_{n+1}+\frac{t^{1 / 2}-t^{-1 / 2}}{t^{ \pm 1} q^{-n}-1} \mathcal{E}_{1-n} \\
X^{-1} \mathcal{E}_{n}=\frac{t^{1 / 2 \pm 1} q^{-n+1}-t^{-1 / 2}}{t^{ \pm 1} q^{-n+1}-1} \mathcal{E}_{n-1}-\frac{t^{1 / 2}-t^{-1 / 2}}{t^{ \pm 1} q^{-n+1}-1} \mathcal{E}_{1-n}
\end{gathered}
$$

Here the sign is $\pm=+$ if $n \leq 0$ and $\pm=-$ if $n>0$. These formulas give an alternative approach to constructing the $E$-polynomials and establishing their connections with other theories, for instance, with the $p$-adic one.

Let us provide the norm formulas for the spherical and standard polynomials

$$
\begin{aligned}
\left\langle\mathcal{E}_{m} \mathcal{E}_{n}^{*} \mu_{\circ}\right\rangle & =\delta_{m n} \prod_{0<j<|\widetilde{n}|} \frac{1-q^{j}}{t^{-1}-q^{j} t}, \\
\left\langle E_{m} E_{n}^{*} \mu_{\circ}\right\rangle & =\delta_{m n} \prod_{0<j<|\widetilde{n}|} \frac{\left(1-q^{j}\right)\left(1-q^{j} t^{2}\right)}{\left(1-q^{j} t\right)\left(1-q^{j} t\right)}
\end{aligned}
$$

where we use $|\widetilde{n}|$ from (1.20), and $\mu_{\circ} \stackrel{\text { def }}{=} \mu /\langle\mu\rangle$. 
1.2.3. Rogers' polynomials. Let us introduce the Rogers polynomials:

$$
\begin{aligned}
P_{n} & =\left(1+t^{1 / 2} T\right)\left(E_{n}\right)=(1+s)\left(\frac{t-X^{2}}{1-X^{2}} E_{n}\right) \\
& =E_{-n}+\frac{t-t q^{n}}{1-t q^{n}} E_{n} \text { for } n \geq 0 .
\end{aligned}
$$

The leading term is $X^{n}+X^{-n}: P_{n}=X^{n}+X^{-n}+$ "lower terms". They are eigenfunctions of the following well-known operator

$$
\mathcal{L}=\frac{t^{1 / 2} X-t^{-1 / 2} X^{-1}}{X-X^{-1}} \Gamma+\frac{t^{1 / 2} X^{-1}-t^{-1 / 2} X}{X^{-1}-X} \Gamma^{-1},
$$

where we set $\Gamma(f(x))=f(x+1 / 2), \Gamma(X)=q^{1 / 2} X$, The operator $\mathcal{L}$ is the restriction of $Y+Y^{-1}$ to symmetric polynomials; this is the key point of the DAHA approach to the theory of the Macdonald polynomials.

The exact eigenvalues are as follows:

$$
\mathcal{L}\left(P_{n}\right)=\left(q^{n / 2} t^{1 / 2}+q^{-n / 2} t^{-1 / 2}\right) P_{n}, n \geq 0 .
$$

It is obvious from the latter that the $P$-polynomials are $*$-invariant. Using directly (2.6) and that $\eta(T)=T^{-1}$, for $\eta$ defined by (2.19),

$$
\begin{gathered}
P_{n}^{*}=\left(\left(1+t^{1 / 2} T\right)\left(E_{n}\right)\right)^{*}=\left(1+t^{-1 / 2} T^{-1}\right)\left(E_{n}^{*}\right)= \\
\left(1+t^{-1 / 2} T^{-1}\right)\left(t^{1 / 2} T\left(E_{n}\right)\right)=\left(1+t^{1 / 2} T\right)\left(E_{n}\right)=P_{n} .
\end{gathered}
$$

The evaluation formula reads:

$$
P_{n}\left(t^{ \pm 1 / 2}\right)=t^{-n / 2} \prod_{0 \leq j \leq n-1} \frac{1-q^{j} t^{2}}{1-q^{j} t} .
$$

The spherical $P$-polynomials $\mathcal{P}_{n} \stackrel{\text { def }}{=} P_{n} / P_{n}\left(t^{1 / 2}\right)$ satisfy the duality $\mathcal{P}_{n}\left(t^{1 / 2} q^{m / 2}\right)=\mathcal{P}_{m}\left(t^{1 / 2} q^{n / 2}\right)$. The norm formula reads:

$$
\left\langle P_{m}(X) P_{n}(X) \mu_{\circ}\right\rangle=\delta_{m n} \prod_{j=0}^{n-1} \frac{\left(1-q^{j+1}\right)\left(1-t^{2} q^{j}\right)}{\left(1-t q^{j+1}\right)\left(1-t q^{j}\right)},
$$

as $m, n \geq 0$.

1.2.4. Explicit formulas. Let us begin with the well-known formulas for the Rogers polynomials $(n \geq 0)$ :

$$
P_{n}=X^{n}+X^{-n}+\sum_{j=1}^{[n / 2]} M_{n-2 j} \prod_{i=0}^{j-1} \frac{\left(1-q^{n-i}\right)}{\left(1-q^{1+i}\right)} \frac{\left(1-t q^{i}\right)}{\left(1-t q^{n-i-1}\right)},
$$

where $M_{n}=X^{n}+X^{-n}(n>0)$ and $M_{0}=1$. 
The formulas for the $E$-polynomials are as follows $(n>0)$ :

$$
\begin{aligned}
& E_{-n}=X^{-n}+X^{n} \frac{1-t}{1-t q^{n}}+\sum_{j=1}^{[n / 2]} X^{2 j-n} \prod_{i=0}^{j-1} \frac{\left(1-q^{n-i}\right)}{\left(1-q^{1+i}\right)} \frac{\left(1-t q^{i}\right)}{\left(1-t q^{n-i}\right)} \\
& +\sum_{j=1}^{[(n-1) / 2]} X^{n-2 j} \frac{\left(1-t q^{j}\right)}{\left(1-t q^{n-j}\right)} \prod_{i=0}^{j-1} \frac{\left(1-q^{n-i}\right)}{\left(1-q^{1+i}\right)} \frac{\left(1-t q^{i}\right)}{\left(1-t q^{n-i}\right)} \\
& E_{n}=X^{n}+\sum_{j=1}^{[n / 2]} X^{2 j-n} q^{n-j} \frac{\left(1-q^{j}\right)}{\left(1-q^{n-j}\right)} \prod_{i=0}^{j-1} \frac{\left(1-q^{n-i-1}\right)}{\left(1-q^{1+i}\right)} \frac{\left(1-t q^{i}\right)}{\left(1-t q^{n-i-1}\right)} \\
& +\sum_{j=1}^{[(n-1) / 2]} X^{n-2 j} q^{j} \prod_{i=0}^{j-1} \frac{\left(1-q^{n-i-1}\right)}{\left(1-q^{1+i}\right)} \frac{\left(1-t q^{i}\right)}{\left(1-t q^{n-i-1}\right)} .
\end{aligned}
$$

\section{Global functions}

2.1. Spherical functions. We will use $n_{\sharp}=(n+\operatorname{sgn}(n) k) / 2$ for integers $n \neq 0$ and $0_{\sharp}=-k / 2$. From now on $\operatorname{sgn}(0) \stackrel{\text { def }}{=}-1$, i.e., we will always treat 0 as a negative number; cf. (1.13). As above, let $\widetilde{\gamma}^{\prime} \stackrel{\text { def }}{=} \sum_{n=-\infty}^{\infty} q^{n^{2} / 4} X^{n}, X=q^{x}$; recall that the product $q^{x^{2}} \widetilde{\gamma}^{\prime}(x)$ is a $\mathrm{Z} / 2$-periodic function of $x$. We denote the constant term functional, the coefficient of $X^{0}$, by $\langle\cdot\rangle$. We set

$$
\begin{aligned}
\mu_{\circ} \stackrel{\text { def }}{=} \mu /\langle\mu\rangle & =1+\frac{t-1}{1-q t}\left(X^{2}+q X^{-2}\right)+\ldots, \\
\text { where }\langle\mu\rangle & =\prod_{j=1}^{\infty} \frac{\left(1-t q^{j}\right)^{2}}{\left(1-t^{2} q^{j}\right)\left(1-q^{j}\right)} .
\end{aligned}
$$

Recall that

$$
X^{*}=X^{-1},\left(q^{1 / 4}\right)^{*}=q^{-1 / 4},\left(t^{1 / 2}\right)^{*}=t^{-1 / 2} .
$$

The series $\mu_{\circ}$ is $*$-invariant.

2.1.1. Gauss-type integrals. For arbitrary $m, n \in \mathrm{Z}$,

$$
\begin{aligned}
& \left\langle\mathcal{E}_{n} \mathcal{E}_{m} \widetilde{\gamma}^{\prime} \mu_{\circ}\right\rangle=q^{\frac{m^{2}+n^{2}+2 k(|m|+|n|)}{4}} \mathcal{E}_{m}\left(q^{n_{\sharp}}\right)\left\langle\widetilde{\gamma}^{\prime} \mu_{\circ}\right\rangle, \\
& \left\langle\mathcal{E}_{n} \mathcal{E}_{m}^{*} \widetilde{\gamma}^{\prime} \mu_{\circ}\right\rangle=q^{\frac{m^{2}+n^{2}+2 k(|m|+|n|)}{4}} \mathcal{E}_{m}^{*}\left(q^{n_{\sharp}}\right)\left\langle\widetilde{\gamma}^{\prime} \mu_{\circ}\right\rangle .
\end{aligned}
$$


In these formulas,

$$
\left\langle\widetilde{\gamma}^{\prime} \mu_{\circ}\right\rangle=\prod_{j=1}^{\infty} \frac{1-q^{j}}{1-t q^{j}},\left\langle\widetilde{\gamma}^{\prime} \mu\right\rangle=\prod_{j=1}^{\infty} \frac{1-t q^{j}}{1-t^{2} q^{j}} .
$$

See [Ch1], Theorem 2.7.1.

The $*$-conjugations of the $\mathcal{E}$-polynomials are as follows:

$$
\begin{aligned}
& \mathcal{E}_{m}^{*}=t^{-\frac{1}{2}} T\left(\mathcal{E}_{m}\right)=q^{-(m+(1+\operatorname{sgn}(m)) k) / 2} \pi\left(\mathcal{E}_{m}\right)=t^{-\frac{1}{2}} X^{-1} \mathcal{E}_{1-m}, \\
& \text { where }(m+(1+\operatorname{sgn}(m)) k) / 2=m_{\sharp}+k / 2 .
\end{aligned}
$$

where $m \in \mathrm{Z}, \operatorname{sgn}(0)=-1$. See, e.g., [Ch1], Proposition 2.5.13.

The conjugation is somewhat simpler in terms of the $E$-polynomials:

$$
E_{m}^{*}=t^{\operatorname{sgn}(m) / 2} T\left(E_{m}\right)=q^{-\frac{m}{2}} \pi\left(E_{m}\right)=X^{-1} E_{1-m} \text { for } m \in \mathrm{z} .
$$

Using (1.19), we obtain:

$$
\begin{aligned}
& \left\langle E_{n} E_{m} \widetilde{\gamma}^{\prime} \mu_{\circ}\right\rangle=\prod_{j=1}^{|\widetilde{n}|-1} \frac{1-q^{j} t^{2}}{1-q^{j} t} \prod_{j=1}^{\infty} \frac{\left(1-q^{j}\right)}{\left(1-q^{j} t\right)} q^{\frac{m^{2}+n^{2}+2 k|m|}{4}} E_{m}\left(q^{n_{\sharp}}\right), \\
& \left\langle E_{n} E_{m}^{*} \widetilde{\gamma}^{\prime} \mu_{\circ}\right\rangle=\prod_{j=1}^{|\widetilde{n}|-1} \frac{1-q^{j} t^{2}}{1-q^{j} t} \prod_{j=1}^{\infty} \frac{\left(1-q^{j}\right)}{\left(1-q^{j} t\right)} q^{\frac{m^{2}+n^{2}+2 k|m|}{4}} E_{m}^{*}\left(q^{n_{\sharp}}\right) .
\end{aligned}
$$

Switching to $\mu$, the formulas from $(2.2,2.3)$ read:

$$
\begin{aligned}
& \left\langle E_{n} E_{m} \widetilde{\gamma}^{\prime} \mu\right\rangle=\prod_{j=|\widetilde{n}|}^{\infty} \frac{1-q^{j} t}{1-q^{j} t^{2}} q^{\frac{m^{2}+n^{2}+2 k|m|}{4}} E_{m}\left(q^{n_{\sharp}}\right), \\
& \left\langle E_{n} E_{m}^{*} \widetilde{\gamma}^{\prime} \mu\right\rangle=\prod_{j=|\widetilde{n}|}^{\infty} \frac{1-q^{j} t}{1-q^{j} t^{2}} q^{\frac{m^{2}+n^{2}+2 k|m|}{4}} E_{m}^{*}\left(q^{n_{\sharp}}\right) .
\end{aligned}
$$

Use the second formula from (2.4).

2.1.2. Fourier transforms. The formulas for the Gauss integrals can be represented algebraically, up to a (global) coefficient of proportionality, as follows. In the theorem below, $q^{ \pm x^{2}}$ will be the formal Gaussian satisfying the defining relations from (1.2):

$$
\begin{gathered}
s\left(q^{ \pm x^{2}}\right)=q^{ \pm x^{2}}, \omega\left(q^{ \pm x^{2}}\right)=q^{ \pm 1 / 4} X^{\mp 1} q^{ \pm x^{2}}, \\
\text { where } s(x)=-x, \omega(f(x))=f(x-1 / 2), \\
Y\left(q^{ \pm x^{2}}\right)=\omega\left(q^{ \pm x^{2}}\right)=q^{ \pm 1 / 4} X^{\mp 1} q^{ \pm x^{2}}, \\
\text { where } \pi=\omega s, \pi(f(x))=f(1 / 2-x) .
\end{gathered}
$$

For instance, one can take $\widetilde{\gamma}^{\prime}$ here as $q^{-x^{2}}$. 
Theorem 2.1. (i) The $\mathrm{C}\left(q^{1 / 4}, t^{1 / 2}\right)$-linear map defined by

$$
\mathbf{F}_{\sigma}: \mathcal{X}_{q^{-x^{2}}}^{\ni} E_{m} q^{-x^{2}} \mapsto q^{\frac{m^{2}+2 k|m|}{4}} E_{m} q^{+x^{2}} \in \mathcal{X} q^{+x^{2}},
$$

where $m \in \mathrm{Z}$, induces the automorphism $\sigma$ on the algebra $\mathcal{H}$ naturally acting in $\mathcal{X} q^{\mp x^{2}}$. Equivalently, $\sigma=\varphi \diamond$.

(ii) Let

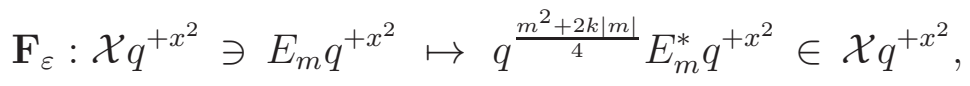

where $q^{1 / 4} \mapsto q^{-1 / 4}$ and $t^{1 / 2} \mapsto t^{-1 / 2}$, i.e., the constants are $*$-conjugated under the action of $\mathbf{F}_{\varepsilon}$. This map induces the involution $\varepsilon$ on $\mathcal{H}$; equivalently, $\varepsilon=\varphi *$.

(iii) Formally conjugating (2.8), let

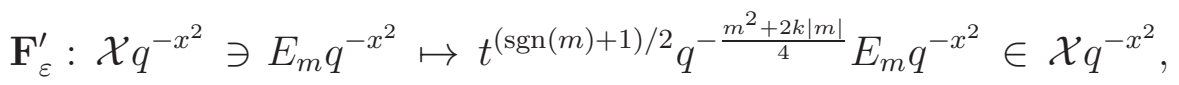

where $q^{1 / 4} \mapsto q^{-1 / 4}$ and $t^{1 / 4} \mapsto t^{-1 / 4}$. This map induces the same involution $\varepsilon$ on $\mathcal{H}$.

Here we closely follow [Ch3], Theorem 5.1. We will clarify the way to calculate the corresponding DAHA isomorphisms in the next section.

Note that the (formal) conjugation of $(i)$ eventually leads to the transform equivalent to that from (iii). Use (2.5) and the first relation from (1.18).

2.1.3. Reproducing kernels. The general fact is that if the map $f \mapsto$ $\widehat{f}(m)=\left\langle\mathcal{E}_{m}, f\right\rangle_{\alpha}$ can be naturally extended to a morphism $\mathbf{F}$ of $\mathcal{H}-$ modules, then it corresponds to the automorphism $\beta=\varphi \alpha$, where $\varphi$ is from (1.3), $\alpha$ is the anti-involution of $\mathcal{H}$ associated to $\langle\cdot, \cdot\rangle_{\alpha}$. The latter is assumed to be nondegenerate symmetric inner product or an anti-symmetric one (for the second component). Here $f$ may belong to various function spaces, including $\mathscr{X} q^{l x^{2}}(l \in Z)$, their completions and their delta-counterparts.

The fundamental concept of Fourier analysis (and representation theory) is the reproducing kernel. Given a transform $\mathbf{B}$, it is defined as follows:

$$
G(X, \Lambda)=\sum_{n} f_{n}^{\prime}(X) \mathbf{B}\left(f_{n}\right)(\Lambda), \text { where }\left\langle f_{m}^{\prime}, f_{n}\right\rangle_{\alpha}=\delta_{m n}
$$

The basis $\left\{f_{n}\right\}$ in the initial function space can be arbitrary; $\left\{f_{n}^{\prime}\right\}$ is its dual. Provided the existence, we assume that $\mathbf{B}$ induces the 
isomorphism $\beta$ for the operators. Then

$$
\langle G(X, \Lambda), f(X)\rangle_{\alpha}=\mathbf{B}(f)(\Lambda), H(G)=\widehat{\beta \alpha(H)}(G)
$$

for $H \in \mathcal{H}$, where we consider $f_{n}$ and $f_{n}^{\prime}$ as functions of $X$ and suppose that $\mathbf{B}$ sends them to functions of $\Lambda$, i.e., $\mathbf{B}: f(X) \mapsto \widehat{f}(\Lambda)$. By $H, \widehat{H}$, we mean this operator acting in the domain and range of $\mathbf{B}$, i.e., on functions of $X$ and $\Lambda$ respectively. For instance, $Y(G(X, \Lambda))=$ $\Lambda^{-1} G(X, \Lambda)$.

The formal independence of $G$ of the choice of the basis $\left\{f_{n}\right\}$ becomes a non-trivial issue in the functional analysis. The better the basis (and the space), the better analytic theory of $G$. In the $q$-theory, the best choice is the basis of the $E$-polynomials multiplied by the Gaussian.

2.1.4. Nonsymmetric global functions. Theorem 2.1 results in the following two formulas (both are from [Ch3]). Using $(i)$ with the pairing $\left\langle f g \mu_{\circ}\right\rangle$ for

$$
f_{n}=E_{n} q^{-x^{2}} \text { and } f_{n}^{\prime}=E_{n}^{*} q^{x^{2}} /\left\langle E_{n} E_{n}^{*} \mu_{\circ}\right\rangle
$$

and $(i i)$ with pairing $\left\langle f g^{*} \mu_{\circ}\right\rangle$ for

$$
f_{n}=E_{n} q^{x^{2}} \text { and } f_{n}^{\prime}=E_{n} q^{x^{2}} /\left\langle E_{n} E_{n}^{*} \mu_{\circ}\right\rangle,
$$

we arrive at the following coinciding formulas:

$$
\begin{aligned}
\frac{\widetilde{\gamma}^{\prime}(X) \widetilde{\gamma}^{\prime}(\Lambda)}{\widetilde{\gamma}^{\prime}\left(t^{1 / 2}\right)} G(X ; \Lambda) & =\sum_{n=-\infty}^{\infty} q^{\frac{|n|^{2}}{4}} t^{\frac{|n|}{2}} \frac{E_{n}^{*}(X) E_{n}(\Lambda)}{\left\langle E_{n} E_{n}^{*} \mu_{\circ}\right\rangle} \\
& =\sum_{n=-\infty}^{\infty} q^{\frac{|n|^{2}}{4}} t^{\frac{|n|}{2}} \frac{E_{n}(X) E_{n}^{*}(\Lambda)}{\left\langle E_{n} E_{n}^{*} \mu_{\circ}\right\rangle}
\end{aligned}
$$

where we use $\widetilde{\gamma}^{\prime}$ for $q^{-x^{2}}$. The inner products $\left\langle E_{n} E_{n}^{*} \mu_{\circ}\right\rangle$ in these formulas and in those below are provided in (1.24).

It is assumed here that $|q|<1 ; X, \Lambda$ are arbitrary apart from the zeros of $\widetilde{\gamma}^{\prime}(X) \widetilde{\gamma}^{\prime}(\Lambda)$. The formula converges for any $q$ not equal to a root of unity and is meromorphic. When $|q|=1$, the analyticity is understood with respect to the directions not tangent to the unit circle ( $q$ must not be a root of unity).

Checking the coincidence of these expressions for $G$ is not difficult because they satisfy the same relations from (2.12). The basic ones are:

$$
Y(G)=\Lambda^{-1} G, X^{-1} G=Y_{\Lambda}(G), T(G)=T_{\Lambda}(G),
$$

where the operators indexed by $\Lambda$ act in terms of $\Lambda$. One can check this coincidence directly. Substitute $E_{m}^{*}=t^{\operatorname{sgn}(m) / 2} T\left(E_{m}\right)$ and use that 
$T(G)=T_{\Lambda}(G)$; equivalently, the relation $E_{m}^{*}=X^{-1} E_{1-m}$ can be used.

Similarly, the formulas from (iii) coupled with the inner product $\left\langle f g^{*} \mu_{\circ}\right\rangle$ and the basic elements

$$
f_{n}=E_{n} q^{-x^{2}}, n \in \mathrm{Z}, f_{n}^{\prime}=E_{n} q^{-x^{2}} /\left\langle E_{n} E_{n}^{*} \mu_{\circ}\right\rangle
$$

result in the second function:

$$
\begin{aligned}
& \frac{\widetilde{\gamma}(X) \widetilde{\gamma}(\Lambda)}{\widetilde{\gamma}\left(t^{1 / 2}\right)} G^{\checkmark}(X ; \Lambda) \\
& =\sum_{n=-\infty}^{\infty} q^{-\frac{|n|^{2}}{4}} t^{-\frac{|n|}{2}} t^{(\operatorname{sgn}(n)+1) / 2} \frac{E_{n}(X) E_{n}(\Lambda)}{\left\langle E_{n} E_{n}^{*} \mu_{\circ}\right\rangle}
\end{aligned}
$$

where $|q|>1$ and $\widetilde{\gamma} \stackrel{\text { def }}{=} \sum_{n=-\infty}^{\infty} q^{-n^{2} / 4} X^{n}$.

It satisfies the relations from (2.12) and (2.14):

$$
Y\left(G^{\checkmark}\right)=\Lambda^{-1} G^{\checkmark}, X^{-1} G^{\checkmark}=\widehat{Y}\left(G^{\checkmark}\right), T\left(G^{\checkmark}\right)=\widehat{T}\left(G^{\checkmark}\right),
$$

i.e., the same relations as for $G$.

The functions $G, G^{\checkmark}$ are called the global nonsymmetric spherical functions.

The symmetric (even) global functions read as follows:

$$
\begin{aligned}
& \frac{\widetilde{\gamma}^{\prime}(X) \widetilde{\gamma}^{\prime}(\Lambda)}{\widetilde{\gamma}^{\prime}\left(t^{1 / 2}\right)} F(X ; \Lambda)=\sum_{n=0}^{\infty} q^{\frac{n^{2}}{4}} t^{\frac{n}{2}} \frac{P_{n}(X) P_{n}(\Lambda)}{\left\langle P_{n} P_{n} \mu_{\circ}\right\rangle}, \text { for }|q|<1 \\
& \frac{\widetilde{\gamma}(X) \widetilde{\gamma}(\Lambda)}{\widetilde{\gamma}\left(t^{1 / 2}\right)} F^{\checkmark}(X ; \Lambda)=\sum_{n=0}^{\infty} q^{-\frac{n^{2}}{4}} t^{-\frac{n}{2}} \frac{P_{n}(X) P_{n}(\Lambda)}{\left\langle P_{n} P_{n} \mu_{\circ}\right\rangle},|q|>1
\end{aligned}
$$

Both satisfy the same difference equation $\mathcal{L}(F)=\left(\Lambda+\Lambda^{-1}\right) F$ for $\mathcal{L}$ from (1.26). The second function is obtained from the first upon the formal star-conjugation

$$
q \mapsto q^{-1}, t \mapsto t^{-1}, X \mapsto X^{-1}, \Lambda \mapsto \Lambda^{-1},
$$

preserving $\mathcal{L}$ ( $\Gamma$ remains fixed).

The action of $*$-conjugation on the $G$-functions is more involved.

Theorem 2.2. Let $\widetilde{G}=\left(G^{\checkmark}\right)^{*}$ be the result of the formal conjugation of $q, t$ and $X, \Lambda$. Namely, we set $t=q^{k}, X=q^{x}, \Lambda=q^{\lambda}$ and replace $q$ by $q^{-1}$ in the formula for $G^{\checkmark}$ without changing $k, x, \lambda$; for instance, $\widetilde{\gamma}^{*}=\widetilde{\gamma}^{\prime}$. The convergence of the series for $\widetilde{G}$ is for $|q|<1$ and

$$
\left(t^{1 / 2} T^{-1}\right)(\widetilde{G}(X, \Lambda))=G(X, \Lambda)=t^{1 / 2} \Lambda \pi(\widetilde{G}(X, \Lambda)) .
$$


Proof. The conjugation of functions results in the application of the automorphism $\eta$ at level of operators (not $*$ as one may expect). See [Ch1]:

$$
\begin{gathered}
\eta: T \mapsto T^{-1}, \pi \mapsto \pi, X \mapsto X^{-1}, q^{1 / 4} \mapsto q^{-1 / 4}, t^{1 / 2} \mapsto t^{-1 / 2} \\
H\left(f^{*}\right)=(\eta(H)(f))^{*} \text { for } f \in \mathscr{X}=\mathrm{C}_{q, t}\left[X^{ \pm 1}\right], H \in \mathcal{H} .
\end{gathered}
$$

It must be done in the $X$-space and in the $\Lambda$-space. For instance, the eigenvalue problem $Y\left(G^{\checkmark}\right)=\Lambda^{-1} G^{\checkmark}$ becomes $\eta(Y)(\widetilde{G})=\Lambda \widetilde{G}$. Let us calculate the conjugations of the relations from (2.14).

Since $\eta(Y)=T Y^{-1} T^{-1}$, we obtain:

$$
\begin{gathered}
T(\widetilde{G})=T_{\Lambda}(\widetilde{G}), \eta(Y)(\widetilde{G})=\Lambda \widetilde{G}, X \widetilde{G}=\eta(Y)_{\Lambda}(G), \\
T Y^{-1} T^{-1}(\widetilde{G})=\Lambda \widetilde{G}=\Lambda T T_{\Lambda}^{-1} \widetilde{G} \Rightarrow Y^{-1}\left(T^{-1}(\widetilde{G})\right)=\Lambda T_{\Lambda}^{-1}(\widetilde{G}), \\
X \widetilde{G}=\left(T Y^{-1} T^{-1}\right)_{\Lambda}(\widetilde{G}) \Rightarrow X \widetilde{G}=\left(T_{\Lambda} Y_{\Lambda}^{-1} T_{\Lambda}^{-1} T_{\Lambda} T^{-1}\right)(\widetilde{G}) \\
=T^{-1}\left(T_{\Lambda} Y_{\Lambda}^{-1}\right)(\widetilde{G}) \Rightarrow X^{-1}\left(T^{-1}(\widetilde{G})\right)=Y_{\Lambda}\left(T_{\Lambda}^{-1}(\widetilde{G})\right)=Y_{\Lambda}\left(T^{-1}(\widetilde{G})\right) .
\end{gathered}
$$

Therefore $T^{-1}(\widetilde{G})$ satisfies all relations from (2.14) and must coincide with $G$ up to normalization. The normalization factor can be readily determined. As a matter of fact, this general justification is not necessary at all (for $A_{1}$ ) because of the following explicit identification of the corresponding series:

$$
\begin{aligned}
& \left(\sum_{n=-\infty}^{\infty} q^{-\frac{|n|^{2}}{4}} t^{-\frac{|n|}{2}} t^{+(\operatorname{sgn}(n)+1) / 2} \frac{E_{n}(X) E_{n}(\Lambda)}{\left\langle E_{n} E_{n}^{*} \mu_{\circ}\right\rangle}\right)^{*} \\
= & \sum_{n=-\infty}^{\infty} q^{+\frac{|n|^{2}}{4}} t^{+\frac{|n|}{2}} t^{-(\operatorname{sgn}(n)+1) / 2} \frac{E_{n}^{*}(X) E_{n}^{*}(\Lambda)}{\left\langle E_{n} E_{n}^{*} \mu_{\circ}\right\rangle} \\
= & \sum_{n=-\infty}^{\infty} q^{+\frac{|n|^{2}}{4}} t^{+\frac{|n|}{2}} t^{-(\operatorname{sgn}(n)+1) / 2} \frac{t^{\operatorname{sgn}(n) / 2} T\left(E_{n}(X)\right) E_{n}^{*}(\Lambda)}{\left\langle E_{n} E_{n}^{*} \mu_{\circ}\right\rangle} .
\end{aligned}
$$

The second equality in (2.18) follows from the first:

$$
t^{1 / 2} \pi(\widetilde{G})=t^{1 / 2}\left(Y T^{-1}\right)(\widetilde{G})=Y\left(t^{1 / 2} T^{-1}(\widetilde{G})\right)=\Lambda^{-1} G .
$$

We see that the functions $G$ and $G^{\checkmark}$ are conceptually connected through the formal conjugation, however, a certain twist is needed. It is a general fact (which was not observed in [Ch3]).

2.2. Whittaker functions. In this section, $t=q^{k}$ and $|q|<1$ unless stated otherwise. We will use the elementary difference operator $\Gamma(X)=q^{1 / 2} X$ and also $\Gamma_{k}(X) \stackrel{\text { def }}{=} q^{k / 2} X$, 
2.2.1. Whittaker limit. Etingof states in [Et] following Ruijsenaars [Rui] that

$$
\lim _{k \rightarrow-\infty} q^{-k x} \Gamma_{k} \mathcal{L} \Gamma_{-k} q^{k x}
$$

becomes the so-called $q$-Toda (difference) operator. To be exact, they considered the case of $A_{n}$.

Following [Ch5], the basic limiting procedure will be when $k$ approaches $\infty$ for $|q|<1(|t| \rightarrow 0)$, unless stated otherwise. Let

$$
æ(\mathcal{L}) \stackrel{\text { def }}{=} q^{k x} \Gamma_{k}^{-1} \mathcal{L} \Gamma_{k} q^{-k x}, \mathcal{T} \stackrel{\text { def }}{=} R \mathbb{E}(\mathcal{L})=\lim _{k \rightarrow \infty} æ(\mathcal{L}),
$$

where the second limit is the Ruijsenaars-Etingof procedure. At level of functions $F(X)$ :

$$
R \mathbb{E}(F)=\lim _{k \rightarrow \infty} q^{k x} F\left(q^{-k / 2} X\right)=\lim _{k \rightarrow \infty} q^{k x} \Gamma_{k}^{-1}(F) .
$$

Later, the case $|q|>1(|t| \rightarrow \infty)$ will be needed too. Then

$$
\begin{gathered}
æ^{\checkmark}(\mathcal{L}) \stackrel{\text { def }}{=} q^{-k x} \Gamma_{k}^{-1} \mathcal{L} \Gamma_{k} q^{k x}, \mathcal{T}^{\checkmark} \stackrel{\text { def }}{=} R E^{\checkmark}(\mathcal{L})=\lim _{k \rightarrow \infty} æ^{\checkmark}(\mathcal{L}), \\
R E^{\sqrt{ }}(F)=\lim _{k \rightarrow \infty} q^{-k x} F\left(q^{-k / 2} X\right)=\lim _{k \rightarrow \infty} q^{-k x} \Gamma_{k}^{-1}(F) .
\end{gathered}
$$

Generally, the $R E$ procedures require very specific functions $F$ to be well defined. Formally, if $\mathcal{L}(\Phi)=\left(\Lambda+\Lambda^{-1}\right) \Phi$, then

$$
\begin{aligned}
& R E(\mathcal{L})(\mathcal{W})=\left(\Lambda+\Lambda^{-1}\right) \mathcal{W} \text { for } \mathcal{W}=R \mathbb{E}(\Phi) \\
& R E^{\checkmark}(\mathcal{L})\left(\mathcal{W}^{\checkmark}\right)=\left(\Lambda+\Lambda^{-1}\right) \mathcal{W}^{\checkmark} \text { for } \mathcal{W}^{\checkmark}=R E^{\checkmark}(\Phi), \\
& \text { provided the existence of } \mathcal{W}, \mathcal{W}^{\checkmark} .
\end{aligned}
$$

At level of operators,

$$
\begin{aligned}
æ(\mathcal{L}) & =\frac{X-X^{-1}}{t^{-1 / 2} X-t^{1 / 2} X^{-1}} t^{-1 / 2} \Gamma+\frac{t X^{-1}-t^{-1} X}{t^{1 / 2} X^{-1}-t^{-1 / 2} X} t^{1 / 2} \Gamma^{-1} \\
& =\frac{X-X^{-1}}{X-t X^{-1}} \Gamma+\frac{t^{2} X^{-1}-X}{t X^{-1}-X} \Gamma^{-1}
\end{aligned}
$$

Therefore when $|t| \rightarrow 0$,

$$
\mathcal{T}=R E(\mathcal{L})=\frac{X-X^{-1}}{X} \Gamma+\Gamma^{-1}=\left(1-X^{-2}\right) \Gamma+\Gamma^{-1} .
$$

Similarly,

$$
\begin{aligned}
æ^{\checkmark}(\mathcal{L}) & =\frac{X-X^{-1}}{t^{-1 / 2} X-t^{1 / 2} X^{-1}} t^{1 / 2} \Gamma+\frac{t X^{-1}-t^{-1} X}{t^{1 / 2} X^{-1}-t^{-1 / 2} X} t^{-1 / 2} \Gamma^{-1} \\
& =\frac{X-X^{-1}}{X-t X^{-1}} \Gamma+\frac{t^{2} X^{-1}-X}{t X^{-1}-X} \Gamma^{-1}
\end{aligned}
$$


In this case $|q|>1$, so $|t| \rightarrow \infty$ and

$$
\mathcal{T}^{\checkmark}=R E^{\checkmark}(\mathcal{L})=\frac{X-X^{-1}}{-X^{-1}} \Gamma+\Gamma^{-1}=\left(1-X^{2}\right) \Gamma+\Gamma^{-1} .
$$

One of the main results of [Ch5] is the formula for the $R E$-limits of the global symmetric $q, t$-spherical function (for arbitrary reduced root systems; see the definitions there). In the $A_{1}$-case, the limit of $F(X ; \Lambda)$ from $(2.16)$ is as follows:

$$
\begin{gathered}
\mathcal{W}(X, \Lambda)= \\
\left(\widetilde{\gamma}^{\prime}(X) \widetilde{\gamma}^{\prime}(\Lambda)\right)^{-1} \sum_{m=0}^{\infty} q^{m^{2} / 4} \bar{P}_{m}(\Lambda ; q) X^{m} \prod_{s=1}^{m} \frac{1}{1-q^{s}},
\end{gathered}
$$

where $|q|<1, \prod_{s=1}^{0}=1, \Lambda=q^{\lambda}$ as for $X, \bar{P}_{m}$ are the symmetric $q$-Hermite polynomials, to be discussed next. It satisfies, among its other properties, the relation $\mathcal{T}(\mathcal{W})=\left(\Lambda+\Lambda^{-1}\right) \mathcal{W}$.

Recall that $\widetilde{\gamma}^{\prime}(X) \widetilde{\gamma}^{\prime}(\Lambda)$ divided by $q^{-x^{2}-\lambda^{2}}$ is a $\mathrm{Z} / 2$-periodic function of $X$ and of $\Lambda$. Therefore, as far as the Toda eigenvalue problem and other symmetries are concerned, $\left(\widetilde{\gamma}^{\prime}(X) \widetilde{\gamma}^{\prime}(\Lambda)\right)^{-1}$ can be replaced by $q^{x^{2}+\lambda^{2}}$. In this paper, we prefer to make all functions in terms of Laurent variables, $X$ and $\Lambda$; this explains our choice to use $\widetilde{\gamma}^{\prime}$ rather than $q^{-x^{2}}$.

We note that the series from (2.27) was introduced and discussed in [Sus] (for $A_{1}$ only), however, without the Gaussians and without any reference to the Whittaker theory. It appeared there as a quadratic exponential function and as a quadratic generating function for onedimensional $q$-Hermite polynomials.

When $|q|>1$, the $R E^{\checkmark}$-procedure results in

$$
\begin{gathered}
\mathcal{W}^{\checkmark}(X, \Lambda)= \\
(\widetilde{\gamma}(X) \widetilde{\gamma}(\Lambda))^{-1} \sum_{m=0}^{\infty} q^{-\frac{m^{2}}{4}} \bar{P}_{m}\left(\Lambda ; q^{-1}\right) X^{-m} \prod_{s=1}^{m} \frac{1}{1-q^{-s}},
\end{gathered}
$$

where $\widetilde{\gamma}$ is obtained from $\widetilde{\gamma}^{\prime}$ by the formal substitution $q \mapsto q^{-1}$.

The function $\mathcal{W}^{\checkmark}(X, \Lambda)$ is obviously $\mathcal{W}(X, \Lambda)$ upon the action of the automorphism of $\mathrm{C}\left[q^{ \pm 1 / 4}\right]\left[X^{ \pm 1}\right]$ defined by $X^{\checkmark}=X^{-1}$, and $\left(q^{1 / 4}\right)^{\checkmark}=$ $q^{-1 / 4}$. Since $x^{\checkmark}=x$ due to $X=q^{x}$, the image $\Gamma^{\checkmark}$ of $\Gamma$, sending $x \mapsto x+1 / 2$, coincides with $\Gamma$. Thus $\mathcal{T}^{\checkmark}\left(\mathcal{W}^{\checkmark}\right)=\left(\Lambda+\Lambda^{-1}\right) \mathcal{W}^{\checkmark}$, as it is supposed to be.

Note that the formulas above are valid for any $|q| \neq 1$ upon a straight algebraic transformation from $q$ to $q^{-1}$. Moreover, the analytic continuation to $|q|=1$ is possible for almost all such $q$ (roots of unity must be excluded, but not only them); see below. 
2.2.2. Harish-Chandra expansion. A systematic analytic theory of the global functions will be a subject of further papers; however, the following introduction to the Harish-Chandra $q$, $t$-theory seems quite relevant here. The corresponding nonsymmetric theory will be presented in a continuation of this paper. It is actually the best way to proceed for arbitrary root systems; for $A_{n}$, the constructions in the symmetric setting are relatively straightforward.

For $|q|<1$ and $|X|>|q|^{1 / 2}$ the asymptotic expansions and the corresponding Harish-Chandra decomposition read in the $q$-Whittaker case as follows:

$$
\begin{aligned}
& \widetilde{\gamma}^{\prime}(X) \widetilde{\gamma}^{\prime}(\Lambda) \mathcal{W}(X, \Lambda) \\
& =\langle\bar{\mu}\rangle \bar{\sigma}\left(\Lambda^{-1}\right) \widetilde{\gamma}^{\prime}(X \Lambda) \sum_{j=0}^{\infty} q^{j} X^{-2 j} \prod_{s=1}^{j} \frac{1}{\left(1-q^{s}\right)\left(1-q^{s} \Lambda^{2}\right)} \\
& +\langle\bar{\mu}\rangle \bar{\sigma}(\Lambda) \widetilde{\gamma}^{\prime}\left(X \Lambda^{-1}\right) \sum_{j=0}^{\infty} q^{j} X^{-2 j} \prod_{s=1}^{j} \frac{1}{\left(1-q^{s}\right)\left(1-q^{s} \Lambda^{-2}\right)} \\
& \text { where } \bar{\sigma}(\Lambda)=\prod_{j=0}^{\infty}\left(1-q^{j} \Lambda^{2}\right)^{-1},\langle\bar{\mu}\rangle=\prod_{j=1}^{\infty}\left(1-q^{j}\right)^{-1}
\end{aligned}
$$

are the $q$-Whittaker version of the Harish-Chandra $c$-function and the constant term of $\bar{\mu}$ from (2.40) below. This identity follows from the Whittaker part of the paper [Ch5]. The corresponding expansions exist for arbitrary (reduced) root systems (in the twisted case), but the explicit formulas for the coefficients can be involved.

Using that the terms in the right-hand solve the $q$-Toda eigenvalue problem, they can be meromorphically continued to all values of $X$. The difference equation in terms of $\Lambda$ is also known (see [Ch5]), so the right-hand side can be extended meromorphically to all $X, \Lambda$.

One can expect to exclude $q, \Lambda$ such that $q^{s} \Lambda^{ \pm 2} \neq 1$ for any $s \in \mathrm{N}$, but the poles from the both terms in the right-hand side will cancel each other at these points. Thus it is not necessary. Generally speaking (for arbitrary root systems), the cancelation of the poles in the HarishChandra decomposition formulas is difficult to check directly. The existence of the global function makes it immediate.

The inequalities $|X|>\left|q^{1 / 2}\right|$ guarantee the absolute convergence of the right-hand side (the expansions) for $|q|<1$. However, they are not needed for the left-hand side (the global function), which is analytic for any $X, \Lambda$ when $|q|<1$ or when $|q|>1$. 
Note that the convergence of the summations in the right-hand side of (2.29) becomes significantly better for $|q|>1 ; X, \Lambda$ can be arbitrary for such $q$ (as in the left-hand side). The same essentially holds for the multipliers $\langle\bar{\mu}\rangle \bar{\sigma}\left(\Lambda^{ \pm 1}\right)$. Indeed, they can be redefined in terms of the $q$-exponential series; then similar arguments can be applied.

The left-hand (global) side of (2.29) can be extended even to almost all points at the unit circle:

$$
\begin{aligned}
& |q|=1 \text { provided }\left|X \Lambda^{ \pm 1}\right|>1 / R(q), \\
& \text { where } R(q) \stackrel{\text { def }}{=} \liminf _{m \rightarrow \infty}\left|1-q^{m}\right|^{1 / m} .
\end{aligned}
$$

Here we must of course avoid the roots of unity, where $R(q)=0$, but the essence of this approach is that it is not sufficient; a greater set of points must be avoided. See, e.g., [Lub], especially, (1.17)-(1.19) there. The theory of such analytic continuations to the unit circle is classical, due to Hardy-Littlewood and others.

The analytic continuation to the unit circle (at almost all points) is, generally, only with respect to the non-tangent directions. However, the following sequences $\left\{q_{n}\right\}$ tending to $q$ are allowed. If $\left|q_{n}\right|=1$ then we must assume that $\left|1-q_{n}^{m}\right|^{1 / m}>\epsilon$ for an arbitrarily small $\epsilon>0$, almost all $m>0$ and for sufficiently large $n$.

Comment. The right-hand side of (2.29) is a weighted sum of the asymptotic series as $|X| \rightarrow \infty$ for the $q$-Toda eigenvalue problem and that for $\Lambda \mapsto \Lambda^{-1}$. This series solves the Toda eigenvalue problem and its coefficients can be uniquely determined from it. Recall that the exact convergence condition for it is $|X|>|q|^{1 / 2}$ for $|q|<1$, which becomes much better (any $X$ ) upon adding its $\Lambda^{-1}$-counterpart.

This series is an instance of the formula from [GiL] (the $G L(n)$-case), representing a certain generating function in the quantum $K$-theory of flag varieties. See the Appendix, Section 7. We note that only the asymptotic series alone appears in [GiL], not the weighted summation from (2.29).

Thus the Harish-Chandra formula (2.29) for the global Whittaker function connects that Givental-Lee theory with the algebraic geometry of affine Schubert varieties (encoded in the level one affine Demazure character formulas). The limit $q \rightarrow 1$ of (2.29) coincides with the corresponding $c$-weighted summation formula for the classical (real) Whittaker function (see [GW],[Wa]); generally, the summation is over the Weyl group. 
The spherical $q, t$-generalization of (2.29) is actually simpler to establish. The following theorem is not too difficult to justify in the rank one case. Generally, the nonsymmetric theory helps significantly.

Theorem 2.3. For the function $F(X ; \Lambda)$ from (2.16), let us assume that $|q|<1$ and $|X|<|t|^{1 / 2}|q|^{-1 / 2}$. Then

$$
\frac{\widetilde{\gamma}^{\prime}(X) \widetilde{\gamma}^{\prime}(\Lambda)}{\widetilde{\gamma}^{\prime}\left(t^{1 / 2}\right)} F(X, \Lambda)
$$

$$
\begin{gathered}
=\langle\mu\rangle \sigma(\Lambda) \widetilde{\gamma}^{\prime}\left(X \Lambda t^{-1 / 2}\right) \sum_{j=0}^{\infty}\left(\frac{q}{t}\right)^{j} X^{2 j} \prod_{s=1}^{j} \frac{\left(1-t q^{s-1}\right)\left(1-q^{s-1} t \Lambda^{-2}\right)}{\left(1-q^{s}\right)\left(1-q^{s} \Lambda^{-2}\right)} \\
+\langle\mu\rangle \sigma\left(\Lambda^{-1}\right) \widetilde{\gamma}^{\prime}\left(X \Lambda^{-1} t^{-1 / 2}\right) \sum_{j=0}^{\infty}\left(\frac{q}{t}\right)^{j} X^{2 j} \prod_{s=1}^{j} \frac{\left(1-t q^{s-1}\right)\left(1-q^{s-1} t \Lambda^{2}\right)}{\left(1-q^{s}\right)\left(1-q^{s} \Lambda^{2}\right)}
\end{gathered}
$$

where $\sigma(\Lambda)=\prod_{j=0}^{\infty} \frac{1-t q^{j} \Lambda^{2}}{1-q^{j} \Lambda^{2}}$ is the $q$, t-generalization of the HarishChandra c-function; $\langle\mu\rangle$ is from (2.1).

This identity follows from the $q, t-$ part of paper [Ch5] and a straightforward calculation of the expansion coefficients. The terms in the right-hand satisfy the Macdonald eigenvalue problem, so they can be extended meromorphically to all $X, \Lambda$.

Formula (2.29) is actually the result of the $R \mathbb{E}$ limiting procedure applied to (2.31); it is an instructional calculation. We replace $X$ by $X^{-1}$ (using that $F$ is symmetric) and then use that

$$
R E\left(\frac{\widetilde{\gamma}^{\prime}(X) \widetilde{\gamma}^{\prime}(\Lambda)}{\widetilde{\gamma}^{\prime}\left(t^{1 / 2}\right) \widetilde{\gamma}^{\prime}\left(X^{-1} \Lambda t^{-1 / 2}\right)}\right)=\frac{\widetilde{\gamma}^{\prime}(X) \widetilde{\gamma}^{\prime}(\Lambda)}{\widetilde{\gamma}^{\prime}\left(X^{-1} \Lambda\right)}
$$

It suffices to take here $q^{-x^{2}}$ instead of $\widetilde{\gamma}^{\prime}(X)$ (and for the other arguments). Then this formula becomes quite obvious. The limits of the other terms in (2.31) can be obtained by the straight substitution $t \mapsto 0$.

The left-hand side here is an analytic function for all $X, \Lambda$ when $|q|<1$, so its convergence is significantly better than for the classical basic hypergeometric series on the right-hand side. The parameter $t$ is assumed sufficiently general to avoid the zeros of $\left\langle P_{n}, P_{n}\right\rangle$. The inequality $|X|<|t|^{1 / 2}|q|^{-1 / 2}$ from the theorem ensures the convergence of the right-hand side for $|q|<1$.

Note that when $|q|>1$, the summations in the right-hand side still converge if we impose the condition $|X|<|q|^{1 / 2}|t|^{-1 / 2}$. Thus the right hand side can be used to connect the domains $|q|<1$ and $|q|>1$ (under the above inequality). Here the function $\sigma\left(\Lambda^{ \pm 1}\right)$ must be redefined 
using the $q$-exponential functions. This continuation actually goes through the unit circle.

Concerning the left-hand side, it can be continued analytically to

$$
|q|=1 \text { provided }\left|X^{ \pm 1} \Lambda^{ \pm 1}\right|<|t|^{1 / 2} R(q) .
$$

Compare with (2.30). The continuation is for sequences $\left\{q_{n}\right\}$ approaching $q$ such that $\left|1-q_{n}^{m}\right|^{1 / m}>\epsilon$ for an arbitrarily small $\epsilon>0$, almost all $m>0$, and sufficiently large $n$.

Recall that $F$ in the left hand-side remains a solution of the same difference equation $\mathcal{L}(F)=\left(\Lambda+\Lambda^{-1}\right) F$ upon the formal star-conjugation

$$
q \mapsto q^{-1}, t \mapsto t^{-1}, X \mapsto X^{-1}, \Lambda \mapsto \Lambda^{-1}
$$

This readily provides a formula defined for $|q|>1$. It is straightforward to calculate its expansion decomposition.

The connection between the regions $|q|<1$ and $|q|>1$ in the $q, t-$ case is significantly different from that in the Whittaker case. In the Whittaker case, the conjugation changes the corresponding difference equation (it does not in the $q, t$-case); however, the defining series of each of these two functions (the left-hand size of (2.29)) can be extended from the region $|q|<1$ to the region $|q|>1$ simply by recalculating the corresponding series in terms of $q^{-1}$.

Comment. Formula (2.31) (without explicit expressions for coefficients) was announced by Jasper Stokman for $G L(n)$ in his lecture (June, 2009). A complete theory of the $q, t$-version of the HarishChandra expansion and decomposition theory for any reduced root systems (the twisted case) is in his recent [Sto2]. Similarly to the classical differential theory, the difference decomposition formula results from the following two ingredients:

(a) the calculation of the corresponding asymptotic limit, which is the $c$-function ([Ch5] in the $q, t$-case),

(b) the coefficient-wise existence and uniqueness of the asymptotic series and its meromorphic continuation.

The formal uniqueness is essentially sufficient to establish the existence of this series in a neighborhood of infinity. The exponential growth of the coefficients results from the recurrence relations for them (the uniqueness). Then the corresponding differential or difference equations can be used for the meromorphic continuation.

Stokman's approach is based on the Cherednik-Matsuo map to/from the QAKZ-equation, where the calculation of the asymptotic coefficients is very similar to that in the differential AKZ-case (paper 
[Ch6] and previous first author's papers on the $r$-matrix KnizhnikZamolodchikov equations).

An alternative approach to the coefficient-wise existence and uniqueness of the asymptotic expansion is in deducing this fact from the differential theory, namely, from that due to Heckman and Opdam; see [HO], which is the limiting case $q \rightarrow 1$ of the $q, t$-theory. The standard deformation argument is used here. This line was suggested by Opdam and the first author.

Paper [MS] (the $G L(n)$-case) and the papers [Me, Sto2] (the twisted case, arbitrary reduced root systems) are devoted to the existence of the asymptotic series, their convergence and symmetries. We remark that paper [Ch5] was written in the twisted case too.

The advantage of the approach due to van Meer and Stokman (vs. that based on the deformation to the differential theory) is that the convergence of the asymptotic series in a neighborhood of infinity readily results in its global meromorphic continuation. The QAKZ is a difference system of equations, which readily provides the desired meromorphic continuation (in contrast to the differential theory).

The Macdonald eigenvalue problem (used directly) is expected to provide the same, but the corresponding tools are not properly developed at the moment.

A disadvantage of this approach is that the Cherednik-Matsuo map is the symmetrization of the vector-valued solutions of QAKZ (of dimension $|W|$ ), which makes it practically impossible to use for explicit calculation of the asymptotic coefficients (even in the simplest cases). Finding the fundamental solution of AQKZ is generally significantly more difficult problem than direct finding the symmetric solution of the Macdonald eigenvalue problem, algebraically and analytically (in spite of the fact that Cherednik-Matsuo maps are isomorphisms).

The Whittaker reduction of the $q, t$-version of the Harish-Chandra asymptotic decomposition formula and its connection with the GiventalLee theory was announced by the first author. It also establishes the connection with [GW] and [Wa] and with the classical $p$-adic Whittaker theory.

The first author is grateful to Jasper Stokman for the information about his ongoing research and Eric Opdam for useful discussions.

2.2.3. Nonsymmetric q-Hermite polynomials. For an $E$-polynomial $E_{n}$, let us define its two limits:

$$
\bar{E}_{n}=\lim _{t \rightarrow 0} E_{n} \text { and } \bar{E}_{n}^{\dagger}=\lim _{t \rightarrow \infty} E_{n} .
$$


Both limits exist (for instance, use the explicit formulas or the intertwining operators) and are closely connected to each other.

Proposition 2.4. For $n \geq 0$,

$$
\bar{E}_{-n}^{\dagger}=\left.\left(q^{\frac{n}{2}} \bar{E}_{-n}\left(X q^{\frac{1}{2}}\right)\right)\right|_{q \rightarrow q^{-1}}, \bar{E}_{n}^{\dagger}=\left.\left(q^{-\frac{n}{2}} \bar{E}_{n}\left(X q^{\frac{1}{2}}\right)\right)\right|_{q \rightarrow q^{-1}} .
$$

The polynomials $\bar{E}_{n}$ are called nonsymmetric (continuous) q-Hermite polynomials (see [Ch5] and references therein; they are considered there for arbitrary reduced root systems). Their symmetrizations are the classical $q$-Hermite polynomials.

Comment. Upon the substitution $X \mapsto X^{-1}$, the polynomials $\bar{E}_{n}$ are directly related to the Demazure characters of level one Kac-Moody integrable modules, thus are closely connected with the geometry of the affine Schubert varieties (through the Kumar-Mathieu formula).

This connection is from [San] in the $G L(n)$-case; for arbitrary root systems, it is from [Ion] (the twisted case).

More systematically, let us define

$$
\bar{T} \stackrel{\text { def }}{=} \lim _{t \rightarrow 0} t^{1 / 2} T=\frac{1}{1-X^{2}} \circ(s-1), \bar{T}(\bar{T}+1)=0 .
$$

Using intertwiners, $\overline{E_{0}}=1$,

$$
\begin{aligned}
& \bar{E}_{1+n}=q^{n / 2} \Pi \bar{E}_{-n}, \\
& \bar{E}_{-n}=(\bar{T}+1) \bar{E}_{n}
\end{aligned}
$$

for $n \geq 0$; the raising operator $\Pi=X \pi$ was defined in (1.15).

From the divisibility condition $\bar{T}+1=(s+1) \cdot\{\}$, we obtain that $\bar{E}_{-n}$ is symmetric (s-invariant) and $\bar{P}_{n}=\bar{E}_{-n}$ for $n \geq 0$.

Explicitly,

$$
\begin{gathered}
\bar{E}_{-n-1}=\left((\bar{T}+1) \Pi q^{n / 2}\right) \bar{E}_{-n}, \\
(\bar{T}+1) \Pi=\frac{X^{2} \Gamma^{-1}-X^{-2} \Gamma}{X-X^{-1}} .
\end{gathered}
$$

The bar-Pieri rules read as follows:

$$
\begin{aligned}
& X^{-1} \bar{E}_{-n}=\bar{E}_{-n-1}-\bar{E}_{n+1}(n \geq 0), \\
& X^{-1} \bar{E}_{n}=\left(1-q^{n-1}\right) \bar{E}_{n-1}+q^{n-1} \bar{E}_{1-n}(n \geq 1), \\
& X \bar{E}_{-n}=\left(1-q^{n}\right) \bar{E}_{1-n}+\bar{E}_{n+1}(n \geq 0), \\
& X \bar{E}_{n}=\bar{E}_{n+1}-q^{n} \bar{E}_{1-n}(n \geq 1) .
\end{aligned}
$$


Let $\bar{Y}=\pi \bar{T}=\lim _{t \rightarrow 0} t^{1 / 2} Y$. Recall that

$$
Y E_{n}=\left\{\begin{array}{cc}
t^{-1 / 2} q^{-n / 2} E_{n}, & n>0 \\
t^{1 / 2} q^{-n / 2} E_{n}, & n \leq 0
\end{array}\right.
$$

In the limit,

$$
\bar{Y} \bar{E}_{n}=\left\{\begin{array}{cc}
q^{-|n| / 2} \bar{E}_{n}, & n>0 \\
0, & n \leq 0
\end{array}\right.
$$

Since $\bar{Y}$ is not invertible, we need to introduce

$$
\bar{Y}^{\prime}=\lim _{t \rightarrow 0} t^{1 / 2} Y^{-1}=\lim _{t \rightarrow 0} t^{1 / 2} T^{-1} \pi=\bar{T}^{\prime} \pi
$$

for $\bar{T}^{\prime}=\bar{T}+1$. Then $\bar{Y} \bar{Y}^{\prime}=0=\bar{Y}^{\prime} \bar{Y}$ and

$$
\bar{Y}^{\prime} \bar{E}_{n}=\left\{\begin{array}{cc}
q^{-|n| / 2} \bar{E}_{n}, & n \leq 0, \\
0, & n>0 .
\end{array}\right.
$$

Finally,

$$
\overline{\mathcal{L}}=\lim _{t \rightarrow 0} t^{1 / 2} \mathcal{L}=\bar{Y}^{\prime}+\bar{Y}=\frac{1}{1-X^{2}} \Gamma+\frac{1}{1-X^{-2}} \Gamma^{-1}
$$

and $\overline{\mathcal{L}} \bar{P}_{n}=q^{-n / 2} \bar{P}_{n}, n \geq 0($ see $(1.27))$; recall that $\bar{P}_{n}=\bar{E}_{-n}$.

2.2.4. The series for the mu-function. The Hermite-type degeneration of the function $\mu$ from (1.10) is as follows:

$$
\begin{aligned}
& \bar{\mu}(X ; q)=\prod_{j=0}^{\infty}\left(1-q^{j} X^{2}\right)\left(1-q^{j+1} X^{-2}\right)= \\
& \prod_{j=1}^{\infty}\left(1-q^{j}\right)^{-1} \sum_{n=-\infty}^{\infty}(-1)^{n} q^{\frac{n^{2}-n}{2}} X^{2 n}, \\
& \langle\bar{\mu}\rangle=\prod_{j=1}^{\infty}\left(1-q^{j}\right)^{-1}, \omega(\bar{\mu})=\bar{\mu}\left(X q^{-1 / 2} ; q\right)=\left(-X^{2} q^{-1}\right) \bar{\mu} .
\end{aligned}
$$

Also, $\bar{\mu}\left(X^{-1}\right)=-X^{-2} \bar{\mu}(X)$. Compare with $\omega\left(\widetilde{\gamma}^{\prime}\right)=\left(q^{-1 / 4} X\right) \widetilde{\gamma}^{\prime}$, where

$$
\begin{aligned}
\widetilde{\gamma}^{\prime} & =\sum_{n=-\infty}^{\infty} q^{n^{2} / 4} X^{n} \\
& =\prod_{j=1}^{\infty}\left(1-q^{j / 2}\right)\left(1+q^{\frac{2 j-1}{4}} X\right)\left(1+q^{\frac{2 j-1}{4}} X^{-1}\right)
\end{aligned}
$$


The function $\widetilde{\gamma}^{\prime} \bar{\mu}$ serves the inner product with the Gaussian. One has from (2.4):

$$
\left\langle\widetilde{\gamma}^{\prime} \bar{\mu}_{\circ}\right\rangle=\prod_{j=1}^{\infty}\left(1-q^{j}\right),\left\langle\widetilde{\gamma}^{\prime} \bar{\mu}\right\rangle=1
$$

The complete expansion is as follows:

$$
\widetilde{\gamma}^{\prime} \bar{\mu}=\sum_{n=0}^{\infty} q^{n(n+2) / 12}\left(X^{n+2}-X^{-n}\right), \text { where } n \neq 2 \bmod 3 .
$$

More explicitly, the coefficient of $\left(X^{n+2}-X^{-n}\right)$ here equals

$$
q^{\frac{(3 m+2) m}{4}} \text { for } n=3 m, q^{\frac{(3 m+1)(m+1)}{4}} \text { for } n=3 m+1,0 \text { otherwise. }
$$

Ignoring $n \neq 2 \bmod 3$ and substituting $n \mapsto 2 n, q \mapsto q^{3 / 2}$, the summation in (2.43) becomes that from (2.40).

Adding the $\bar{E}$-polynomials, the limit of $(2.7)$ as $k \rightarrow \infty(t \rightarrow 0)$ is

$$
\begin{aligned}
& \left\langle\bar{E}_{n} \bar{E}_{m} \widetilde{\gamma}^{\prime} \bar{\mu}\right\rangle=\lim _{k \rightarrow \infty} q^{\frac{m^{2}+n^{2}+2 k|m|}{4}} E_{m}\left(q^{n_{\sharp}}\right)= \\
& q^{\frac{(m-n)^{2}}{4}} \text { for } \operatorname{sgn}(n)+\operatorname{sgn}(m)<2 \text { and } 0 \text { otherwise. }
\end{aligned}
$$

Indeed, the term $q^{\frac{k|m|}{2}} E_{m}\left(q^{n_{\sharp}}\right)$ is nonzero in the limit if and only if $E_{m}$ contains the monomial $X^{-\operatorname{sgn}(n)|m|}$. This holds unless $n>0$ and $m>0$. Then the coefficient of this monomial will be always 1 in the bar-limit; we use that $\bar{E}_{-n}=\bar{P}_{n}$ for $n>0$.

The particular cases $0 \leq n, m \leq 1$ are immediate from (2.43).

\section{NIL-DAHA}

3.1. Key definitions. A systematic theory of $q$-Hermite polynomials and global Whittaker functions begins with the following definition of the nil-DAHA which can be readily adjusted to arbitrary (reduced) root systems.

Definition 3.1. (i) The nil-DAHA $\overline{\mathcal{H}}$ is generated by $T, \pi, X^{ \pm 1}$ over the ring $\mathrm{C}\left[q^{ \pm 1 / 4}\right]$ with the defining relations: $T(T+1)=0$,

$$
\pi^{2}=1, \pi X \pi=q^{1 / 2} X^{-1}, T X-X^{-1} T=X^{-1} .
$$

Equivalently, $T X=X^{-1} T^{\prime}$ for $T^{\prime} \stackrel{\text { def }}{=} T+1$. Setting $Y \stackrel{\text { def }}{=} \pi T$ and $Y^{\prime} \stackrel{\text { def }}{=} T^{\prime} \pi$ one has: $T^{\prime} Y=Y^{\prime} T, T Y^{\prime}=0=Y T^{\prime}$. 
(ii) Similarly, one can define $\overline{\mathcal{H}}^{\varphi}=\mathrm{C}\left[q^{ \pm 1 / 4}\right]\left\langle T, \breve{\pi}, Y^{ \pm 1}\right\rangle$ subject to $T T^{\prime}=0$ for $T^{\prime}=T+1$ and

$$
\breve{\pi}^{2}=1, \breve{\pi} Y \breve{\pi}=q^{-1 / 2} Y^{-1}, T Y^{-1}=Y T^{\prime} .
$$

Setting $X \stackrel{\text { def }}{=} \breve{\pi} T^{\prime}, X^{\prime} \stackrel{\text { def }}{=} T \breve{\pi}$, one has: $T X=X^{\prime} T^{\prime}, T^{\prime} X^{\prime}=0=X T$.

(iii) The algebra $\overline{\mathcal{H}}^{\varphi}$ is the image of the algebra $\overline{\mathcal{H}}$ under the anti-isomorphism

$$
\varphi: T \mapsto T, \pi \mapsto \breve{\pi}, X \mapsto Y^{-1}
$$

Correspondingly, $\varphi: Y \mapsto X^{\prime}, Y^{\prime} \mapsto X$. There is also an isomorphism $\sigma: \overline{\mathcal{H}} \rightarrow \overline{\mathcal{H}}^{\varphi}$ sending

$$
\begin{aligned}
& \sigma: T \mapsto T, \quad X \mapsto Y^{-1}, \quad \pi \mapsto \breve{\pi}, \\
& \sigma: Y \mapsto \breve{\pi} T, \quad Y^{\prime} \mapsto T^{\prime} \breve{\pi} .
\end{aligned}
$$

(iv) The automorphism $\tau_{+}$fixing $T, X$ and sending $Y \mapsto q^{-1 / 4} X Y$ acts in $\overline{\mathcal{H}}$. Correspondingly, $\tau_{-} \stackrel{\text { def }}{=} \varphi \tau_{+} \varphi^{-1}$ acts in $\overline{\mathcal{H}}^{\varphi}$ preserving $T, Y$ and sending $X \mapsto q^{1 / 4} Y X$. One has the relations

$$
\sigma \tau_{+}=\tau_{-}^{-1} \sigma, \sigma \tau_{+}^{-1}=\tau_{-} \sigma
$$

matching the identity from (1.7) in the generic case.

Both algebras, $\overline{\mathcal{H}}$ and $\overline{\mathcal{H}}^{\varphi}$, satisfy the PBW Theorem because they are limits of $\mathcal{H}$ (to be discussed below in detail), so the $q, t$-algebra is their flat deformation. It includes the case when $q$ is a root of unity.

3.1.1. Polynomial representation. Any $H \in \overline{\mathcal{H}}$ can be represented as $H=\sum_{i} c_{i} X^{i} h_{i}$ for constant $c_{i}(i \in \mathrm{z})$ and $h_{i} \in \overline{\mathcal{H}}_{Y} \stackrel{\text { def }}{=}<T, \pi>$; this readily follows from the defining relations. Moreover, this representations is unique since $\overline{\mathcal{H}}$ is a limit of $\mathcal{H}$, where the PBW Theorem holds. The existence of the above representations automatically guarantees their uniqueness. Furthermore, any element $h \in \overline{\mathcal{H}}_{Y}$ can be uniquely expressed as a linear combination of

$$
Y^{m} \pi,\left(Y^{\prime}\right)^{m+1} \pi, Y^{m},\left(Y^{\prime}\right)^{m+1} \text { where } m \geq 0 .
$$

The bar-polynomial representation $\overline{\mathscr{X}}$ is $\operatorname{Ind} \frac{\overline{\mathcal{H}}_{\mathcal{H}_{Y}}}{\mathrm{C}_{+}^{0}}=\mathrm{C}\left[q^{ \pm 1 / 4}\right]\left[X^{ \pm 1}\right]$, where $\mathrm{C}_{ \pm}^{0,1}$ are one-dimensional representations of $\overline{\mathcal{H}}_{Y}$ defined as follows:

$$
\begin{aligned}
& T^{\prime}(1)=\epsilon, T(1)=\epsilon-1, \pi(1)= \pm 1 \\
& Y(1)= \pm(\epsilon-1), Y^{\prime}(1)= \pm \epsilon \text { for } \epsilon=0,1 .
\end{aligned}
$$


The bar-formulas discussed above give an explicit description of the polynomial (or bar-polynomial) representation of $\overline{\mathcal{H}}$ in $\overline{\mathscr{X}}$; recall that $T, \pi, X^{ \pm 1}, Y, Y^{\prime}$ are mapped to the operators $\bar{T}, \pi, X^{ \pm 1}, \bar{Y}, \bar{Y}^{\prime}$.

3.1.2. The order function. An important feature of the nil-case is that the nil-DAHA are filtered algebras with Weyl-type algebras as their associated graded algebras. It significantly simplifies the PBW Theorem and related issues. Let the order be $m$ for the following elements

$$
X^{l} Y^{m} \pi, X^{l}\left(Y^{\prime}\right)^{m} \pi(m>0), X^{l} Y^{m}, X^{l}\left(Y^{\prime}\right)^{m}(m>0),
$$

where $l \in \mathrm{Z}, m \in \mathrm{Z}_{+}$. The order $\operatorname{ord}(H)$ of $H \in \overline{\mathcal{H}}$ will be defined as the maximum of orders of the terms in the linear decomposition of $H$ with respect to (3.6).

Proposition 3.2. The order above satisfies

$$
\operatorname{ord}\left(H_{1} H_{2}\right) \leq \operatorname{ord}\left(H_{1}\right)+\operatorname{ord}\left(H_{2}\right) .
$$

The associated graded algebra gr $\overline{\mathcal{H}}$ is isomorphic to

$$
\overline{\mathcal{W}} \stackrel{\text { def }}{=}<X, X^{-1}, Y, Y^{\prime}, \pi>\text { over } \mathrm{C}\left[q^{ \pm 1 / 4}\right]
$$

subject to nil-versions of the Weyl-type relations

$$
\begin{array}{r}
Y Y^{\prime}=0=Y^{\prime} Y, X Y=q^{1 / 2} Y X \text { extended by } \\
\pi^{2}=1, \pi X \pi=q^{1 / 2} X^{-1}, \pi Y \pi=Y^{\prime} .
\end{array}
$$

They result in $T^{2}=0$ for $T=\pi Y=Y^{\prime} \pi$ and in $X Y^{\prime}=q^{-1 / 2} Y^{\prime} X$. The corresponding grading is given by $\operatorname{ord}(Y)=1=\operatorname{ord}\left(Y^{\prime}\right), \operatorname{ord}(X)=$ $0=\operatorname{ord}(\pi)$.

Proof. The relations $Y^{\prime} T=T^{\prime} Y, Y^{\prime}=T^{\prime} \pi$ become $Y T=T Y$ and $Y^{\prime}=T \pi$ in $\operatorname{gr} \overline{\mathcal{H}}$ since $T^{\prime}=T$ in the latter. Similarly, $T X=X^{-1} T^{\prime}$ becomes $X Y=q^{1 / 2} Y X$ upon the substitution $T=\pi Y=T^{\prime}$.

3.1.3. Invariant symmetric forms. Recall (2.40):

$$
\bar{\mu}(X ; q)=\prod_{j=0}^{\infty}\left(1-q^{j} X^{2}\right)\left(1-q^{j+1} X^{-2}\right) .
$$

The inner products

$$
\langle f, g\rangle_{\circ}=\left\langle f g \mu_{\circ}\right\rangle,\langle f, g\rangle^{\prime}=\left\langle f g \widetilde{\gamma}^{\prime} \mu\right\rangle .
$$

from (1.11) can obviously be used in the bar-polynomial representation $\overline{\mathscr{X}}$. The remaining form, which involves the conjugation, will be 
addressed later. The kernels of these bilinear forms are the bar-limits, $\bar{\mu}_{\circ}$ and $\widetilde{\gamma} \bar{\mu}$ from (2.40) and (2.43).

Note that $\langle 1,1\rangle_{\circ}=1=\langle 1,1\rangle^{\prime}$ and both forms are actually welldefined for any $q$ (including roots of unity). One can see this directly from (2.40) for $\langle f, g\rangle_{\circ}$. The second inner product was calculated in (2.44):

$$
\begin{aligned}
& \left\langle\bar{E}_{n}, \bar{E}_{m}\right\rangle^{\prime}= \\
& q^{\frac{(m-n)^{2}}{4}} \text { for } \operatorname{sgn}(n)+\operatorname{sgn}(m)<2 \text { and } 0 \text { otherwise. }
\end{aligned}
$$

The corresponding anti-involutions of the algebra $\overline{\mathcal{H}} \ni H$ are those from $(1.8,1.9)$ :

$$
\langle f, H(g)\rangle_{\circ}=\left\langle H^{\diamond}(f), g\right\rangle_{\circ},\langle f, H(g)\rangle^{\prime}=\left\langle H^{\psi}(f), g\right\rangle^{\prime},
$$

where $f, g \in \overline{\mathscr{X}}, H^{\psi}=\psi(H)$.

3.2. Using the conjugation. The nil-DAHA is a limit of $\mathcal{H} \mathcal{H}$ as $t \rightarrow 0$. The anti-involution $*$ plays an important role in the $q, t$-theory. Since $t^{*}=t^{-1}$, its nil-counterpart requires considering the limit as $t \rightarrow \infty$ as well.

3.2.1. The limit at infinity. We use $\overline{\mathcal{H}}^{\dagger}$ to denote the resulting algebra. Explicitly, we have $\overline{\mathcal{H}}^{\dagger}=<T^{\dagger}, \pi, X^{ \pm 1}>$ subject to the defining relations:

$$
\begin{aligned}
& \left(T^{\dagger}-1\right) T^{\dagger}=0, \pi^{2}=1, \pi X \pi=q^{1 / 2} X^{-1} \\
& T^{\dagger} X=X^{-1}\left(T^{\dagger}-1\right)=X^{-1}\left(T^{\dagger}\right)^{\prime} \text { for }\left(T^{\dagger}\right)^{\prime} \stackrel{\text { def }}{=} T^{\dagger}-1 .
\end{aligned}
$$

We set $T^{\dagger}=\lim _{t \rightarrow \infty} t^{-1 / 2} T$ in the limit from $\mathcal{H}$. The polynomial representation $\mathscr{X}$ survives in this limit; we denote the resulting $\overline{\mathcal{H}}^{\dagger}-$ module by $\overline{\mathscr{X}}^{\dagger}$. The operators $\pi, X^{ \pm 1}$ remain the same, but $T^{\dagger}$ acts by

$$
\bar{T}^{\dagger}=s+\frac{1}{X^{2}-1}(s-1) .
$$

The anti-involution $*$ naturally becomes the anti-isomorphism sending

$$
\begin{aligned}
& \overline{\mathcal{H}} \ni H \mapsto H^{*} \in \overline{\mathcal{H}}^{\dagger}, \\
& T \mapsto\left(T^{\dagger}\right)^{\prime}=T^{\dagger}-1, \pi \mapsto \pi, X^{ \pm 1} \mapsto X^{\mp 1}, q \mapsto q^{-1} .
\end{aligned}
$$

Its inverse will be denoted by the same symbol; we note that $\left(T^{\dagger}\right)^{*}=$ $T^{\prime}=T+1$ under this map. 
3.2.2. Connection maps. These two algebras are connected by the following $\mathrm{C}\left[q^{ \pm 1 / 4}\right]$-linear isomorphisms:

$$
\begin{aligned}
& \nu_{ \pm}: \overline{\mathcal{H}} \rightarrow \overline{\mathcal{H}}^{\dagger}: T \mapsto-T^{\dagger}, \pi \mapsto \pm \pi, X \mapsto X, \\
& \beta: \overline{\mathcal{H}} \rightarrow \overline{\mathcal{H}}^{\dagger}: T \mapsto X^{-2}\left(1-T^{\dagger}\right), \pi \mapsto \pi, X \mapsto X .
\end{aligned}
$$

The map $\beta$ is a adjusted to the polynomial representations in the following sense: for any $f \in \mathrm{C}\left[q^{ \pm 1 / 4}\right]\left[X^{ \pm 1}\right]$ and $H \in \overline{\mathcal{H}}$ we have

$$
\overline{\mathscr{X}} \ni \bar{H}(f)=\overline{\beta(H)}(f) \in \overline{\mathscr{X}}^{\dagger}, \text { for instance, }
$$

$$
\bar{T}=\frac{1}{1-X^{2}}(s-1)=X^{-2}\left(1-\bar{T}^{\dagger}\right)=X^{-2}\left(1-s+\frac{1}{1-X^{2}}(s-1)\right) .
$$

See (2.34); we continue to use bar for the operators acting in the standard polynomial representation.

We note that $\nu_{+}^{-1} \beta$ and $\beta \nu_{+}^{-1}$ are the automorphisms of $\overline{\mathcal{H}}$ and $\overline{\mathcal{H}}^{\dagger}$ given by conjugation by $X^{-1}$ followed by $\tau_{+}^{2}$. For instance, in $\overline{\mathcal{H}}$ we have the identity

$$
X^{-1} T X=X^{-2} T+X^{-2},
$$

and by applying $\nu_{+}$to the right-hand side we obtain the image of $T$ under $\beta$ ( $T, X$ are fixed under $\tau_{+}$, which we need here for $\pi$ only).

Finally, we define an isomorphism

$$
\begin{aligned}
& \eta: \overline{\mathcal{H}} \rightarrow \overline{\mathcal{H}}^{\dagger}: \\
& T \mapsto\left(T^{\dagger}\right)^{\prime}=T^{\dagger}-1, \pi \mapsto \pi, X \mapsto X^{-1}, q^{1 / 4} \mapsto q^{-1 / 4},
\end{aligned}
$$

and its inverse, sometimes denoted by the same symbol $\eta$. This definition is a direct nil-variant of that from (2.19).

Proposition 3.3. There exists a unique C-linear automorphism denoted by $*$ from $\overline{\mathscr{X}}$ to $\overline{\mathscr{X}}^{\dagger}$ and the one in the opposite direction sending $1 \mapsto 1, q \mapsto q^{-1}$ and compatible with $\eta$ :

$$
\begin{aligned}
& \overline{\mathscr{X}} \ni H\left(f^{*}\right)=(\eta(H)(f))^{*} \in \overline{\mathscr{X}}^{\dagger} \quad \text { for } \quad H \in \overline{\mathcal{H}}, \\
& \overline{\mathscr{X}}^{\dagger} \ni H\left(f^{*}\right)=(\eta(H)(f))^{*} \in \overline{\mathscr{X}} \quad \text { for } \quad H \in \overline{\mathcal{H}}^{\dagger} .
\end{aligned}
$$

Upon the standard identification of the polynomial representations with $\mathrm{C}\left[q^{ \pm 1 / 4}\right]\left[X^{ \pm 1}\right]$, it becomes the conjugation, namely, $\left(X^{n}\right)^{*}=X^{-n}$ for all $n$ and $q^{1 / 4} \mapsto q^{-1 / 4}$. Moreover,

$$
\left(\bar{E}_{n}\right)^{*}=X^{-1} \bar{E}_{1-n}^{\dagger} \text { and }\left(\bar{E}_{n}^{\dagger}\right)^{*}=X^{-1} \bar{E}_{1-n} \text { for } n \in \mathrm{Z} .
$$


3.2.3. Pairing with conjugation. The remaining inner product from formula (1.11) in the o-normalization is:

$$
((f, g))_{\circ}=\left\langle f g^{*} \mu_{\circ}\right\rangle .
$$

In the limit, it becomes a pairing between $f \in \overline{\mathscr{X}}$ and $g \in \overline{\mathscr{X}}^{\dagger}$ (or in the opposite order):

$$
((f, g))_{\circ}=\left\langle f g^{*} \bar{\mu}_{\circ}\right\rangle, \quad((1,1))_{\circ}=1, \quad((g, f))_{\circ}=\left\langle g f^{*} \bar{\mu}_{\circ}^{\dagger}\right\rangle=((f, g))_{\circ}^{*} .
$$

Here $g^{*} \in \overline{\mathscr{X}}$, so $\mu_{\circ}$ in the first formula becomes $\bar{\mu}_{\circ}$, which is the limit of $\mu_{\circ}$ as $t \rightarrow 0$. In the opposite order, $\bar{\mu}_{\circ}^{\dagger}=\lim _{t \rightarrow \infty} \mu_{\circ}$ must be used; see (1.28).

Check that

$$
\lim _{t \rightarrow 0}\left(g^{*}\right)=\left(\lim _{t \rightarrow \infty} g\right)^{*}
$$

whenever the limits exist. Recall that $\overline{\mathscr{X}}$ and $\overline{\mathscr{X}}^{\dagger}$ are both equal to $\mathrm{C}\left[q^{ \pm 1 / 4}\right]\left[X^{ \pm 1}\right]$, but the former is defined as a module over $\overline{\mathcal{H}}$, the latter over $\overline{\mathcal{H}}^{\dagger}$.

Taking the limit of the first formula in (1.12), we obtain

$$
((H(f), g))_{\circ}=\left(\left(f, H^{*}(g)\right)\right)_{\circ},
$$

where $H \in \overline{\mathcal{H}}$ and hence $H^{*} \in \overline{\mathcal{H}}^{\dagger}$.

For instance, let us consider $T$. Then in $\mathcal{H}$ :

$$
\left\langle T(f) g^{*} \mu_{\circ}\right\rangle=((T(f), g))_{\circ}=\left(\left(f, T^{*}(g)\right)\right)_{\circ}=\left\langle f\left(T^{-1}(g)\right)^{*} \mu_{\circ}\right\rangle .
$$

This relation must be multiplied by $t^{1 / 2}$ followed by $t \rightarrow 0$. On the left-hand side $t^{1 / 2} T \rightarrow \bar{T}$, while on the far right-hand side we need to consider the limit of $t^{1 / 2}\left(T^{-1}(g)\right)^{*}=\left(t^{-1 / 2} T^{-1}(g)\right)^{*}$. Moving inside $*$, $t^{-1 / 2} T^{-1}$ tends to $T^{\dagger}-1$ as $t \rightarrow \infty$. Thus

$$
((\bar{T}(f), g))_{\circ}=\left(\left(f,\left(T^{\dagger}-1\right)(g)\right)\right)_{\circ} .
$$

We note that $(1.24)$ readily results in

$$
\begin{aligned}
& \left(\left(\bar{E}_{m}, \bar{E}_{n}^{\dagger}\right)\right)_{\circ}=\delta_{m n} \prod_{0<j<|\widetilde{n}|}\left(1-q^{j}\right), \\
& \left(\left(\bar{E}_{m}^{\dagger}, \bar{E}_{n}\right)\right)_{\circ}=\delta_{m n} \prod_{0<j<|\widetilde{n}|}\left(1-q^{-j}\right) .
\end{aligned}
$$


3.3. Tilde-subalgebras. A surprising fact is that the construction of non-symmetric Whittaker functions naturally leads to a module over $\overline{\mathcal{H}}^{\varphi}$, which differs significantly from the bar-polynomial representation. We will call it the hat-polynomial or spinor representation. It naturally appears within the technique of spinors to be discussed later.

The spinor representation is not a standard induced $\overline{\mathcal{H} H}$-module, but can be interpreted as a sub-induced module. The problem is that the subalgebra $\overline{\mathcal{H}}_{X}=<T, X^{ \pm 1}>$ has no one-dimensional representations and we need to diminish $\overline{\mathcal{H}}$ by switching from $X^{ \pm 1}$ to other (noninvertible) generators. It can be addressed as follows.

3.3.1. Alternative presentations. Let us begin with the following remark. Motivated the theory of the DAHA in the $q, t$-case, one can try to eliminate $\pi$ from the definition of nil-DAHA. It is doable, but less useful than in the general case.

The claim is that $\overline{\mathcal{H}}=<T, X^{ \pm 1}, Y>$. The remaining elements $\pi, Y^{\prime}$ are expressed as follows:

$$
\pi=q^{1 / 2} X^{-1} Y X-Y, Y^{\prime}=T^{\prime} \pi=T^{\prime}\left(q^{1 / 2} X^{-1} Y X-Y\right) .
$$

In terms of $T, X, Y$, the defining relations will be

$$
\begin{aligned}
& T(T+1)=0, \pi^{2}=\left(q^{1 / 2} X^{-1} Y X-Y\right)^{2}=1, \\
& Y\left(X+X^{-1}\right)=\left(q^{1 / 2} X^{-1}+q^{-1 / 2} X\right) Y, \\
& X\left(Y+Y^{\prime}\right)=\left(q^{1 / 2} Y+q^{-1 / 2} Y^{\prime}\right) X .
\end{aligned}
$$

However, the algebra $\overline{\mathcal{H}}$ does not have the PBW-property in terms of $T, X, Y$ (which is the key fact in the $q, t$-case). Also, as noted above, the affine Hecke subalgebra $\overline{\mathcal{H}}_{X}=<T, X^{ \pm 1}>$ has no one-dimensional representation due to the invertibility of $X$.

One may also consider $\overline{\mathcal{H}}$ as the algebraic span $\overline{\mathcal{H}}=<T, \pi, \widetilde{\pi}>$ over $\mathrm{C}\left[q^{ \pm 1 / 4}\right]$. The defining relations in this presentation are as follows:

$$
\begin{aligned}
& T(T+1)=0, \pi^{2}=1=\tilde{\pi}^{2}, \\
& \pi X \pi=q^{1 / 2} X^{-1} \text { for } X \stackrel{\text { def }}{=} q^{1 / 4} \tilde{\pi} \pi, \\
& \tilde{\pi} Y \widetilde{\pi}=q^{-1 / 2} Y^{\prime} \text { for } Y \stackrel{\text { def }}{=} \pi T .
\end{aligned}
$$

The function $\operatorname{ord}(H)$ becomes $\max _{i}\left\{\operatorname{ord}\left(N_{i}\right)\right\}$ for any reduced expression $H=\sum_{i} c_{i} N_{i}$, where $N_{i}$ are products (monomials) of $T, \pi, \tilde{\pi}$ and where $\operatorname{ord}\left(N_{i}\right)$ is the number of $T$ in the word for $N_{i}$. By reduced, we mean that the sum $H=\sum_{i} c_{i} N_{i}$ must have the least possible $\max _{i}\left\{\operatorname{ord}\left(N_{i}\right): c_{i} \neq 0\right\}$ among all such expressions for $H$ in terms of the products of $T, \pi, \widetilde{\pi}$. 
The embedding of $\overline{\mathcal{H}}$ into the abstract algebra defined in (3.31) is straightforward; the formulas for the images of $X, Y$ are provided there. It is obviously an isomorphism. The element $\widetilde{\pi}$ is actually from (1.8):

$$
\widetilde{\pi} \stackrel{\text { def }}{=} \tau_{+}(\pi)=q^{-1 / 4} X \pi=q^{1 / 4} \pi X^{-1} .
$$

3.3.2. Transitional subalgebras. The approach we need in order to address the spinor representation is actually opposite to the previous remarks; we need to eliminate $\pi, \tilde{\pi}, X$ from the list of generators. Let us introduce two proper subalgebras of $\overline{\mathcal{H}}$ :

$$
\widetilde{\mathcal{H}}_{\widetilde{\pi}, Y} \stackrel{\text { def }}{=}<T, \widetilde{\pi}, Y, Y^{\prime}>, \widetilde{\mathcal{H}}_{\pi, \widetilde{X}} \stackrel{\text { def }}{=}<T, \pi, \widetilde{X}, \widetilde{X}^{\prime}>,
$$

where

$$
\begin{aligned}
& \tilde{X} \stackrel{\text { def }}{=} \widetilde{\pi} T^{\prime}=q^{1 / 4} Y X, \\
& \tilde{X}^{\prime} \stackrel{\text { def }}{=} T \widetilde{\pi}=q^{-1 / 4} X^{-1} Y^{\prime} .
\end{aligned}
$$

These elements are direct nil-counterparts of $\widetilde{X}^{ \pm 1}$ used in (1.9). The anti-involution $\psi$ defined there acts in $\overline{\mathcal{H}}$ as does $\tau_{+}$; note that $\psi \tau_{+} \psi=$ $\tau_{+}^{-1}$.

One has:

$$
\begin{aligned}
& \psi\left(\widetilde{\mathcal{H}}_{\tilde{\pi}, Y}\right)=\widetilde{\mathcal{H}}_{\pi, \widetilde{X}} \text {, where } \psi: \\
& Y \mapsto \widetilde{X}^{\prime}, Y^{\prime} \mapsto \widetilde{X}, \widetilde{\pi} \mapsto \pi, T \mapsto T .
\end{aligned}
$$

The following relations hold:

$$
\tilde{X} T=0=T^{\prime} \tilde{X}^{\prime}, T \widetilde{X}=\tilde{X}^{\prime} T^{\prime} .
$$

3.3.3. Defining relations. We claim that $\widetilde{\mathcal{H}}_{\widetilde{\pi}, Y}$, considered as an abstract algebra, has the following defining relations:

$$
\begin{aligned}
& T(T+1)=0, \tilde{\pi}^{2}=1, \tilde{\pi} Y \tilde{\pi}=q^{-1 / 2} Y^{\prime} \\
& Y Y^{\prime}=0=Y^{\prime} Y, Y T^{\prime}=0=T Y^{\prime}, T^{\prime} Y=Y^{\prime} T, T^{\prime} \stackrel{\text { def }}{=} T+1
\end{aligned}
$$

Applying $\psi$, the defining relations of $\widetilde{\mathcal{H}}_{\pi, \widetilde{X}}$ are then

$$
\begin{aligned}
& T(T+1)=0, \pi^{2}=1, \pi \widetilde{X} \pi=q^{1 / 2} \widetilde{X}^{\prime}, \\
& \widetilde{X} \widetilde{X}^{\prime}=0=\widetilde{X}^{\prime} \widetilde{X}, \widetilde{X} T=0=T^{\prime} \widetilde{X}^{\prime}, T \widetilde{X}=\widetilde{X}^{\prime} T^{\prime} .
\end{aligned}
$$


It is obvious that all these relations hold in $\overline{\mathcal{H}}$. What is less obvious is that the corresponding homomorphisms

$$
\widetilde{\mathcal{H}}_{\widetilde{\pi}, Y} \rightarrow \overline{\mathcal{H}} \leftarrow \widetilde{\mathcal{H}}_{\pi, \widetilde{X}}
$$

are injective if these algebras are defined by the presentations above, not as subalgebras. This follows from Theorem 3.4, $(i, i i)$ below.

Comment. The standard way to verify the injectivity in (3.40) and to check similar facts is as follows.

(a) First of all, one must check that the abstract algebras from $(3.38,3.39)$ satisfy the claims of Theorem 3.4 below, except for the uniqueness part there, i.e., without verification of the linear independence of the terms listed there. Then one defines the tilde-polynomial representations of these algebras as proper induced modules (see below).

(b) Assuming that the uniqueness holds, the formulas for the generators acting in the tilde-representations can be calculated. Then one verifies directly that these formulas really give representations of the corresponding algebras, so these representations can be defined explicitly without any reference to the induction construction.

(c) Next, it is not difficult to check that the terms listed in Theorem 3.4 really are linearly independent as operators acting in the corresponding tilde-polynomial representations for generic $q$. This readily gives the uniqueness claims from this theorem for all $q$ and, finally, the injectivity of the maps in (3.40).

The nil-case. Using the order in nil-Hecke algebras simplifies the considerations versus the $q, t$-case; it can be used instead of the order of operators needed in (c). This order readily results in the existence of the PBW-type decompositions of the elements in $\overline{\mathcal{H}}$. The fact that the nil-algebras are limits of $\mathcal{H} \mathcal{H}$ (where the PBW Theorem holds) gives the uniqueness; the latter results in the injectivity from (3.40).

3.4. PBW and filtrations. The next step will be the PBW Theorems for the tilde-algebras.

Theorem 3.4. (tilde-PBW) (i) An arbitrary $H \in \widetilde{\mathcal{H}}_{\widetilde{\pi}, Y}$ can be uniquely represented as a linear combination of the terms

$\left(\tilde{X}^{\prime}\right)^{l} T Y^{m}, \tilde{X}^{l} \widetilde{\pi}\left(Y^{\prime}\right)^{m}, \tilde{\pi} Y^{m}(m>0), \tilde{X}^{l} Y^{m},\left(\tilde{X}^{\prime}\right)^{l}\left(Y^{\prime}\right)^{m}(l+m>0)$,

where $l \geq 0, m \geq 0$. 
(ii) An arbitrary $H \in \widetilde{\mathcal{H}}_{\pi, \widetilde{X}}$ can be uniquely represented as a linear combination of the terms

$\left(Y^{\prime}\right)^{l} T^{\prime} \tilde{X}^{m}, Y^{l} \pi\left(\tilde{X}^{\prime}\right)^{m}, \pi \tilde{X}^{m}(m>0), Y^{l} \tilde{X}^{m},\left(Y^{\prime}\right)^{l}\left(\tilde{X}^{\prime}\right)^{m}(l+m>0)$,

where $l \geq 0, m \geq 0$.

Proof. Let us check $(i)$. We can move $Y$ and $Y^{\prime}$ to the right (through $T$ and $\tilde{\pi}$ modulo lower terms). Therefore, any $H \in \widetilde{\mathcal{H}}_{\widetilde{\pi}, Y}$ can be represented as a linear combination of the terms $\widetilde{M} M, \widetilde{M} \widetilde{\pi} M$ and $\widetilde{M T} M$ for the monomials in the form $\widetilde{M}=\widetilde{X}^{l}$ or $\widetilde{M}=\left(\widetilde{X}^{\prime}\right)^{l}$ and $M=Y^{m}$ or $M=\left(Y^{\prime}\right)^{m}$, where $l, m \geq 0$. Further reductions are based on vanishing properties of the products of the generators and induction with respect to the degree defined by $l+m$ (i.e., we can disregard $T, \pi$ when they appear in the expressions).

Since $\widetilde{X} T=0$ and $T Y^{\prime}=0$, the terms $\left(\widetilde{X}^{\prime}\right)^{l} T Y^{m}$ are sufficient among those with $T$ in the middle. Next,

$$
\tilde{X} Y^{\prime}=\tilde{\pi} T^{\prime} T^{\prime} \pi=\tilde{\pi} T^{\prime} \pi=\tilde{\pi} Y^{\prime}, \tilde{X}^{\prime} \tilde{\pi}=T \tilde{\pi}^{2}=T
$$

which makes the terms $\widetilde{X}^{l}\left(Y^{\prime}\right)^{m}(m>0),\left(\tilde{X}^{\prime}\right)^{l} \widetilde{\pi} Y^{m}$ and $\left(\widetilde{X}^{\prime}\right)^{l} \widetilde{\pi}\left(Y^{\prime}\right)^{m}$ unnecessary modulo the terms of lower degree. Now

$$
\begin{aligned}
& \widetilde{X}^{\prime} Y=T \widetilde{\pi} Y=q^{-1 / 2} T Y^{\prime} \widetilde{\pi}=0, \\
& Y^{\prime} \widetilde{X}=T^{\prime} \pi \widetilde{X}=q^{1 / 2} T^{\prime} \widetilde{X}^{\prime} \pi=0 .
\end{aligned}
$$

The first of these identities completes the existence part. See (4.20) for more discussion concerning the degree. The uniqueness formally follows from the existence and the fact that $\overline{\mathcal{H}}$ is a limit of $\mathcal{H}$, which satisfies the PBW Theorem.

Note that the existence claim can readily obtained from the existence part of Proposition 3.5 below and can be also deduced from the explicit formulas for the generators acting in the tilde-polynomial representations (see the comment above).

Claim (ii) is very much similar. Let us list the key identities necessary in this case:

$$
\begin{aligned}
& Y^{\prime} \tilde{X}=Y \tilde{X}^{\prime}=Y T^{\prime} \tilde{X}=Y^{\prime} \pi \tilde{X}^{\prime}=0, \\
& Y \tilde{X}^{\prime}=-\pi \tilde{X}^{\prime}, Y \pi \widetilde{X}=-\tilde{X}, Y^{\prime} \pi=T^{\prime} .
\end{aligned}
$$


3.4.1. Using the order. A more conceptual way for normal ordering of the operators described in the theorem is based on the following proposition.

Proposition 3.5. Let ord $(N)$ be the number of $Y, Y^{\prime}, T, T^{\prime}$ in the elements (words) $N$ listed in (i) or in (ii). We set

$$
\mathcal{F}_{m} \stackrel{\text { def }}{=}\left\{\sum_{i} c_{i} N_{i}: \operatorname{ord}\left(N_{i}\right) \leq m\right\}
$$

Then $H_{1} H_{2} \in \mathcal{F}_{m_{1}+m_{2}}$ for $H_{1} \in \mathcal{F}_{m_{1}}$ and $H_{2} \in \mathcal{F}_{m_{2}}$. The graded algebras gr $\widetilde{\mathcal{H}}=\oplus_{m=0}^{\infty} \mathcal{F}_{m+1} / \mathcal{F}_{m}$ of $\widetilde{\mathcal{H}}_{\widetilde{\pi}, Y}$ and $\widetilde{\mathcal{H}}_{\pi, \tilde{X}}$ are correspondingly

$$
\begin{aligned}
& \widetilde{\mathcal{W}}_{\tilde{\pi}, Y} \stackrel{\text { def }}{=}<Y, Y^{\prime}, \widetilde{X}, \widetilde{X}^{\prime}, \tilde{\pi}>, \\
& \widetilde{\mathcal{W}}_{\pi, \widetilde{X}} \stackrel{\text { def }}{=}<Y, Y^{\prime}, \widetilde{X}, \widetilde{X}^{\prime}, \pi>
\end{aligned}
$$

subject to the corresponding Weyl-type relations

$$
\begin{gathered}
\pi^{2}=1, \tilde{\pi}^{2}=1, \quad Y Y^{\prime}=0=Y^{\prime} Y, \tilde{X} \tilde{X}^{\prime}=0=\tilde{X}^{\prime} \widetilde{X}, \\
Y \widetilde{X}^{\prime}=0=\tilde{X} Y^{\prime}, \quad \tilde{\pi} \widetilde{X}=\widetilde{X}^{\prime} \widetilde{\pi}, \pi Y=Y^{\prime} \pi, \\
\pi \widetilde{X}=q^{1 / 2} \widetilde{X}^{\prime} \pi, \quad \tilde{\pi} Y=q^{-1 / 2} Y^{\prime} \tilde{\pi}, \quad \widetilde{X} Y=q^{1 / 2} Y \widetilde{X} .
\end{gathered}
$$

In particular, it follows that $N \notin \mathcal{F}_{m-1}$ if $\operatorname{ord}(N)=m(m>0)$ for any element $N$ from the theorem, i.e., these elements are exactly of order $\operatorname{ord}(N)$ with respect to the filtration $\left\{\mathcal{F}_{i}\right\}$.

Proof. The procedure for taking $g r$ is as follows. We rescale the elements $N$ from the theorem: $N=h^{-\operatorname{ord}(N)} N(h)$, for instance, $T=$ $h^{-1} T(h)$. Then we send $h \rightarrow 0$; for instance, $\left(h^{-1} T(h)\right)^{2}=h^{-1} T(h)+1$ results in $T^{2}=0$ for $T=T(0)$ in $\operatorname{gr} \widetilde{\mathcal{H}}$. Thus $T$ and $T^{\prime}$ coincide under $g r, \pi Y=T=T^{\prime}=Y^{\prime} \pi$ and $\tilde{\pi} \widetilde{X}=T^{\prime}=T=\tilde{X}^{\prime} \tilde{\pi}$. Similarly, $\widetilde{X} Y^{\prime}=\widetilde{\pi} T^{\prime} T^{\prime} \pi=0$ in gr $\widetilde{\mathcal{H}}$ and

$$
\widetilde{X} Y=\widetilde{X} \pi T=q^{1 / 2} \pi \widetilde{X}^{\prime} T^{\prime}=q^{1 / 2} \pi T \widetilde{X}=q^{1 / 2} Y \widetilde{X} .
$$

Conjugating by $\tilde{\pi}$ and $\pi$, we obtain the remaining relations in gr $\widetilde{\mathcal{H}}$ :

$$
\tilde{X}^{\prime} Y=0=Y \tilde{X}^{\prime}, \tilde{X}^{\prime} Y^{\prime}=q^{1 / 2} Y^{\prime} \tilde{X}^{\prime}
$$

We see that the operators $\tilde{X}, Y$ and $\widetilde{\pi}$ or $\pi$ can be normally ordered in $g r$. The uniqueness, the fact that the relations from the proposition are really defining, follows from the fact that $g r$ is a limit of the corresponding $\widetilde{\mathcal{H}}$, where the PBW Theorem was already checked. We 
actually repeat here the deduction of the linear independence of the elements from $(i, i i)$ from the fact that nil-DAHA are limits of the general DAHA.

Note that the $\mathcal{F}_{m}$ are finite-dimensional vector spaces in contrast to the ord-filtration for $\overline{\mathcal{H H}}$, where all such spaces are infinite dimensional since $\operatorname{ord}\left(X^{ \pm 1}\right)=0$.

\section{INDUCED REPRESENTATIONS}

4.1. Tilde-polynomial modules. Replacing $X^{ \pm 1}$ by $\widetilde{X}, \widetilde{X}^{\prime}$ significantly increases the list of modules of polynomial type.

4.1.1. Tilde-induction. Let us begin with $\widetilde{\mathcal{H}}_{\pi, \widetilde{X}}$. The tilde-polynomial representations for this algebra are

$$
\widetilde{\mathscr{X}}_{\pi, \tilde{X}}^{\epsilon, \pm} \stackrel{\text { def }}{=} \operatorname{Ind}_{\widetilde{\mathcal{H}}_{\tilde{X}}} \widetilde{\mathcal{H}}_{\pi, \widetilde{X}} \mathrm{C}_{\tilde{X}}^{\epsilon, \delta},
$$

where $\widetilde{\mathcal{H}}_{\widetilde{X}} \stackrel{\text { def }}{=}<T, \widetilde{X}, \widetilde{X}^{\prime}>$. Here $\mathrm{C}_{\widetilde{X}}^{\epsilon, \delta}$ for $\epsilon=0,1, \delta= \pm$ is the restriction of the one-dimensional representation of $\overline{\mathcal{H}}_{\tilde{X}} \stackrel{\text { def }}{=}<T, \widetilde{X}, \widetilde{X}^{\prime}, \widetilde{\pi}>$ defined as follows (cf. (3.5)):

$$
\begin{aligned}
& T^{\prime}(1)=\epsilon, T(1)=\epsilon-1, \tilde{\pi}(1)=\delta, \\
& \tilde{X}(1)=\delta \epsilon, \tilde{X}^{\prime}(1)=\delta(\epsilon-1) .
\end{aligned}
$$

Here and in the sequel, $\delta= \pm$ is understood as \pm 1 when applicable.

Theorem 4.1. For $\epsilon=0$, the natural map

$$
\widetilde{\mathscr{X}}_{\pi, \widetilde{X}}^{0, \pm} \rightarrow \overline{\mathscr{X}}_{\tilde{X}}^{0, \pm} \stackrel{\text { def }}{=} \operatorname{Ind} \overline{\mathcal{H}}_{\tilde{X}} \mathrm{C}_{\widetilde{X}}^{0, \pm}
$$

is an isomorphism. Thus $\overline{\mathcal{H}}$ naturally acts in the former module. The counterpart of this claim for the algebra $\widetilde{\mathcal{H}}_{\tilde{\pi}, Y}$ (see 4.10 below) is for $\epsilon=1$ :

$$
\widetilde{\mathscr{X}}_{\widetilde{\pi}, Y}^{1, \pm} \rightarrow \overline{\mathscr{X}}_{Y}^{1, \pm} \stackrel{\text { def }}{=} \operatorname{Ind} \overline{\mathcal{H}}_{\overline{\mathcal{H}}_{Y}} \mathrm{C}_{\widetilde{X}}^{1, \pm}
$$

Proof. The first map is an embedding due to the PBW theorem. Thus we need only check that it is surjective. It suffices to check that the induced $\overline{\mathcal{H}}$-module $\overline{\mathscr{X}}_{\widetilde{X}}^{0, \pm}$ remains irreducible upon the restriction to $\widetilde{\mathcal{H}}_{\pi, \widetilde{X}}$ for generic $q$. Here we diagonalize $\widetilde{X}, \widetilde{X}^{\prime}$ in $\widetilde{\mathscr{X}}_{\widetilde{X}}^{0, \pm}$ and check that their spectrum is simple. This is completely analogous to the diagonalization of $Y, Y^{\prime}$ in the standard polynomial representation $\mathscr{X}$. Then we use that $\pi$ intertwines $\widetilde{X}$ and $\widetilde{X}^{\prime}$. 
The adjustment of $\epsilon$ when inducing from $Y, Y^{\prime}$ versus $\tilde{X}, \widetilde{X}^{\prime}$ really is necessary; see Proposition 4.4 below.

4.1.2. Explicit identification. Theorem 4.1 can be obtained directly via explicit formulas for the action of the generators, i.e., without any reference to the irreducibility of the induced polynomial-type representations for generic $q$. Later we will need the explicit formulas anyway.

Let us consider the induced modules governed by the PBW Theorem from $(i i)$ where we take $\epsilon=0, \delta= \pm$. There are five types of monomials $N$ listed in Theorem 3.4, $(i i)$. Only the following evaluations of such $N$ at $1 \in \widetilde{\mathscr{X}}_{\pi, \widetilde{X}}^{\epsilon, \delta}$ do not vanish:

$$
Y^{l} \pi\left(\tilde{X}^{\prime}\right)^{m}(1), Y^{l}(1)(l>0),\left(Y^{\prime}\right)^{l}\left(\tilde{X}^{\prime}\right)^{m}(1) .
$$

We use that $T^{\prime}(1)=0, \tilde{X}(1)=\tilde{\pi} T^{\prime}(1)=0$. Since $Y^{l}(1)=Y^{l-1} \pi T(1)=$ $-Y^{l-1} \pi$, the evaluations $Y^{l}(1)$ with $l>0$ can be omitted (this case is included in the previous one). Moreover, the relation $T(1)=-1$ results in

$$
\pi(1)=-\pi T(1)=-Y(1), Y^{l} \pi\left(\widetilde{X}^{\prime}\right)^{m}(1)=-(-\delta)^{m} Y^{l+1}(1) .
$$

We see that the evaluation at 1 naturally leads to the identification

$$
\widetilde{\mathscr{X}_{\pi, \widetilde{X}}^{0, \delta}} \cong\left\{\sum_{l \geq 0} a_{l+1} Y^{l+1}(1)+a_{-l}\left(Y^{\prime}\right)^{l}(1)\right\}
$$

the coefficients are from $\mathrm{C}\left[q^{ \pm 1 / 4}\right]$. To prove the proposition in these two cases $(\delta= \pm)$, we need to check that the latter space is invariant under the application of $\tilde{\pi}$, which is missing in $\widetilde{\mathcal{H}}_{\pi, \widetilde{X}}$. Recall that $\tilde{\pi}(1)=\delta= \pm 1 ;$ see $(4.1)$.

Using the relation $\tilde{\pi} Y \tilde{\pi}=q^{-1 / 2} Y^{\prime}$ from (3.31),

$$
\begin{aligned}
& \tilde{\pi}\left(\sum_{l \geq 0} a_{l+1} Y^{l+1}+a_{-l}\left(Y^{\prime}\right)^{l}\right)(1) \\
& \quad=\delta\left(\sum_{l \geq 0} a_{l+1} q^{-(l+1) / 2}\left(Y^{\prime}\right)^{l+1}+a_{-l} q^{l / 2} Y^{l}\right)(1)
\end{aligned}
$$

which gives the required "extra" formula.

For $\widetilde{\mathscr{X}}_{\widetilde{\pi}, Y}^{1, \pm}$, the demonstration of Theorem 4.1 via the explicit formulas for $\pi$, missing in $\widetilde{\mathcal{H}}_{\widetilde{\pi}, Y}$, is analogous. Note that we take $\epsilon=1$ in this case; see Proposition 4.4 below. 
4.1.3. Basic operators. Continuing our explicit analysis based on the identification from (4.3), let us obtain the formulas for the action of $T, \pi$ in this tilde-polynomial module (which are guaranteed by the induction construction).

Generalizing relations $T Y^{\prime}=Y T^{\prime}, Y^{\prime} T=T^{\prime} Y$ and using that $Y Y^{\prime}=$ $Y^{\prime} Y$, we arrive at $T\left(Y^{\prime}\right)^{2}=Y T^{\prime} Y^{\prime}=Y(T+1) Y^{\prime}=Y^{2} T^{\prime}, \ldots$,

$$
T\left(Y^{\prime}\right)^{m}=Y^{m} T^{\prime},\left(Y^{\prime}\right)^{m} T=T^{\prime} Y^{m} \text { for } m>0 .
$$

Thus,

$$
\begin{aligned}
& T\left(\sum_{l \geq 0} a_{l+1} Y^{l+1}+a_{-l}\left(Y^{\prime}\right)^{l}\right)(1) \\
& \quad=\sum_{l \geq 0}-a_{l+1}\left(Y^{l+1}+\left(Y^{\prime}\right)^{l+1}\right)(1) .
\end{aligned}
$$

Similarly, $\pi Y=T$ and $\pi\left(Y^{m+1}\right)=\pi Y^{m+1}(1)=T\left(Y^{m}\right)$ for $m \geq 0$. Using that $\pi Y^{\prime}=\pi T^{\prime} \pi=Y \pi+1$,

$$
\begin{aligned}
\pi\left(Y^{\prime}\right)^{2}=(Y \pi+1) Y^{\prime} & =Y(Y \pi+1)+Y^{\prime}=Y^{2} \pi+Y+Y^{\prime}, \quad \pi\left(Y^{\prime}\right)^{3}= \\
=\left(Y^{2} \pi+Y+Y^{\prime}\right) Y^{\prime} & =Y^{2}(Y \pi+1)+\left(Y^{\prime}\right)^{2}=Y^{3} \pi+Y^{2}+\left(Y^{\prime}\right)^{2}, \ldots, \\
& (4.7) \quad \pi\left(Y^{\prime}\right)^{m}=Y^{m} \pi+Y^{m-1}+\left(Y^{\prime}\right)^{m-1} \text { for } m>1 .
\end{aligned}
$$

Due to $\pi(1)=-Y(1)=-Y$, we obtain finally that

$$
\begin{aligned}
\pi & \left(\sum_{l \geq 0} a_{l+1} Y^{l+1}+a_{-l}\left(Y^{\prime}\right)^{l}\right)(1) \\
& =\sum_{l \geq 0}-a_{l+1} M_{l}(1)+\sum_{l \geq 0}-a_{-l}\left(Y^{l+1}-M_{l-1}\right)(1),
\end{aligned}
$$

where $M_{m}=Y^{m}+\left(Y^{\prime}\right)^{m}$ for $m>0, M_{0}=1, M_{-1}=0$.

This provides a complete and effective description of the action of $\overline{\mathcal{H}}$ in the tilde-polynomial representation $\widetilde{\mathscr{X}}_{\pi, \widetilde{X}}^{0, \delta}$ upon the identification from (4.3). Indeed, $Y, Y^{\prime}$ act by the multiplication and the action of $X, X^{-1}$ can be calculated using $(4.4,4.7)$ and $X=q^{1 / 4} \tilde{\pi} \pi$. One has:

$$
\begin{aligned}
& X(1)=-\delta q^{-\frac{1}{4}} Y^{\prime}(1)=-\delta q^{-\frac{1}{4}} Y^{\prime}, X(Y)(1)=-\delta q^{\frac{1}{4}}, \\
& X\left(Y^{\prime}\right)(1)=-\delta q^{\frac{1}{4}}\left(q^{-1}\left(Y^{\prime}\right)^{2}-1\right)(1)=\delta q^{\frac{1}{4}}\left(q^{-1}\left(Y^{\prime}\right)^{2}-1\right) .
\end{aligned}
$$

and for $m \geq 2$ :

$$
X\left(Y^{m}\right)(1)=-\delta q^{\frac{1}{4}}\left(q^{\frac{m-1}{2}} Y^{m-1}+q^{-\frac{m-1}{2}}\left(Y^{\prime}\right)^{m-1}\right)(1),
$$

$$
X\left(Y^{\prime}\right)^{m}(1)=-\delta q^{\frac{1}{4}}\left(q^{-\frac{m+1}{2}}\left(Y^{\prime}\right)^{m+1}-q^{\frac{m-1}{2}} Y^{m-1}-q^{-\frac{m-1}{2}}\left(Y^{\prime}\right)^{m-1}\right)(1) .
$$

These formulas can be identified with those for the action of the spinorDunkl operators in the spinor representation; see (5.12) below. 
Let us briefly consider the subalgebra $\widetilde{\mathcal{H}}_{\widetilde{\pi}, Y}$ following part $(i)$ of Theorem 3.4. The tilde-polynomial representations for this algebra are

$$
\widetilde{\mathscr{X}}_{\tilde{\pi}, Y}^{1, \pm} \stackrel{\text { def }}{=} \operatorname{Ind}_{\widetilde{\mathcal{H}}_{Y}}^{\widetilde{\mathcal{H}}_{\tilde{\pi}, Y}} \mathrm{C}_{Y}^{1, \pm}
$$

where $\widetilde{\mathcal{H}}_{Y} \stackrel{\text { def }}{=}<T, Y, Y^{\prime}>$. Analogous to $(i i), \mathrm{C}_{Y}^{\epsilon, \delta}$ is the restriction of the one-dimensional representations of $\overline{\mathcal{H}}_{Y, \pi} \stackrel{\text { def }}{=}<T, Y, Y^{\prime}, \pi>$ given by

$$
\begin{aligned}
& T^{\prime}(1)=\epsilon, T(1)=\epsilon-1, \pi(1)=\delta, \\
& Y(1)=\delta(\epsilon-1), Y^{\prime}(1)=\delta \epsilon, \epsilon=0,1, \delta= \pm .
\end{aligned}
$$

to its subalgebra $\overline{\mathcal{H}}_{Y} \stackrel{\text { def }}{=}<T, Y, Y^{\prime}>$.

Now $\epsilon=1$, which gives that $T(1)=0=Y(1), \pi(1)=\delta=Y(1)$ and $\tilde{\pi}(1)=\widetilde{X}(1)$ due to $T^{\prime}(1)=1$. Since we induce from the same $\overline{\mathcal{H}}_{Y, \pi}$ as for the polynomial representation, the resulting tilde-representation canonically coincides with $\overline{\mathscr{X}}=\mathrm{C}\left[q^{ \pm 1 / 4}\right]\left[X^{ \pm 1}\right]$.

Proposition 4.2. The polynomial representation $\overline{\mathscr{X}}$ can be naturally identified with

$$
\widetilde{\mathscr{X}_{\widetilde{\pi}, Y}^{1,+}} \cong\left\{\sum_{l \geq 0} a_{l+1} \widetilde{X}^{l+1}(1)+a_{-l}\left(\widetilde{X}^{\prime}\right)^{l}(1)\right\}
$$

for the action of the powers of $\widetilde{X}, \widetilde{X}^{\prime}$ on 1 in the tilde-polynomial representation. Equivalently, $\left\{\tilde{X}^{l+1}(1),\left(\tilde{X}^{\prime}\right)^{l}(1), l \geq 0\right\}$ is a basis of $\overline{\mathscr{X}}$, where $H(1)$ is understood here as $H$ applied to 1 in $\overline{\mathscr{X}}$.

The final form of this proposition is presented in Theorem 4.8 below.

4.2. The core subalgebra. It is now quite natural to consider the intersection subalgebra

$$
\widetilde{\mathcal{H}}_{Y, \widetilde{X}} \stackrel{\text { def }}{=} \widetilde{\mathcal{H}}_{\widetilde{\pi}, Y} \cap \widetilde{\mathcal{H}}_{\pi, \widetilde{X}}
$$

By construction, this subalgebra is preserved by the anti-involution $\psi$ from (3.35) sending

$$
Y \mapsto \tilde{X}^{\prime}, Y^{\prime} \mapsto \tilde{X}, \widetilde{\pi} \mapsto \pi, T \mapsto T .
$$


Theorem 4.3. (i) (Defining relations) As an abstract algebra, $\widetilde{\mathcal{H}}_{Y, \tilde{X}}$ is generated by $\widetilde{X}, \widetilde{X}^{\prime}, Y, Y^{\prime}, T$ subject to the relations

$$
\begin{aligned}
& T T^{\prime}=0=T^{\prime} T=\widetilde{X} \widetilde{X}^{\prime}=\widetilde{X}^{\prime} \widetilde{X}=Y Y^{\prime}=Y^{\prime} Y, \\
& \tilde{X} T=0=T^{\prime} \tilde{X}^{\prime}=Y T^{\prime}=T Y^{\prime}=\tilde{X}^{\prime} Y=Y^{\prime} \widetilde{X}, \\
& T \tilde{X}=\tilde{X}^{\prime} T^{\prime}, \quad T^{\prime} Y=Y^{\prime} T, \quad \tilde{X} Y^{\prime}=-q^{1 / 2} Y \tilde{X}^{\prime}, \\
& \widetilde{X} Y=q^{1 / 2} Y\left(\tilde{X}+\tilde{X}^{\prime}\right), \quad \tilde{X}^{\prime} Y^{\prime}=q^{1 / 2}\left(Y^{\prime}+Y\right) \tilde{X}^{\prime} .
\end{aligned}
$$

(ii) (PBW Theorem) An arbitrary element $H \in \widetilde{\mathcal{H}}_{Y, \widetilde{X}}$ can be uniquely expressed as a linear combination of the elements

$$
\begin{aligned}
& Y^{l} \tilde{X}^{m}, \quad\left(Y^{\prime}\right)^{l}\left(\tilde{X}^{\prime}\right)^{m}(l+m>0), \\
& Y^{l}\left(\tilde{X}^{\prime}\right)^{m}(l m>0), \quad\left(Y^{\prime}\right)^{l}\left(\tilde{X}^{\prime}\right)^{m} T .
\end{aligned}
$$

Here the ordering $\left\{Y, Y^{\prime}\right\},\left\{\widetilde{X}, \widetilde{X}^{\prime}\right\}, T$ can be changed to its arbitrary permutation (similar to the $q, t$-case).

(iii) (Tilde-Weyl algebra) For the order from Proposition 3.5 restricted to $\widetilde{\mathcal{H}}_{Y, \widetilde{X}}$ the corresponding gr $\widetilde{\mathcal{H}}_{Y, \widetilde{X}}$ is algebraically generated by $\mathrm{C}$ and the images of following elements in $\widetilde{\mathcal{H}}_{Y, \widetilde{X}}$ of order one

$$
\widetilde{X}, \tilde{X}^{\prime}, Y, Y^{\prime}, T, P \stackrel{\text { def }}{=} \widetilde{X} Y^{\prime}=-q^{1 / 2} Y \widetilde{X}^{\prime}
$$

subject to the nil-nil-Weyl relations:

$$
\begin{aligned}
& \widetilde{X} \tilde{X}^{\prime}=Y Y^{\prime}=0=\tilde{X}^{\prime} \widetilde{X}=Y^{\prime} Y, T \widetilde{X}=\widetilde{X}^{\prime} T, T Y=Y^{\prime} T, \\
& T^{2}=0=\tilde{X} T=T \widetilde{X}^{\prime}=Y T=T Y^{\prime}=\widetilde{X}^{\prime} Y=Y^{\prime} \widetilde{X}, \\
& P^{2}=0=P \widetilde{X}=\tilde{X}^{\prime} P=P Y=Y^{\prime} P=\widetilde{X} Y^{\prime}=Y \tilde{X}^{\prime}, \\
& T P=\tilde{X}^{\prime} Y^{\prime}, \quad P T=\tilde{X} Y, \quad P Y^{\prime}=q^{1 / 2} Y P, \quad \widetilde{X} P=q^{1 / 2} P \tilde{X}^{\prime},
\end{aligned}
$$

$$
\tilde{X} Y=q^{1 / 2} Y \tilde{X}, \quad \tilde{X}^{\prime} Y^{\prime}=q^{1 / 2} Y^{\prime} \tilde{X}^{\prime}
$$

(iv) (Induced modules) Setting

$$
\begin{aligned}
& \widetilde{\mathscr{X}}_{Y}^{\epsilon, \pm} \stackrel{\text { def }}{=} \operatorname{Ind}_{\widetilde{\mathcal{H}}_{Y, \widetilde{X}}} \mathrm{C}_{Y}^{\epsilon, \pm}, \\
& \widetilde{\mathscr{X}}_{\widetilde{X}}^{\epsilon, \pm} \stackrel{\text { def }}{=} \operatorname{Ind}_{\widetilde{\mathcal{H}}_{\tilde{X}}}^{\widetilde{\mathcal{H}}_{Y, \widetilde{X}}} \mathrm{C}_{\widetilde{X}}^{\epsilon, \pm}
\end{aligned}
$$

for $\mathrm{C}^{\epsilon, \pm}$ defined in $(3.5,4.1)$, the natural maps

$$
\widetilde{\mathscr{X}_{Y}^{1, \delta}} \rightarrow \widetilde{\mathscr{X}_{\widetilde{\pi}, Y}^{1, \delta}}, \quad \widetilde{\mathscr{X}_{\widetilde{X}}^{0, \delta}} \rightarrow \widetilde{\mathscr{X}_{\pi, \widetilde{X}}^{0, \delta}}
$$


are isomorphisms for $\delta= \pm$.

Proof. The vanishing conditions for the monomials from part $(i)$ are due to $T T^{\prime}=0=T^{\prime} T$ directly or (less directly) as in (3.41) and (3.42). Next, the relations

$$
\begin{aligned}
& Y \tilde{X}^{\prime}=\pi T^{2} \widetilde{\pi}=-\pi T \widetilde{\pi} \\
= & -Y \widetilde{\pi}=-q^{-1 / 2} \tilde{\pi} Y^{\prime}=-\pi \widetilde{X}^{\prime}=-q^{-1 / 2} \widetilde{X} \pi,
\end{aligned}
$$

combined with the analogous ones

$$
\widetilde{X} Y^{\prime}=\tilde{\pi} T^{\prime} \pi=\widetilde{X} \pi=\tilde{\pi} Y^{\prime},
$$

readily result in

$$
Y \widetilde{X}^{\prime}=-q^{-1 / 2} \tilde{X} \pi=-q^{-1 / 2} \tilde{X} Y^{\prime}
$$

Now,

$$
\begin{aligned}
& \tilde{X} Y=\tilde{\pi} T^{\prime} Y=\tilde{\pi} Y^{\prime} T=q^{1 / 2} Y \widetilde{\pi} T=q^{1 / 2} Y \widetilde{\pi}\left(T^{\prime}-1\right) \\
& =q^{1 / 2} Y \widetilde{X}-q^{1 / 2} Y \widetilde{\pi}=q^{1 / 2} Y\left(\widetilde{X}+\widetilde{X}^{\prime}\right)
\end{aligned}
$$

where we use (4.19) and $\tilde{\pi} Y^{\prime} \tilde{\pi}=q^{1 / 2} Y$ from (3.38). Similarly,

$$
\begin{aligned}
& \tilde{X}^{\prime} Y^{\prime}=T \widetilde{\pi} Y^{\prime}=q^{1 / 2} T Y \widetilde{\pi}=q^{1 / 2}\left(T^{\prime}-1\right) Y \widetilde{\pi} \\
& =q^{1 / 2} Y^{\prime} T \widetilde{\pi}-q^{1 / 2} Y \widetilde{\pi}=q^{1 / 2} Y^{\prime} \widetilde{X}^{\prime}-q^{1 / 2} Y \widetilde{\pi}=q^{1 / 2}\left(Y^{\prime}+Y\right) \widetilde{X}^{\prime} .
\end{aligned}
$$

We see that the relations from $(i)$ hold in $\overline{\mathcal{H}}$. These relations are sufficient to ensure that the elements listed in (ii) linearly generate $\widetilde{\mathcal{H}}_{Y, \widetilde{X}}$, which is sufficient to establish their linear independence.

The passage to $g r$ is straightforward; express $P$ using relations (4.19)

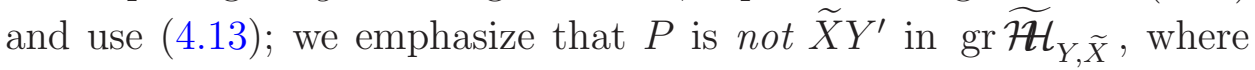
$\tilde{X} Y^{\prime}=0$. Since $P$ is $\psi$-invariant, the following are sufficient to check the relations where $P$ appears:

$$
\begin{aligned}
& T P=T \widetilde{X} Y^{\prime}=\widetilde{X}^{\prime}\left(T^{\prime}\right)^{2} Y^{\prime}=\tilde{X}^{\prime} T^{\prime} Y^{\prime}=\widetilde{X}^{\prime} Y^{\prime} \text { and } \\
& \left.\left(Y \widetilde{X}^{\prime}\right) Y^{\prime}=q^{1 / 2} Y\left(Y^{\prime} \widetilde{X}^{\prime}+Y \tilde{X}\right)=q^{1 / 2} Y^{2} \widetilde{X}\right)=q^{1 / 2} Y\left(Y \widetilde{X}^{\prime}\right) .
\end{aligned}
$$

Both identities hold in $\widetilde{\mathcal{H}}_{\widetilde{\pi}, Y}$, i.e., before taking $g r$.

Finally, the claims from $(i v)$ follow from $(i, i i)$ and the previous considerations for $\widetilde{\mathcal{H}}_{\widetilde{\pi}, Y}$ and $\widetilde{\mathcal{H}}_{\pi, \widetilde{X}}$.

The last part of the theorem is the most logically transparent way to introduce the tilde-polynomial representations. The intermediate subalgebras $\widetilde{\mathcal{H}}_{\widetilde{\pi}, Y}$ and $\widetilde{\mathcal{H}}_{\pi, \widetilde{X}}$ are transitional, as they are convenient for calculating the formulas for the action of $\pi, \widetilde{\pi}$ and $X^{ \pm 1}$. 
We note that one can introduce a different order $\widetilde{\text { ord }}$ in $\widetilde{\mathcal{H}}_{Y, \widetilde{X}}$, not the order ord from $\widetilde{\mathcal{H}}$ used in $($ iii $)$, by making $\widetilde{\operatorname{ord}}(P)=2$. Namely, the elements of tilde-order no greater than 1 will be $1, T, Y, Y^{\prime}, \widetilde{X}, \widetilde{X^{\prime}}$ and their linear combinations. They will algebraically generate $\widetilde{g r} \widetilde{\mathcal{H}}{ }_{Y, \widetilde{X}}$ subject to the relations from $(i)$ where we set $T^{2}=0$ and replace $T^{\prime}$ everywhere by $T$. We use that these relations are homogeneous for $\widetilde{\text { ord }}$ upon the formal identification of $T$ and $T^{\prime}$; for instance, the relations

$$
\tilde{X} Y=q^{1 / 2} Y\left(\tilde{X}+\tilde{X}^{\prime}\right), \quad \tilde{X}^{\prime} Y^{\prime}=q^{1 / 2}\left(Y^{\prime}+Y\right) \tilde{X}^{\prime}
$$

remain unchanged after taking $\widetilde{g r}$. Such graded algebra does not seem (immediately) connected with the "natural" nil-nil-Weyl algebra from (iii) (which can be generalized to higher ranks).

In contrast to the intermediate subalgebras and the main nil-DAHA $\overline{\mathcal{H}}$, the algebra $\widetilde{\mathcal{H}}_{Y, \widetilde{X}}$ is bi-graded. We set

$$
\begin{gathered}
\operatorname{deg}_{\widetilde{x}, y}(T)=0, \operatorname{deg}_{\widetilde{x}, y}(A B)=\operatorname{deg}_{\widetilde{x}, y}(A)+\operatorname{deg}_{\widetilde{x}, y}(B), \\
\operatorname{deg}_{y}\left(Y^{m}\right)=m=\operatorname{deg}_{y}\left(\left(Y^{\prime}\right)^{m}\right), \operatorname{deg}_{\widetilde{x}}\left(\widetilde{X}^{m}\right)=m=\operatorname{deg}_{\widetilde{x}}\left(\left(\widetilde{X}^{\prime}\right)^{m}\right), \\
\operatorname{deg}_{y}\left(\widetilde{X}^{m}\right)=0=\operatorname{deg}_{y}\left(\left(\widetilde{X}^{\prime}\right)^{m}\right), \operatorname{deg}_{\widetilde{x}}\left(Y^{m}\right)=0=\operatorname{deg}_{\widetilde{x}}\left(\left(Y^{\prime}\right)^{m}\right),
\end{gathered}
$$

where $A, B \in \widetilde{\mathcal{H}}_{Y, \widetilde{X}} ; q$ is of degree zero.

Note that the subalgebras $\widetilde{\mathcal{H}}_{\widetilde{\pi}, Y}$ and $\widetilde{\mathcal{H}}_{\pi, \tilde{X}}$ possess $\operatorname{deg}_{y}$ and $\operatorname{deg}_{\widetilde{x}}$, respectively (but not both). This bi-grading plays a significantly more important role in the paper than the order in (iii).

4.2.1. Tilde-intertwiners. The modules from (4.17) are graded and even bi-graded upon the following modification of their definitions. Let $\widetilde{\mathscr{X}}_{\widetilde{X}}^{\epsilon, u}$ be induced from the one-dimensional $\widetilde{\mathcal{H}}_{\widetilde{X}}$-modules $\mathrm{C}_{\widetilde{X}}^{\epsilon, u}$ introduced as follows:

$$
\begin{aligned}
& \tilde{X}(1)=0=T^{\prime}(1), \widetilde{X}^{\prime}(1)=-u \text { for } \epsilon=0, \\
& \tilde{X}^{\prime}(1)=0=T(1), \tilde{X}(1)=u \text { for } \epsilon=1 .
\end{aligned}
$$

The degree $\operatorname{deg}_{y}$ is present here. If $u$ is considered an independent variable such that $\operatorname{deg}_{\widetilde{x}}(u)=1$, then the latter degree can be extended to $\widetilde{\mathscr{X}_{\widetilde{X}}^{\epsilon}}, u$. Such bi-grading will not be used in the paper; we will mainly treat $u$ as a constant. However, $\operatorname{deg}_{y}$ will be needed.

Analogously, the $\widetilde{\mathcal{H}}_{Y}-$ modules $\mathrm{C}_{Y}^{\epsilon, u}$ are

$$
\begin{aligned}
& Y^{\prime}(1)=0=T^{\prime}(1), Y(1)=-u \text { for } \epsilon=0, \\
& Y(1)=0=T(1), Y^{\prime}(1)=u \text { for } \epsilon=1 .
\end{aligned}
$$


The corresponding induced modules are denoted by $\widetilde{\mathscr{X}}_{Y}^{\epsilon, u}$; the grading here is $\operatorname{deg}_{\widetilde{x}}$.

These starting one-dimensional modules can be naturally extended to one-dimensional $\widetilde{\mathcal{H}}_{Y, \widetilde{X}}-$ modules, which will be denoted by $\widetilde{\mathrm{C}}_{\widetilde{X}}^{\epsilon, u}$ and $\widetilde{\mathrm{C}}_{Y}^{\epsilon, u}$. The action of the remaining operators is zero, namely, $Y(1)=$ $0=Y^{\prime}(1)$ for $(4.21)$ and $\widetilde{X}(1)=0=\widetilde{X}^{\prime}(1)$ for $(4.22)$.

The following proposition explains why only one choice of $\epsilon$ is possible for each type of the induction; see Theorem 4.3, (iv).

Proposition 4.4. (i) The modules

$$
\widetilde{\mathscr{X}_{Y}^{0, u}} \text { and } \widetilde{\mathscr{X}_{\widetilde{X}}^{1, u}}
$$

are one-dimensional. They are equal to $\widetilde{\mathrm{C}}_{\widetilde{X}}^{0, u}$ and $\widetilde{\mathrm{C}}_{Y}^{1, u}$, respectively.

(ii) Assuming that $q$ is generic (i.e., not a root of unity), the only nonzero irreducible finite-dimensional $\widetilde{\mathcal{H}}_{Y, \widetilde{X}}-$ modules are:

$$
\widetilde{\mathrm{C}}_{Y}^{1, u}, \widetilde{\mathrm{C}}_{Y}^{0, u} \text { or } \widetilde{\mathrm{C}}_{\widetilde{X}}^{1, u}, \widetilde{\mathrm{C}}_{\widetilde{X}}^{0, u} \text { for } u \neq 0 \text {. }
$$

Proof. Let us start with $\widetilde{\mathscr{X}}_{\widetilde{X}}^{1, u}$. Since $\epsilon=1$,

$$
T^{\prime}(1)=1, T(1)=0=\widetilde{X}^{\prime}(1), \widetilde{X}(1)=u .
$$

Using that $Y^{\prime} \widetilde{X}=0$, one of the defining relations from part $(i)$ of the theorem, we obtain that $Y^{\prime}(1)=0$. Also, $Y T^{\prime}=0$, which gives that $Y(1)=Y T^{\prime}(1)=0$. Thus the induced module is one-dimensional in this case, where only $\widetilde{X}$ and $T^{\prime}$ are non-zero among $Y, Y^{\prime}, \widetilde{X}, \widetilde{X}^{\prime}, T, T^{\prime}$.

Similarly, if $\epsilon=0$ and we induce from $Y, Y^{\prime}$, then:

$$
T^{\prime}(1)=0, T(1)=-1, Y^{\prime}(1)=0, Y(1)=-u,
$$

which results in $\widetilde{X}(1)=0$ due to $\widetilde{X} T=0$ and in $\widetilde{X}^{\prime}(1)=0$ due to $\widetilde{X}^{\prime} Y=0$. Part $(i)$ is verified.

Lemma 4.5. (i) Let us assume that $Y(e)=\Lambda e$ for $\Lambda \neq 0$. Then $Y^{\prime}(e)=0=\widetilde{X}^{\prime}(e)$. If $\widetilde{e} \stackrel{\text { def }}{=} \widetilde{X}(e) \neq 0$, then

$$
Y(\widetilde{e})=q^{1 / 2} \Lambda \widetilde{e}, Y^{\prime}(\widetilde{e})=0=\widetilde{X}^{\prime}(\widetilde{e}) .
$$

Let $e^{\prime} \stackrel{\text { def }}{=} T^{\prime}(e) \neq 0$. Then $Y^{\prime}\left(e^{\prime}\right)=\Lambda e^{\prime}, Y\left(e^{\prime}\right)=0=T\left(e^{\prime}\right)$. If $T^{\prime}(e)=0$ then $\widetilde{X}(e)=0$ and the module $\widetilde{\mathcal{H}}_{Y, \widetilde{X}}(e)$ equals $\widetilde{\mathrm{C}}_{Y}^{0, u}$ for $u=-\Lambda^{\prime}$. 
(ii) Now we assume that $Y^{\prime}\left(e^{\prime}\right)=\Lambda^{\prime} e^{\prime}$ for $\Lambda^{\prime} \neq 0$, which automatically results in $Y\left(e^{\prime}\right)=0=T\left(e^{\prime}\right)$, and set $\widetilde{e}^{\prime} \stackrel{\text { def }}{=} \widetilde{X}\left(e^{\prime}\right)$. Then

$$
Y(\widetilde{e})=q^{-1 / 2} \Lambda^{\prime} \widetilde{e}, Y^{\prime}\left(\widetilde{e}^{\prime}\right)=0=\widetilde{X}^{\prime}\left(\widetilde{e}^{\prime}\right) .
$$

If here $\widetilde{e}=0$, then $\widetilde{X}^{\prime}\left(e^{\prime}\right)=0$ and the module $\widetilde{\mathcal{H}}_{Y, \widetilde{X}}\left(e^{\prime}\right)$ equals $\widetilde{\mathrm{C}}_{Y}^{1, u}$ for $u=q^{-1 / 2} \Lambda^{\prime}$.

(iii) The statements from (i) hold correspondingly for $\widetilde{e} \stackrel{\text { def }}{=} Y(e)$ and $e^{\prime} \stackrel{\text { def }}{=} T(e)$ assuming that $\tilde{X}(e)=\Lambda e$ for $\Lambda \neq 0$; the latter implies that $Y^{\prime}(e)=0=\tilde{X}^{\prime}(e)$. In this case,

$$
\widetilde{X}(\widetilde{e})=q^{1 / 2} \Lambda \widetilde{e}, \widetilde{X}^{\prime}\left(e^{\prime}\right)=\Lambda e^{\prime} ; T(e)=0 \Rightarrow Y(e)=0 .
$$

Following (ii), let $\widetilde{X}^{\prime}\left(e^{\prime}\right)=\Lambda^{\prime} e^{\prime}$ for $\Lambda^{\prime} \neq 0$ and $\widetilde{e} \stackrel{\text { def }}{=} Y\left(e^{\prime}\right)$. Then $\widetilde{X}\left(\widetilde{e}^{\prime}\right)=q^{-1 / 2} \Lambda^{\prime} \widetilde{e}^{\prime}$.

Proof. First of all, $Y^{\prime} Y(e)=0=\Lambda Y^{\prime}(e)$ and $\tilde{X}^{\prime}(e)=0$ due to the identity $\widetilde{X}^{\prime} Y=0$. For $\widetilde{e} \stackrel{\text { def }}{=} \widetilde{X}(e)$,

$$
Y(\widetilde{e})=Y \widetilde{X}(e)=\left(q^{1 / 2} \widetilde{X} Y-Y \widetilde{X}^{\prime}\right)(e)=q^{1 / 2} \widetilde{X} Y(e)=q^{1 / 2} \Lambda \widetilde{e} .
$$

Then automatically, $Y^{\prime}(\widetilde{e})=0=\widetilde{X}^{\prime}(\widetilde{e})$.

Setting $e^{\prime} \stackrel{\text { def }}{=} T^{\prime}(e)$, we arrive at:

$$
Y^{\prime}\left(e^{\prime}\right)=Y^{\prime} T^{\prime}(e)=Y^{\prime}(T+1)(e)=T^{\prime} Y(e)=\Lambda e^{\prime}, Y\left(e^{\prime}\right)=0=T\left(e^{\prime}\right) .
$$

Now, if $T^{\prime}(e)=0$ then $0=\tilde{X} T(e)=-\tilde{X}(e)$, since $\tilde{X} T=0$ and $T(e)=-e$.

(ii) One has $Y Y^{\prime}\left(e^{\prime}\right)=0=\Lambda^{\prime} Y(e)$ and $T Y^{\prime}\left(e^{\prime}\right)=\Lambda^{\prime} T\left(e^{\prime}\right)=$ $Y T^{\prime}\left(e^{\prime}\right)=0$. Using (4.13) from Theorem 4.3,

$$
\begin{aligned}
& Y \widetilde{X}^{\prime}\left(e^{\prime}\right)=-q^{-1 / 2} \widetilde{X} Y^{\prime}\left(e^{\prime}\right)=-q^{-1 / 2} \Lambda^{\prime} \widetilde{X}\left(e^{\prime}\right)=-q^{-1 / 2} \Lambda^{\prime} \widetilde{e}^{\prime} \\
& Y \widetilde{X}^{\prime}\left(e^{\prime}\right)=q^{1 / 2} \widetilde{X} Y\left(e^{\prime}\right)-Y \widetilde{X}\left(e^{\prime}\right)=0-Y \widetilde{X}\left(e^{\prime}\right)=-Y\left(\widetilde{e}^{\prime}\right) .
\end{aligned}
$$

If $\widetilde{e}^{\prime}=0$ then $\widetilde{X}\left(e^{\prime}\right)=0$, which results in

$$
\widetilde{X}^{\prime} T^{\prime}\left(e^{\prime}\right)=0=\widetilde{X}^{\prime}\left(e^{\prime}\right) \text { due to } T^{\prime}\left(e^{\prime}\right)=e^{\prime} .
$$

Note that $\tilde{X}=\tilde{\pi} T^{\prime}$ is proportional to the product $\Pi(T+1)$ of the intertwining operators used in (2.35). Thus, this claim and those from ( $i$ ) formalize the technique of intertwiners in the tilde-setting.

The counterparts of $(i)$ and $(i i)$ for $\widetilde{X}$ instead of $Y$ are straightforward. Let us demonstrate that $\widetilde{X}\left(\widetilde{e}^{\prime}\right)=q^{-1 / 2} \Lambda^{\prime} \widetilde{e}$ if for $\widetilde{e}=Y\left(e^{\prime}\right)$ if 
$\tilde{X}^{\prime}\left(e^{\prime}\right)=\Lambda^{\prime} e^{\prime}$. We need the following straightforward corollaries of the defining relation from $(4.13)$ :

$$
\widetilde{X} Y=q^{1 / 2} Y \tilde{X}-\tilde{X} Y^{\prime}, Y \tilde{X}=q^{-1 / 2} \tilde{X}\left(Y+Y^{\prime}\right) .
$$

Then, following (4.23),

$$
\begin{aligned}
& \widetilde{X} Y^{\prime}\left(e^{\prime}\right)=-q^{1 / 2} Y \widetilde{X}^{\prime}\left(e^{\prime}\right)=-q^{1 / 2} \Lambda^{\prime} Y\left(e^{\prime}\right)=-q^{1 / 2} \Lambda^{\prime} \widetilde{e}, \\
& \widetilde{X} Y^{\prime}\left(e^{\prime}\right)=q^{1 / 2} Y \widetilde{X}\left(e^{\prime}\right)-\widetilde{X} Y\left(e^{\prime}\right)=-\widetilde{X} Y\left(e^{\prime}\right)=-\widetilde{X}(\widetilde{e})
\end{aligned}
$$

and we can equate the outputs. Similarly, if $\widetilde{X}(e)=\Lambda e$ for $\Lambda \neq 0$, then $\tilde{X}^{\prime}(e)=0=Y^{\prime}(e)$ and $(4.24)$ gives that

$$
\widetilde{X}(\widetilde{e})=\widetilde{X} Y(e)=q^{1 / 2} Y \widetilde{X}(e)-\widetilde{X} Y^{\prime}(e)=q^{1 / 2} \Lambda \widetilde{e} .
$$

The lemma readily results in the description of the finite-dimensional irreducible representations from Proposition 4.4 (ii). Applying the intertwiners, we will eventually make $e^{\prime}=0$ or $\widetilde{e}=0$, provided $q$ is not a root of unity. Thus these modules can be only as claimed.

4.2.2. Dual induced modules. Since the induced modules from part $(i)$ of Proposition 4.4 are trivial, another approach must be used for establishing a link to the $\overline{\mathcal{H}}$-modules

$$
\overline{\mathscr{X}}_{\tilde{X}}^{1, \delta}, \overline{\mathscr{X}}_{Y}^{0, \delta} \text {. }
$$

These modules are isomorphic to $\mathrm{C}\left[q^{1 / 4}\right]\left[X^{ \pm 1}\right]$ as linear spaces; cf. Theorem 4.1. However they cannot be defined directly within the tildetheory. One must proceed as follows.

We set $\operatorname{Hom}_{\operatorname{deg}}(\mathcal{V}) \stackrel{\text { def }}{=} \lim _{m \rightarrow \infty} \operatorname{Hom}\left(\mathcal{V} / \mathcal{V}_{m}, \mathrm{C}\right)$ for a vector space $\mathcal{V}$ with the filtration $\mathcal{V}=\mathcal{V}_{0} \supset \mathcal{V}_{1} \supset \mathcal{V}_{2} \supset \cdots$, corresponding to a given degree function: $\mathcal{V}_{m}=\{v \in \mathcal{V}: \operatorname{deg}(v) \geq m\}$. We set $\mathcal{V}_{m}^{\vee} \underline{\underline{\text { def }}}$ $\operatorname{Hom}\left(\mathcal{V} / \mathcal{V}_{m+1}\right.$, C $)$. We will use the inner product $\langle f, g\rangle^{\prime}$ defined in (3.9) with corresponding anti-involution $\psi$.

Proposition 4.6. Let us define the following $\widetilde{\mathcal{H}}_{Y, \widetilde{X}}-$ modules:

$$
\begin{aligned}
& \left(\widetilde{\mathscr{X}_{Y}^{\epsilon, u}}\right)^{\vee} \stackrel{\text { def }}{=} \operatorname{Hom}_{\operatorname{deg}_{\widetilde{x}}}\left(\operatorname{Ind}_{\widetilde{\mathcal{H}}_{Y}} \widetilde{\mathcal{H}}_{Y, \widetilde{X}} C_{Y}^{\epsilon, u}, \mathrm{C}_{q}\right) \text {, } \\
& \left(\widetilde{\mathscr{X}}_{\widetilde{X}}^{\epsilon, u}\right)^{\vee} \stackrel{\text { def }}{=} \operatorname{Hom}_{\operatorname{deg}_{y}}\left(\operatorname{Ind}_{\widetilde{\mathcal{H}}_{\tilde{X}}} \widetilde{\mathcal{H}}_{Y, \widetilde{X}} \mathrm{C}_{\widetilde{X}}^{\epsilon, u}, \mathrm{C}_{q}\right) \text {, }
\end{aligned}
$$

where the action of $\widetilde{\mathcal{H}}_{Y, \widetilde{X}}$ in these spaces is via the anti-involution $\psi$ : $H(f(P)) \stackrel{\text { def }}{=} f(\psi(H)(P)) ; \mathrm{C}_{q} \stackrel{\text { def }}{=} \mathrm{C}\left[q^{ \pm 1 / 4}\right]$. 
(i) Let $\mathcal{V}_{m}^{\vee}$ be $\left(\widetilde{\mathscr{X}_{Y}^{1}}\right)_{m}^{\vee}$ or $\left(\widetilde{\mathscr{X}_{\widetilde{X}}^{0, u}}\right)_{m}^{\vee}$ with respect to $\operatorname{deg}_{\widetilde{x}}$ or $d e g_{y}, \mathcal{V}^{\vee}$ their inductive limit. Then

$$
\begin{aligned}
& \mathcal{V}=\mathcal{V}_{0} \supset \mathcal{V}_{1} \supset \mathcal{V}_{2} \supset \mathcal{V}_{3} \supset \cdots \\
& \mathrm{C}_{q} \cong \mathcal{V}_{1}^{\vee} \subset \mathcal{V}_{2}^{\vee} \subset \mathcal{V}_{3}^{\vee} \cdots \subset \mathcal{V}^{\vee}
\end{aligned}
$$

are actually filtrations of $\widetilde{\mathcal{H}}_{Y, \widetilde{X}}$-modules.

(ii) The pairing $\langle f, g\rangle^{\prime}$ induces the embeddings

$$
\overline{\mathscr{X}}_{\widetilde{X}}^{1, \delta} \hookrightarrow \operatorname{Hom}\left(\overline{\mathscr{X}}_{Y}^{1, \delta}, \mathrm{C}_{q}\right), \overline{\mathscr{X}}_{Y}^{0, \delta} \hookrightarrow \operatorname{Hom}\left(\overline{\mathscr{X}}_{\widetilde{X}}^{0, \delta}, \mathrm{C}_{q}\right),
$$

where $\delta= \pm$, which result in the isomorphisms of $\widetilde{\mathcal{H}}_{Y, \widetilde{X}}-$ modules

$$
\left(\widetilde{\mathscr{X}_{Y}^{1, \delta}}\right)^{\vee} \cong \overline{\mathscr{X}}_{\widetilde{X}}^{1, \delta}, \quad\left(\widetilde{\mathscr{X}_{\widetilde{X}}^{0, \delta}}\right)^{\vee} \cong \overline{\mathscr{X}}_{Y}^{0, \delta} .
$$

Proof. It suffices to check that the original descending filtration

$$
\mathcal{V}=\mathcal{V}_{0} \supset \mathcal{V}_{1} \supset \mathcal{V}_{2} \supset \mathcal{V}_{3} \ldots
$$

is that in terms of $\widetilde{\mathcal{H}}_{Y, \widetilde{X}}$-modules; here $\mathcal{V}$ is equal to $\widetilde{\mathscr{X}}_{Y}^{1, u}$ or $\widetilde{\mathscr{X}}_{\widetilde{X}}^{0, u}$. This readily follows from the properties of $d e g_{y}$ and $d e g_{\widetilde{x}}$. For (ii), we need to check that the embeddings in (4.28) are compatible with the corresponding deg. It results from $(i i)$; see also Theorem 4.7 below.

4.2.3. Decomposition. The deg-filtrations in

$$
\widetilde{\mathscr{X}}_{Y}^{1, \delta}, \quad\left(\widetilde{\mathscr{X}_{Y}^{1, \delta}}\right)^{\vee}, \quad \widetilde{\mathscr{X}_{\widetilde{X}}^{0, \delta}}, \quad\left(\widetilde{\mathscr{X}}_{\widetilde{X}}^{0, \delta}\right)^{\vee}
$$

give partial decomposition of the $\overline{\mathcal{H}}$-modules $\overline{\mathscr{X}}_{\tilde{X}}^{\epsilon, \delta}$ and $\overline{\mathscr{X}}_{Y}^{\epsilon, \delta}$ upon the restriction to $\widetilde{\mathcal{H}}_{Y, \widetilde{X}}$.

Recall that these filtrations $\left\{\mathcal{V}_{m}(m \geq 0)\right\}$ and $\left\{\mathcal{V}_{m}^{\vee}(m \geq 1)\right\}$ are correspondingly either descending co-finite-dimensional for the induced modules in (4.31) or ascending finite-dimensional for their dual counterparts.

For instance, in the check-case, the modules $\mathcal{V}_{1}^{\vee}$ from the proposition are $\widetilde{\mathrm{C}}_{\widetilde{X}}^{1, \delta}$ or $\widetilde{\mathrm{C}}_{Y}^{0, \delta}$, i.e., they are one-dimensional. The quotients $\mathcal{V}_{m+1}^{\vee} / \mathcal{V}_{m}^{\vee}$ are two-dimensional for $m \geq 1$. Since the irreducible $\widetilde{\mathcal{H}}_{Y, \widetilde{X}}-$ modules are all one-dimensional by part ( $i i$ ) of Proposition 4.4, the constituents in this filtration are not irreducible. The (complete) Jordan-Hoelder filtration is actually a counterpart of the filtration in the polynomial representation arising from the construction of nonsymmetric Macdonald polynomials. 
We use the identifications $A \mapsto A(1)$ :

$$
\widetilde{\mathscr{X}}_{Y}^{1, u} \cong \mathrm{C}_{q}\left[\widetilde{X}, \widetilde{X}^{\prime}\right], \quad \widetilde{\mathscr{X}_{\widetilde{X}}^{0}, u} \cong \mathrm{C}_{q}\left[Y, Y^{\prime}\right], \mathrm{C}_{q}=\mathrm{C}\left[q^{ \pm 1 / 4}\right] .
$$

See (4.3) and (4.11).

Theorem 4.7. For $u \neq 0$, let $\left\{\mathcal{V}_{n}: n \geq 0\right\}$ be the filtration of $\widetilde{\mathcal{H}}_{Y, \widetilde{X}^{-}}$ submodules in

$$
\widetilde{\mathscr{X}_{Y}^{1, u}} \quad \text { or } \quad \widetilde{\mathscr{X}_{\widetilde{X}}^{0, u}}
$$

defined in Proposition 4.6, (ii). For $n \geq 1$, we extend this filtration by adding the modules

$$
\mathcal{V}_{-n}=\mathrm{C}_{q}\left(\tilde{X}^{n}+\left(\tilde{X}^{\prime}\right)^{n}\right)+\mathcal{V}_{n+1} \text { or } \mathcal{V}_{-n}=\mathrm{C}_{q}\left(Y^{n}+\left(Y^{\prime}\right)^{n}\right)+\mathcal{V}_{n+1} \text {. }
$$

Then $\left\{\mathcal{V}_{n}: n \in \mathrm{Z}\right\}$ is a filtration of $\widetilde{\mathcal{H}}_{Y, \widetilde{X}}$-modules. The corresponding quotients of this filtration are the one-dimensional modules

$$
\begin{aligned}
& \widetilde{\mathrm{C}}_{Y}^{1, u}, \widetilde{\mathrm{C}}_{Y}^{0, q^{-1 / 2} u}, \widetilde{\mathrm{C}}_{Y}^{1, q^{-1 / 2} u}, \widetilde{\mathrm{C}}_{Y}^{0, q^{-1} u}, \ldots \text { for } \widetilde{\mathscr{X}}_{Y}^{1, u}, \\
& \widetilde{\mathrm{C}}_{\widetilde{X}}^{0, u}, \widetilde{\mathrm{C}}_{\widetilde{X}}^{1, q^{-1 / 2} u}, \widetilde{\mathrm{C}}_{\widetilde{X}}^{0, q^{-1 / 2} u}, \widetilde{\mathrm{C}}_{\widetilde{X}}^{1, q^{-1} u}, \ldots \text { for } \widetilde{\mathscr{X}}_{\widetilde{X}}^{0, u} .
\end{aligned}
$$

Proof. Let us consider only the case of (4.33). We can apply the intertwiner $\tilde{X}$ from part $(i i)$ of Lemma 4.5 to $e^{\prime}=e_{0}=1$; indeed, $T^{\prime}(1)=1$ and $Y^{\prime}(1)=u$. Automatically, $T(1)=0=Y(1)$. The element $e_{1}=\widetilde{e}^{\prime}=\widetilde{X}(1)$ will satisfy the conditions of part $($ ii $)$ in the Lemma and we can now apply $T^{\prime}$, which will result in

$$
e_{-1}=T^{\prime} \tilde{X}(1)=(T+1) \widetilde{X}(1)=\left(\widetilde{X}+\widetilde{X}^{\prime}\right)(1) .
$$

The next intertwiner in this chain will be $\widetilde{X}$ again, resulting in $e_{2}=$ $\widetilde{X}^{2}(1)$. Then $e_{-2}=\left(\widetilde{X}^{2}+\left(\widetilde{X}^{\prime}\right)^{2}\right)(1)$ and so on:

$$
e_{n}=\tilde{X}^{n}(1), \quad e_{-n}=\left(\widetilde{X}^{n}+\left(\tilde{X}^{\prime}\right)^{n}\right)(1) \text { for } n \geq 1 .
$$

They all will be $Y, Y^{\prime}$-eigenvectors. Since the elements $e_{-n}=\left(\widetilde{X}^{n}+\right.$ $\left.\left(\widetilde{X}^{\prime}\right)^{n}\right)(1)$ for $n>0$ satisfy $T\left(e_{-n}\right)=0$, the module $\widetilde{\mathcal{H}}_{Y, \widetilde{X}}\left(e_{-n}\right)$ divided by $\mathcal{V}_{n+1}$ is one-dimensional.

The consecutive quotients are exactly those claimed in the theorem; the description of the $Y, Y^{\prime}$-spectrum of $e_{ \pm n}$ readily follows from the lemma.

Actually, our construction is a counterpart of the chain of intertwiners in the bar-polynomial representation $\overline{\mathscr{X}}$ from $(2.35)$. Note that $u=1$ for the polynomial representation: $Y^{\prime}(1)=T^{\prime} \pi(1)=1$. Also $\widetilde{X}(1)=\tilde{\pi} T^{\prime}(1)=\Pi(1)$, i.e., the first intertwiner we used is actually $\Pi$ 
from (2.35). However, now the chain of intertwiners solves the problem of the decomposition of the bar-polynomial representation with respect to the action of $\widetilde{\mathcal{H}}_{Y, \tilde{X}}$, i.e., contains different information.

Using the dualization, one obtains the ascending filtrations in the remaining modules from (4.31).

Interestingly, using intertwiners in the nil-case can be done within the subalgebra $\widetilde{\mathcal{H}}_{Y, \tilde{X}}$, i.e., the classical (invertible) intertwiners $\widetilde{\pi}=\Pi$ and $\pi$ can be avoided; the latter is a counterpart of $\tilde{\pi}=\Pi$ for the modules induced from $\tilde{X}, \widetilde{X}^{\prime}$. It is directly related to Proposition 4.2, which can be now stated in its final form. We come to the following simple but fundamental theorem.

Theorem 4.8. Let us consider the elements $\left\{e_{n}: n \in \mathrm{Z}\right\}$ from (4.35) in the polynomial representation $\overline{\mathscr{X}}$. Then

$$
\bar{E}_{n}=q^{n^{2} / 4} e_{n}, n \in \mathrm{Z} .
$$

More explicitly,

$$
\begin{aligned}
\bar{E}_{n} & =q^{n^{2} / 4} \tilde{X}^{n}(1), n \geq 0, \\
\bar{E}_{-n} & =q^{n^{2} / 4}\left(\widetilde{X}^{n}+\left(\widetilde{X}^{\prime}\right)^{n}\right)(1), n>0 .
\end{aligned}
$$

Proof. The $e_{n}$ were constructed using the intertwiners $T^{\prime}$ and $\widetilde{X}$. Notice that $\widetilde{X}(1)=q^{-1 / 4} \Pi(1)$ and that

$$
\tilde{X} T^{\prime}=q^{-1 / 4} \Pi\left(T^{\prime}\right)^{2}=q^{-1 / 4} \Pi T^{\prime} .
$$

Hence we may just as well have used the intertwiners $T^{\prime}$ and $q^{-1 / 4} \Pi$, which are essentially those used to construct the $\bar{E}_{n}$. Comparing with (2.35), one obtains (4.36).

4.3. Shapovalov forms. Following [ChM], let us interpret the inner product $\langle f, g\rangle^{\prime}$ in the bar-polynomial representation, which was defined in (3.9), as a Shapovalov-type bilinear form.

4.3.1. Shapovalov trace. The Shapovalov trace on the core subalgebra $\widetilde{\mathcal{H}}_{Y, \widetilde{X}}$ is given by

$$
\left\langle\left(a\left(\tilde{X}^{\prime}\right)^{m^{\prime}+1}+b \tilde{X}^{m}\right)\left(T^{\prime}\right)^{\varsigma}\left(c Y^{n+1}+d\left(Y^{\prime}\right)^{n^{\prime}}\right)\right\rangle_{\psi}^{\prime} \stackrel{\text { def }}{=} b d
$$

where $\varsigma=0,1, m, m^{\prime}, n, n^{\prime} \geq 0$. The coefficients $a, b, c, d$ are from $\mathrm{C}_{q}=\mathrm{C}\left[q^{ \pm 1 / 4}\right]$. Recall that

$$
\tilde{X}=\psi\left(Y^{\prime}\right)=q^{1 / 4} Y X, \tilde{X}^{\prime}=\psi(Y)=q^{-1 / 4} X^{-1} Y^{\prime} .
$$


We use here the PBW part of Theorem 4.3.

Generally, the Shapovalov trace $\langle\cdot\rangle_{\alpha}$ requires a subalgebra $\mathcal{A}$ in $\widetilde{\mathcal{H}}_{Y, \widetilde{X}}$ or in $\overline{\mathcal{H}}$, its character $\chi: \mathcal{A} \rightarrow \mathrm{C}$ and an anti-involution $\alpha$. The defining relations are $\langle\alpha(A) H\rangle_{\alpha}=0=\langle H A\rangle_{\alpha}$ whenever $\chi(A)=0$ for any $H$.

Assuming that $\widetilde{\mathcal{H}}_{Y, \widetilde{X}}$ is linearly generated by the products from $\alpha(\mathcal{A}) \cdot \mathcal{A}$ (or $\overline{\mathcal{H}}$ is such) the dimension of the linear space of the Shapovalov traces (called DAHA-coinvariants in $[\mathrm{ChM}]$ ) is no greater than one. It is obvious, as is the relation $\left\langle H^{\alpha}\right\rangle_{\alpha}=\langle H\rangle_{\alpha}$ for $H \in \widetilde{\mathcal{H}}_{Y, \widetilde{X}}$.

Note that the definition from (4.40) is adjusted to the concrete induced module, which is the representation $\widetilde{\mathscr{X}}_{\widetilde{\pi}, Y}^{1,+}$ lifted to the barpolynomial representation of $\overline{\mathcal{H}}$. Namely, the algebra is $\mathcal{A}=\langle Y, T, \pi>$ and $\chi(H)=H(1)$ are as follows:

$$
Y(1)=T(1)=0, \pi(1)=1 \text { and } Y^{\prime}(1)=T^{\prime}(1)=1 .
$$

Generally, there are four possible choices for $\chi$ : one can combine $T(1)=$ $\epsilon=0,1$ with $\pi(1)=\delta= \pm 1$.

The functional $\langle\cdot\rangle_{\psi}^{\prime}$ naturally maps through the tilde-module

$$
\widetilde{\mathscr{X}_{Y, \widetilde{X}}^{1}+} \text {, which is naturally identified with } \overline{\mathscr{X}} \text {, }
$$

so it can be extended to $\overline{\mathcal{H H}}$. More explicitly, this extension is uniquely determined by the relations

$$
\begin{aligned}
& \left\langle A \pi^{\varsigma}\left(a Y^{n+1}+b\left(Y^{\prime}\right)^{n^{\prime}}\right)\right\rangle_{\psi}^{\prime}=b\langle A\rangle_{\psi}^{\prime} \\
& =\left\langle\left(a\left(\widetilde{X}^{\prime}\right)^{n+1}+b \widetilde{X}^{m^{\prime}}\right) \tilde{\pi}^{\varsigma} A\right\rangle_{\psi}^{\prime}
\end{aligned}
$$

for $A \in \overline{\mathcal{H}}$.

In particular, $\pi^{\varsigma}$ or $\tilde{\pi}^{\varsigma}$ can be inserted instead of $\left(T^{\prime}\right)^{\varsigma}$ in $(4.40)$; recall that $\tilde{\pi}=\psi(\pi)$. The formula $X=q^{1 / 4} \tilde{\pi} \pi$ readily results in $\langle X\rangle_{\psi}^{\prime}=q^{1 / 4}$. Also $T^{\prime} X^{-1}=X T$, which gives that $\left\langle X^{-1}\right\rangle_{\psi}^{\prime}=0$. Moreover,

$$
T^{\prime} X^{-n}=X^{n} T-X^{n-2} \text { for } n \geq 2 \text { and }\left\langle X^{-n}\right\rangle_{\psi}^{\prime}=-\left\langle X^{n-2}\right\rangle_{\psi}^{\prime} .
$$

Combining this relation with

$$
\left\langle X^{n}\right\rangle_{\psi}^{\prime}=\left\langle q^{n / 4}(\tilde{\pi} \pi)^{n}\right\rangle_{\psi}^{\prime}=\left\langle q^{n / 4}(\tilde{\pi} \pi)^{n-1}\right\rangle_{\psi}^{\prime}=\left\langle q^{n / 4} q^{(n-1) / 4} X^{1-n}\right\rangle_{\psi}^{\prime},
$$

we arrive at the formulas for the traces of arbitrary $X$-monomials:

$$
\left\langle X^{n}\right\rangle_{\psi}^{\prime}=-q^{n / 2-1 / 4}\left\langle X^{n-3}\right\rangle_{\psi}^{\prime} \text { for } n \geq 1,\left\langle X^{-n}\right\rangle_{\psi}^{\prime}=\left\langle X^{n-2}\right\rangle_{\psi}^{\prime},
$$

where $\langle 1\rangle_{\psi}^{\prime}=1,\langle X\rangle_{\psi}^{\prime}=q^{1 / 4},\left\langle X^{2}\right\rangle_{\psi}^{\prime}=0=\left\langle X^{-1}\right\rangle_{\psi}^{\prime},\left\langle X^{-2}\right\rangle_{\psi}^{\prime}=-1$. 
4.3.2. Shapovalov pairing. The next step is the pairing $\langle A, B\rangle_{\psi}^{\prime} \stackrel{\underline{\text { def }}}{=}$ $\left\langle A^{\psi} B\right\rangle_{\psi}^{\prime}$ for $A, B \in \overline{\mathcal{H}}$. It is symmetric and induces the anti-involution $\psi$ due to $\left\langle A^{\psi}\right\rangle_{\psi}^{\prime}=\langle A\rangle_{\psi}^{\prime}$. Its $q, t$-generalization is an important particular case of the theory of Shapovalov-type forms from [ChM].

Theorem 4.9. The pairing above naturally maps through $\overline{\mathscr{X}} \times \overline{\mathscr{X}}$, so we come to the following definition:

$$
\langle f, g\rangle_{\text {alg }}^{\prime} \stackrel{\text { def }}{=}\langle f(X), g(X)\rangle_{\psi}^{\prime}=\langle g, f\rangle_{\text {alg }}^{\prime} .
$$

The corresponding anti-involution is $\psi$ by construction. For any $q$ (including the roots of unity), the following holds:

$$
\langle f, g\rangle_{\text {alg }}^{\prime}=\langle f, g\rangle^{\prime} \text { for } f, g \in \overline{\mathscr{X}} \text {. }
$$

Proof. We use the irreducibility of $\overline{\mathscr{X}}$ for generic $q$ and the uniqueness of the bi-linear form in this representation associated with $\psi$ (equivalently, the PBW claim from Theorem 4.3).

Note that the Shapovalov construction automatically holds for arbitrary $q$. It is instructional to compare the coefficients of the (analytic) expansion of $\bar{\mu} \widetilde{\gamma}^{\prime}$ in terms of $X^{-n}$ from (2.43) with the entirely algebraic formulas for $\left\langle X^{n}\right\rangle_{\psi}^{\prime}$ from (4.42). The theorem states that they must coincide; they really do.

4.3.3. The tilde-case. We will use the identification of $\overline{\mathscr{X}}$ from Proposition 4.2:

$$
\widetilde{\mathscr{X}_{\widetilde{\pi}, Y}^{1,+}}=\widetilde{\mathscr{X}} \stackrel{\text { def }}{=}\left\{\sum_{l \geq 0} a_{l+1} \widetilde{X}^{l+1}(1)+a_{-l}\left(\widetilde{X}^{\prime}\right)^{l}(1)\right\}
$$

Here the coefficients are from $\mathrm{C}\left[q^{ \pm 1 / 4}\right]$. For the sake of definiteness, only the case of $\epsilon=1$ and $\delta=+$ will be considered here.

We now set

$$
\langle f, g\rangle_{\circ}=\left\langle f g \widetilde{\mu}_{\circ}\right\rangle,\langle f, g\rangle^{\prime}=\left\langle f g \widetilde{\mu \gamma^{\prime}}\right\rangle
$$

for $f, g \in \widetilde{\mathscr{X}}$, where the kernels of these pairings are those from (2.40) and (2.43) upon the substitution: $X \mapsto \tilde{X}, X^{-1} \mapsto \tilde{X}^{\prime}$. Recall that $\widetilde{X} \widetilde{X}^{\prime}=0=\widetilde{X}^{\prime} \widetilde{X}$. Explicitly,

$$
\begin{aligned}
& \widetilde{\mu}_{\circ}=\sum_{n=0}^{\infty}(-1)^{n} q^{\frac{n^{2}-n}{2}}\left(\widetilde{X}^{2 n}-\left(\widetilde{X}^{\prime}\right)^{2 n-2}\right), \\
& \widetilde{\mu \gamma}^{\prime}=\sum_{n=0}^{\infty} q^{n(n+2) / 12}\left(\widetilde{X}^{n+2}-\left(\widetilde{X}^{\prime}\right)^{n}\right), \text { where } n \neq 2 \bmod 3 .
\end{aligned}
$$


We claim that these inner products induce the anti-involutions $\diamond$ and $\psi$, respectively, for the action of $\overline{\mathcal{H}}$ in $\widetilde{\mathscr{X}_{Y, \widetilde{X}}^{1,+}}$. Recall that the anti-involution $\psi$ preserves the core subalgebra $\widetilde{\mathcal{H}}_{Y, \widetilde{X}}$ from (4.12).

The definitions of the Shapovalov trace and the pairing $\langle A, B\rangle_{\psi}^{\prime}$ in $\overline{\mathcal{H}}$ remain unchanged. The difference is that now we restrict this abstract pairing to "functions" of $\tilde{X}$.

Theorem 4.10. The Shapovalov-type form

$$
\langle f, g\rangle_{\text {alg }}^{\prime} \stackrel{\text { def }}{=}\langle\psi(f) g\rangle_{\psi}^{\prime}=\langle g, f\rangle_{\text {alg }}^{\prime} \text {, where } f \in \widetilde{\mathscr{X}} \ni g,
$$

is associated to the anti-involution $\psi$. For any $q$,

$$
\langle f, g\rangle_{\text {alg }}^{\prime}=\langle f, g\rangle^{\prime} \text { for } f, g \in \widetilde{\mathscr{X}} \text {. }
$$

4.3.4. Induction from tilde-X. Let us briefly discuss the changes needed for the modules induced from $\widetilde{X}$ :

$$
\widetilde{\mathscr{X}_{\pi, \widetilde{X}}^{0,-}}=\widetilde{\mathscr{Y}} \stackrel{\text { def }}{=}\left\{\sum_{l \geq 0} a_{l+1} Y^{l+1}(1)+a_{-l}\left(Y^{\prime}\right)^{l}(1)\right\} .
$$

See (4.3). We set

$$
\langle f(Y), g(Y)\rangle_{\circ}=\left\langle f(Y) g(Y) \widetilde{\mu}_{\circ}(Y)\right\rangle,\langle f(Y), g(Y)\rangle^{\prime}=\left\langle f g \widetilde{\mu \gamma}^{\prime}(Y)\right\rangle
$$

for $f(Y), g(Y) \in \widetilde{\mathscr{Y}}$, where the kernels of these pairings are from (2.40) and (2.43) upon the substitution: $X \mapsto Y^{\prime}, X^{-1} \mapsto Y$. Explicitly,

$$
\begin{aligned}
& \widetilde{\mu}_{\circ}(Y)=\sum_{n=0}^{\infty}(-1)^{n} q^{\frac{n^{2}-n}{2}}\left(\left(Y^{\prime}\right)^{2 n}-Y^{2 n-2}\right), \\
& \widetilde{\mu \gamma}^{\prime}(Y)=\sum_{n=0}^{\infty} q^{n(n+2) / 12}\left(\left(Y^{\prime}\right)^{n+2}-Y^{n}\right), \text { where } n \neq 2 \bmod 3 .
\end{aligned}
$$

We claim that these inner products induce the anti-involutions $\diamond$ and $\psi$ for the action of $\overline{\mathcal{H}}$ in $\widetilde{\mathscr{Y}}$ supplied with the action of $\overline{\mathcal{H}}$ via $\widetilde{\mathscr{X}}_{\pi, \widetilde{X}}^{1,+}$.

Concerning the Shapovalov-type construction, we need to reverse the order of operators in (4.40). The Shapovalov trace now reads:

$$
\left\langle\left(a\left(Y^{\prime}\right)^{m^{\prime}}+b Y^{m+1}\right)\left(T^{\prime}\right)^{\varsigma}\left(c \widetilde{X}^{n}+d\left(\tilde{X}^{\prime}\right)^{n^{\prime}+1}\right)\right\rangle_{\psi}^{\prime}=a c,
$$

where $\varsigma=0,1, m, m^{\prime}, n, n^{\prime} \geq 0$. Its extension to the whole $\overline{\mathcal{H}}$ is determined from

$$
\begin{aligned}
& \left\langle\left(a\left(Y^{\prime}\right)^{m^{\prime}}+b Y^{m+1}\right) \pi^{\varsigma} A\right\rangle_{\psi}^{\prime}=a\langle A\rangle_{\psi}^{\prime} \\
& =\left\langle A \widetilde{\pi}^{\varsigma}\left(a \tilde{X}^{n}+b\left(\tilde{X}^{\prime}\right)^{n^{\prime}+1}\right)\right\rangle_{\psi}^{\prime} .
\end{aligned}
$$


For instance, the relations from (4.50) are satisfied with $\pi^{\varsigma}$ or $\tilde{\pi}^{\varsigma}$ instead of $\left(T^{\prime}\right)^{\varsigma}(\varsigma=0,1)$.

Theorem 4.10 must be now stated in terms of the restriction of the pairing $\langle A, B\rangle_{\psi}^{\prime}$ to the "functions" of $Y$ :

$$
\langle f(Y), g(Y)\rangle_{\psi}^{\prime}=\langle f(Y), g(Y)\rangle^{\prime} \text { for } f(Y), g(Y) \in \widetilde{\mathscr{Y}}
$$

\section{SPINORS AND THE $q$-TODA THEORY}

In contrast to QMBP, the $q$-Toda operators (2.24) are nonsymmetric; the corresponding Dunkl operators were not expected to exist. The formulas from $[\mathrm{ChM}]$ were really surprising. They provided an exact counterpart of the $q, t$-representation $\mathcal{L}=Y+Y^{-1}$ (upon the restriction to the symmetric functions) for $\mathcal{L}$ from (1.26), but in the spinor sense. The introduction of the spinor-Dunkl operators made it possible to use DAHA methods at full potential, including the theory of the $q$-Whittaker functions. This construction can be readily extended to arbitrary root systems. The fact that the fundamental operators are of first order dramatically simplifies the theory. We will begin with the definition of the spinors following [ChM].

5.1. The spinors. Generally, $W$-spinors are needed in the DAHA theory. In the $A_{1}$-case, we will call them simply spinors. In this case, they really are connected with spinors from the theory of the Dirac operator (and with super-algebras). Under the rational degeneration, the Dunkl operator for $A_{1}$ becomes the square root of the (radial part of the) Laplace operator, i.e., the Dirac operator. However, this direct relation (and using super-variables) is a special feature of the root system $A_{1}$.

Let us mention that there are works in which DAHA is coupled with the Clifford algebra. This approach provides a formula for the Dirac operator in terms of Dunkl operators for any root systems; however, it is a different approach.

For practical calculations with spinors, the language of $\mathrm{Z}_{2}$-graded algebras can be used in the $A_{1}$-case. However, we prefer to do it in a way that does not rely on the special symmetry of the $A_{1}$-case and can be transferred to $W$-spinors for arbitrary root systems. 
5.1.1. The definitions. The spinors are simply pairs $\left\{f_{1}, f_{2}\right\}$ of elements (functions) from a space $\mathcal{F}$ with an action of $s$; the addition or multiplication (if applicable) of spinors is componentwise. The space of spinors will be denoted by $\widehat{\mathcal{F}}$.

The involution $s$ on spinors is defined as $s\left\{f_{1}, f_{2}\right\}=\left\{f_{2}, f_{1}\right\}$, so it does not involve the action of $s$ in $\mathcal{F}$. There is a "natural" embed$\operatorname{ding} \rho: \mathcal{F} \rightarrow \widehat{\mathcal{F}}$ mapping $f \mapsto f^{\rho}=\{f, s(f)\}$ and also the diagonal embedding $\delta: \mathcal{F} \rightarrow \widehat{\mathcal{F}}$ sending $f \mapsto f^{\delta}=\{f, f\}$. Accordingly, for an arbitrary operator $A$ acting in $\mathcal{F}, A^{\rho}=\{A, s(A)\}, A^{\delta}=\{A, A\}$. The images $f^{\rho}$ of $f \in \mathcal{F}$ are called functions (in contrast to spinors) or principal spinors (like for adeles).

For instance, for $\mathcal{F}=\mathscr{X}$,

$$
\begin{aligned}
& X^{\rho}:\left\{f_{1}, f_{2}\right\} \mapsto\left\{X f_{1}, X^{-1} f_{2}\right\}, \quad \Gamma^{\rho}:\left\{f_{1}, f_{2}\right\} \mapsto\left\{\Gamma\left(f_{1}\right), \Gamma^{-1}\left(f_{2}\right)\right\}, \\
& X^{\delta}:\left\{f_{1}, f_{2}\right\} \mapsto\left\{X f_{1}, X f_{2}\right\}, \quad \Gamma^{\delta}:\left\{f_{1}, f_{2}\right\} \mapsto\left\{\Gamma\left(f_{1}\right), \Gamma\left(f_{2}\right)\right\},
\end{aligned}
$$

where, recall, $\Gamma(X)=q^{1 / 2} X$. We simply put

$$
X^{\rho}=\left\{X, X^{-1}\right\}, \Gamma^{\rho}=\left\{\Gamma, \Gamma^{-1}\right\}, X^{\delta}=\{X, X\}, \Gamma^{\delta}=\{\Gamma, \Gamma\} .
$$

Obviously, $s^{\rho}=s=s^{\delta}$.

If a function $f \in \mathcal{F}$ or an operator $A$ acting in $\mathcal{F}$ has no super-index $\delta$, then they will be treated as $f^{\rho}, A^{\rho}$. I.e., by default, functions and operators are embedded into $\widehat{\mathcal{F}}$ and the algebra of spinor operators using $\rho$.

5.1.2. Spinor operators. If the operator $A$ is explicitly expressed as $\left\{A_{1}, A_{2}\right\}$, then $A_{1}$ and $A_{2}$ must be applied to the corresponding components of $f=\left\{f_{1}, f_{2}\right\}$. In the calculations below, $A_{i}$ may contain $s$. Then $A_{i}$ must be presented as $A_{i}^{\prime} \cdot s$, where $A_{i}^{\prime}$ contains no $s$; i.e., in practice $s$ must be placed on the right. In the operators in $\mathscr{X}$ we will consider, the commutation relations between $s$ and $X, \Gamma$ must be used when moving $s$. Then the component $i$ of $A f$ will be $A_{i}^{\prime}\left(f_{3-i}\right)$, i.e., $s$ placed on the right means the switch to the other component before applying $A_{i}^{\prime}$.

For instance, $\{\Gamma s, s-1\}\left(\left\{f_{1}, f_{2}\right\}\right)=\left\{\Gamma\left(f_{2}\right), f_{1}-f_{2}\right\}$.

We will frequently use the vertical mode for spinors:

$$
\left\{f_{1}, f_{2}\right\}=\left\{\begin{array}{c}
f_{1} \\
f_{2}
\end{array}\right\},\left\{A_{1}, A_{2}\right\}=\left\{\begin{array}{c}
A_{1} \\
A_{2}
\end{array}\right\} .
$$

5.1.3. The q-Toda operators via DAHA. The $q$-Toda spinor operator is the following symmetric (i.e., s-invariant) difference spinor operator

$$
\widehat{\mathcal{T}}=\left\{\Gamma^{-1}+\left(1-X^{-2}\right) \Gamma, \Gamma^{-1}+\left(1-X^{-2}\right) \Gamma\right\} .
$$


Its first component is the operator $R \mathbb{E}(\mathcal{L})$ from Section 2.2; we will use the notation and definitions from that section.

We claim that $\widehat{\mathcal{T}}$ can be represented as $\widehat{Y}+\widehat{Y}^{-1}$ upon the restriction to symmetric spinors, i.e., to $\{f, f\} \in \widehat{\mathcal{F}}$. The construction of the spinor-difference Dunkl operator $\widehat{Y}$ goes as follows.

Let us introduce the $\delta$-counterpart of $æ$ from (2.21):

$$
\begin{aligned}
& æ^{\delta}(\mathcal{A}) \stackrel{\text { def }}{=}\left(q^{k x} \Gamma_{k}^{-1}\right)^{\delta} \mathcal{A}\left(\Gamma_{k} q^{-k x}\right)^{\delta}, \\
& æ^{\delta}: X \mapsto \widetilde{t}^{-1 / 2} X, \Gamma \mapsto \widetilde{t}^{-1 / 2} \Gamma, s \mapsto s
\end{aligned}
$$

for the spinor constant $\widetilde{t}^{1 / 2} \stackrel{\text { def }}{=}\left\{t^{1 / 2}, t^{-1 / 2}\right\}$. Spinor constants are actually diagonal matrices, which may not commute with $s$ but commutes with $\Gamma$ and $X$. The spinor $R E$-construction is:

$$
R E^{\delta}: A \mapsto \lim _{t \rightarrow 0} æ^{\delta}(A) .
$$

It is of course very different from the procedure $R \mathbb{E}^{\rho}$ from Section 2.2. The spinor-Dunkl operators are $\widehat{Y}=R \mathbb{E}^{\delta}(Y), \widehat{Y}^{\prime}=R E^{\delta}\left(Y^{-1}\right)$. They are inverse to each other: $\widehat{Y} \widehat{Y}^{\prime}=1$. The following theorem is from [ChM].

Theorem 5.1. The map

$$
\begin{aligned}
& Y^{ \pm 1} \mapsto \widehat{Y}^{ \pm 1}, \breve{\pi} \mapsto R \mathbb{E}^{\delta}(X T), \\
& T \mapsto \widehat{T}=R \mathbb{E}^{\delta}\left(t^{1 / 2} T\right), T^{\prime} \mapsto \widehat{T}^{\prime}=R \mathbb{E}^{\delta}\left(t^{1 / 2} T^{-1}\right)
\end{aligned}
$$

can be extended to a representation of the algebra $\overline{\mathcal{H}}^{\varphi}$ in the space $\widehat{\mathscr{X}}$ of spinors over $\mathscr{X}=\mathrm{C}\left[q^{ \pm 1 / 4}\right]\left[X^{ \pm 1}\right]$. Correspondingly,

$$
\begin{aligned}
& X \mapsto R \mathbb{E}^{\delta}\left(t^{1 / 2} X\right)=R \mathbb{E}^{\delta}(X T) \circ \widehat{T}^{\prime}, \\
& X^{\prime} \mapsto R \mathbb{E}^{\delta}\left(t^{1 / 2} X^{-1}\right)=\widehat{T} \circ R \mathbb{E}^{\delta}(X T) .
\end{aligned}
$$

The commutativity of $T$ and $Y+Y^{-1}$ in $\overline{\mathcal{H}}^{\varphi}$ results in the s-invariance of $\widehat{Y}+\widehat{Y}^{-1}$ and the s-invariance of this operator upon its restriction to the space of $s$-invariant spinors; this operator is $\widehat{\mathcal{T}}$ from (5.1).

It is clear from the construction that all hat-operators preserve the space of spinors for the Laurent polynomials in terms of $X^{ \pm 1}$. Upon multiplication by the Gaussian, it contains the spinor polynomial representation, isomorphic to the Fourier image of the bar-polynomial representation times the Gaussian; see Section 2.2.3, formula (3.4) and 
Theorem 5.2 below. The reproducing kernel of the isomorphism between these two modules inducing $\sigma: \overline{\mathcal{H}} \rightarrow \overline{\mathcal{H}}^{\varphi}$ at the operator level is given by the spinor (nonsymmetric) $q$-Whittaker function.

\subsection{Spinor Whittaker function.}

5.2.1. Basic operators. Let us reproduce some formulas and calculations from [ChM], which will be needed to extend them to the case $|q|>1$ and, mainly, to establish the connection with the core-subalgebra. We will show below that the final formulas and certain steps in caclulating the limits are directly related to the core-induced modules.

We begin with $\widehat{Y}=\mathbb{E}^{\delta}(Y)=\lim _{t \rightarrow 0} \aleph^{\delta}(Y)$. Using formulas (5.3):

$$
\begin{aligned}
\aleph^{\delta}(Y) & =s \cdot\left(\widetilde{t}^{-1 / 2} \Gamma\right) \cdot\left(t^{1 / 2} s+\frac{t^{1 / 2}-t^{-1 / 2}}{\widetilde{t}^{-1} X^{2}-1} \cdot(s-1)\right) \\
& =t^{1 / 2} \widetilde{t}^{1 / 2} \Gamma^{-1}+\widetilde{t}^{1 / 2} \Gamma^{-1} \cdot \frac{t^{1 / 2}-t^{-1 / 2}}{\widetilde{t} X^{-2}-1} \cdot(1-s) \\
& =\left\{\begin{array}{c}
t \Gamma^{-1}+\Gamma^{-1} \frac{t-1}{t X^{-2}-1} \cdot(1-s) \\
\Gamma+\Gamma \frac{1-t^{-1}}{t^{-1} X^{2}-1} \cdot(1-s)
\end{array}\right\} \\
\stackrel{t \rightarrow 0}{\longrightarrow} \widehat{Y} & =\left\{\begin{array}{c}
\Gamma^{-1} \cdot(1-s) \\
\Gamma-\Gamma \cdot X^{-2} \cdot(1-s)
\end{array}\right\} .
\end{aligned}
$$

Recall that $\widetilde{t}^{1 / 2}=\left\{t^{1 / 2}, t^{-1 / 2}\right\}$. A little bit more involved calculation is needed for $\widehat{Y}^{\prime}=R E^{\delta}\left(Y^{-1}\right)$ :

$$
\begin{aligned}
x^{\delta}\left(Y^{-1}\right) & =\left(t^{-1 / 2} s+\frac{t^{-1 / 2}-t^{1 / 2}}{\widetilde{t} X^{-2}-1} \cdot(s-1)\right) \cdot\left(\widetilde{t}^{1 / 2} \Gamma^{-1} s\right) \\
& =\left(\frac{t^{-1 / 2} \widetilde{t} X^{-2}-t^{1 / 2}}{\widetilde{t} X^{-2}-1} \cdot s-\frac{t^{-1 / 2}-t^{1 / 2}}{\widetilde{t} X^{-2}-1}\right) \cdot\left(\widetilde{t}^{1 / 2} \Gamma^{-1} s\right) \\
& =\frac{t^{-1 / 2} \widetilde{t} X^{-2}-t^{1 / 2}}{\widetilde{t} X^{-2}-1} \widetilde{t}^{-1 / 2} \Gamma-\frac{t^{-1 / 2}-t^{1 / 2}}{\widetilde{t} X^{-2}-1} \widetilde{t}^{1 / 2} \Gamma^{-1} s \\
& =\left\{\begin{array}{c}
\frac{X^{-2}-1}{t X^{-2}-1} \Gamma-\frac{1-t}{t X^{-2}-1} \Gamma^{-1} s \\
\frac{t^{-1} X^{-t}}{t^{-1} X^{2}-1} \Gamma^{-1}-\frac{t^{-1}-1}{t^{-1} X^{2}-1} \Gamma s
\end{array}\right\} \stackrel{t \rightarrow 0}{\longrightarrow} \\
\widehat{Y}^{\prime} & =\left\{\begin{array}{c}
\left(1-X^{-2}\right) \Gamma+\Gamma^{-1} s \\
\Gamma^{-1}-\frac{1}{X^{2}} \Gamma s
\end{array}\right\} \\
& =\left\{\begin{array}{c}
1-X^{-2} \\
1
\end{array}\right\} \Gamma+\left\{\begin{array}{c}
1 \\
-X^{2}
\end{array}\right\} \Gamma^{-1} s .
\end{aligned}
$$


Automatically, $\widehat{Y} \widehat{Y}^{\prime}=1$. Now, as we claimed,

$$
\begin{aligned}
& \operatorname{RE}^{\delta}\left(Y+Y^{-1}\right)=\lim _{t \rightarrow 0} æ^{\delta}\left(Y+Y^{-1}\right) \\
= & \left\{\begin{array}{c}
\Gamma^{-1}(1-s)+\left(1-X^{-2}\right) \Gamma+\Gamma^{-1} s \\
\Gamma-\Gamma \frac{1}{X^{2}}(1-s)+\Gamma^{-1}-\frac{1}{X^{2}} \Gamma s
\end{array}\right\} \\
= & \left\{\begin{array}{c}
\Gamma^{-1}+\left(1-X^{-2}\right) \Gamma \\
\Gamma^{-1}+\left(1-X^{-2}\right) \Gamma
\end{array}\right\}(\bmod (\cdot)(s-1)) .
\end{aligned}
$$

For $X$ and $X^{-1}$, we have

$$
\begin{aligned}
& \text { (5.4) } \widehat{X}=R \mathbb{E}^{\delta}\left(t^{1 / 2} X\right)=\lim _{t \rightarrow 0} \aleph^{\delta}\left(t^{1 / 2} X\right)=\lim _{t \rightarrow 0} t^{1 / 2} \widetilde{t}^{-1 / 2} X=\left\{\begin{array}{c}
X \\
0
\end{array}\right\}, \\
& \widehat{X}^{\prime}=R \mathbb{E}^{\delta}\left(t^{1 / 2} X^{-1}\right)=\lim _{t \rightarrow 0} \aleph^{\delta}\left(t^{1 / 2} X^{-1}\right)=\lim _{t \rightarrow 0} t^{1 / 2} \widetilde{t}^{-1 / 2} X^{-1}=\left\{\begin{array}{c}
0 \\
X
\end{array}\right\} .
\end{aligned}
$$

Obviously, $\widehat{X} \widehat{X}^{\prime}=0$. Next,

$$
\begin{aligned}
& \widehat{T}=R \mathbb{E}^{\delta}\left(t^{1 / 2} T\right)=\lim _{t \rightarrow 0} æ^{\delta}\left(t^{1 / 2} T\right)=\left\{\begin{array}{c}
0 \\
s-1
\end{array}\right\}, \\
& \widehat{T}^{\prime}=R \mathbb{E}^{\delta}\left(t^{1 / 2} T^{-1}\right)=\lim _{t \rightarrow 0} æ^{\delta}\left(t^{1 / 2} T^{-1}\right)=\left\{\begin{array}{l}
1 \\
s
\end{array}\right\} .
\end{aligned}
$$

See $[\mathrm{ChM}]$ for more formulas and explicit verifications of the basic relations.

5.2.2. Using the components. Explicitly, the action of $\widehat{Y}$ and $\widehat{Y}^{\prime}$ on the spinors is as follows:

$$
\begin{aligned}
& \widehat{Y}\left(\left\{\begin{array}{l}
f_{1} \\
f_{2}
\end{array}\right\}\right)=\left\{\begin{array}{c}
\Gamma^{-1}\left(f_{1}-f_{2}\right) \\
\Gamma\left(f_{2}\right)-\Gamma\left(\frac{f_{2}-f_{1}}{X^{2}}\right)
\end{array}\right\}, \\
& \widehat{Y}^{\prime}\left(\left\{\begin{array}{l}
f_{1} \\
f_{2}
\end{array}\right\}\right)=\left\{\begin{array}{c}
\left(1-X^{-2}\right) \Gamma\left(f_{1}\right)+\Gamma^{-1}\left(f_{2}\right) \\
\Gamma^{-1}\left(f_{2}\right)-\frac{1}{X^{2}} \Gamma\left(f_{1}\right)
\end{array}\right\} .
\end{aligned}
$$

It is simple but not immediate to check the relation $\widehat{Y} \widehat{Y}^{\prime}=1$ and other identities for $\widehat{Y}^{ \pm 1}$ using the component formulas. The explicit formulas for $\widehat{T}$ and $\widehat{T}^{\prime}$ are:

$$
\widehat{T}\left(\left\{\begin{array}{l}
f_{1} \\
f_{2}
\end{array}\right\}\right)=\left\{\begin{array}{c}
0 \\
f_{1}-f_{2}
\end{array}\right\}, \quad \widehat{T}^{\prime}\left(\left\{\begin{array}{l}
f_{1} \\
f_{2}
\end{array}\right\}\right)=\left\{\begin{array}{l}
f_{1} \\
f_{1}
\end{array}\right\} .
$$


Finally,

$$
\begin{aligned}
\Re^{\delta}(X T) & =\left(\widetilde{t}^{-1 / 2} X\right)\left(t^{1 / 2} s+\frac{t^{1 / 2}-t^{-1 / 2}}{\widetilde{t}^{-1} X^{2}-1}(s-1)\right) \\
& =\widetilde{t}^{-1 / 2} t^{1 / 2} X s+\frac{X\left(\widetilde{t}^{-1 / 2} t^{1 / 2}-\widetilde{t}^{-1 / 2} t^{-1 / 2}\right)}{\widetilde{t}^{-1} X^{2}-1}(s-1) \\
& =\left\{\begin{array}{c}
X s \\
t X^{-1} s
\end{array}\right\}+\left\{\begin{array}{c}
\frac{X\left(1-t^{-1}\right)}{t^{-1} X^{2}-1}(s-1) \\
\frac{X^{-1}(t-1)}{t X^{-2}-1}(s-1)
\end{array}\right\} .
\end{aligned}
$$

Taking the limit $t \rightarrow 0$,

$$
\widehat{\pi}=\left\{\begin{array}{c}
X s \\
0
\end{array}\right\}+\left\{\begin{array}{c}
-X^{-1}(s-1) \\
X^{-1}(s-1)
\end{array}\right\}=\left\{\begin{array}{c}
X s-X^{-1}(s-1) \\
X^{-1}(s-1)
\end{array}\right\} .
$$

Using the components,

$$
\widehat{\pi}:\left\{\begin{array}{c}
f_{1} \\
f_{2}
\end{array}\right\} \mapsto\left\{\begin{array}{c}
X f_{2}+\frac{f_{1}-f_{2}}{X} \\
\frac{f_{1}-f_{2}}{X}
\end{array}\right\} .
$$

Check directly that $\widehat{\pi}^{2}=i d$.

This formula completes the "component presentation" of the hatmodule of $\overline{\mathcal{H}}^{\varphi}$ from Theorem 5.1:

$$
T, \breve{\pi}, Y \mapsto \widehat{T}, \widehat{\pi}, \widehat{Y}
$$

5.2.3. The main formula. Let us apply the procedure $R E^{\delta}$ to the global difference spherical function $G$ from (2.13). It was denoted by $\mathcal{E}_{q}(x, \lambda)$ in [Ch3], Section 5 (arbitrary reduced root systems). See [Sto1] for the $C^{\vee} C$-case. We need to use Proposition 2.33 to express the conjugated $E$-polynomials in terms of $E$-polynomials. Generally, this relation requires the action of $T_{w_{0}}$.

Following $[\mathrm{ChM}]$, we arrive at the spinor (nonsymmetric) generalization of $\mathcal{W}$ from $(2.27)$ :

$$
\begin{array}{r}
\Omega(X, \Lambda)=\left(\widetilde{\gamma}^{\prime}(X) \widetilde{\gamma}^{\prime}(\Lambda)\right)^{-1}\left(1+\sum_{m=1}^{\infty} q^{m^{2} / 4}\left(\frac{\bar{E}_{-m}(\Lambda)}{\prod_{s=1}^{m}\left(1-q^{s}\right)}\left\{\begin{array}{c}
X^{m} \\
q^{m} X^{m}
\end{array}\right\}\right.\right. \\
\left.\left.+\frac{\bar{E}_{m}(\Lambda)}{\prod_{s=1}^{m-1}\left(1-q^{s}\right)}\left\{\begin{array}{c}
0 \\
X^{m}
\end{array}\right\}\right)\right) .
\end{array}
$$

Using the Pieri rules from (2.37), it can be presented as follows:

$$
\Omega=\left(\widetilde{\gamma}^{\prime}(X) \widetilde{\gamma}^{\prime}(\Lambda)\right)^{-1} \sum_{m=0}^{\infty} \frac{q^{m^{2} / 4}}{\prod_{s=1}^{m}\left(1-q^{s}\right)}\left\{\begin{array}{c}
X^{m} \bar{E}_{-m}(\Lambda) \\
X^{m} \Lambda^{-1} \bar{E}_{m+1}(\Lambda)
\end{array}\right\} .
$$

Either of these two presentations readily gives that the spinor symmetrization of $\Omega$ is $\{\mathcal{W}, \mathcal{W}\}$ for the symmetric $q$-Whittaker function 
$\mathcal{W}$. We need to apply the symmetrizer $T^{\prime}=T+1$ to $\Omega$, equivalently, make the second component equal to the first one; see (5.5). Note that $\Lambda$ is a non-spinor variable.

The spinor function $\Omega$ intertwines the bar-representation of $\overline{\mathcal{H}}$ and the hat-representation of $\overline{\mathcal{H}}^{\varphi}$. Namely,

$$
\begin{aligned}
& \widehat{Y}(\Omega)=\Lambda^{-1}(\Omega), \quad \widehat{X}(\Omega)=\bar{Y}_{\Lambda}^{\prime}(\Omega), \quad \widehat{X}^{\prime}(\Omega)=\bar{Y}_{\Lambda}(\Omega), \\
& \widehat{\pi}(\Omega)=\pi_{\Lambda}(\Omega), \quad \widehat{T}(\Omega)=\bar{T}_{\Lambda}(\Omega), \quad \widehat{T}^{\prime}(\Omega)=\bar{T}_{\Lambda}^{\prime}(\Omega),
\end{aligned}
$$

where $\bar{Y}_{\Lambda}^{\prime}, \bar{Y}_{\Lambda}, \pi_{\Lambda}, \bar{T}_{\Lambda}$ act on the argument $\Lambda$; the other operators are $X$-operators. Recall that $\widehat{\pi}$ is the action of $\breve{\pi}=X T$ in the spinor representation.

These (and other related identities) follow from the general theory for arbitrary reduced root systems (at least, in the twisted case). However, in the rank one case (and for $A_{n}$ ), one can use the Pieri rules from (2.36),(2.37) and formulas (2.38), (2.39) for the direct verification. See [ChM] for explicit calculations. For instance,

$$
\widetilde{\gamma}^{\prime}(X) \widehat{Y} \widetilde{\gamma}^{\prime}(X)^{-1}\left\{\begin{array}{c}
f_{1} \\
f_{2}
\end{array}\right\}=q^{\frac{1}{4}}\left\{\begin{array}{c}
X^{-1} \Gamma^{-1}\left(f_{1}-f_{2}\right) \\
X \Gamma\left(f_{2}\right)+q^{-1} \frac{\Gamma\left(f_{1}-f_{2}\right)}{X}
\end{array}\right\} .
$$

Formulas (5.11), (5.6) and (5.5) were used in [ChM] to introduce the spinor-polynomial representation.

Theorem 5.2. The space

$$
\mathscr{X}_{\text {spin }} \stackrel{\text { def }}{=} \mathrm{C} \oplus\left(\oplus_{m=1}^{\infty}\left(\mathrm{C}\left\{X^{m}, 0\right\} \oplus \mathrm{C}\left\{0, X^{m}\right\}\right)\right) .
$$

is an irreducible $\overline{\mathcal{H}}^{\varphi}$-submodule of the space of spinors over $\mathrm{C}\left[X^{ \pm 1}\right]$ supplied with the twisted action:

$$
\overline{\mathcal{H}}^{\varphi} \ni A \mapsto q^{-x^{2}} \widehat{A} q^{x^{2}}
$$

Equivalently, $\mathscr{X}_{\text {spin }}$ is invariant and irreducible under the action of operators $\widehat{T}, \widehat{\pi}$ and $q^{-x^{2}} \widehat{Y} q^{x^{2}}$.

\subsection{Algebraic theory.}

5.3.1. Relation to tilde-modules. It is important that the $\overline{\mathcal{H}}^{\varphi}$-module $\mathscr{X}_{\text {spin }}$ can be identified with the induced $\overline{\mathcal{H}}$-module $\widetilde{\mathscr{X}}_{\pi, \widetilde{X}}^{0,-}$ (where $\epsilon=0$ and $\delta=-1)$. The identification goes as follows. 
Theorem 5.3. Let us define the $\mathrm{C}-$ linear map $\chi: \widetilde{\mathscr{X}}_{\pi, \widetilde{X}}^{0,-} \rightarrow \mathscr{X}_{\text {spin }}$ by

$$
1 \mapsto\{1,1\}, Y^{m} \mapsto\left\{X^{m}, 0\right\},\left(Y^{\prime}\right)^{m} \mapsto\left\{0, X^{m}\right\}, q \mapsto q^{-1} .
$$

for $m>0$. It induces the following isomorphism on operators:

$$
\begin{aligned}
& \chi: \overline{\mathcal{H}} \rightarrow \overline{\mathcal{H}}^{\varphi}, T \mapsto-T^{\prime}, \pi \mapsto-\breve{\pi}, X^{ \pm 1} \mapsto Y^{ \pm 1}, q \mapsto q^{-1}, \\
& \chi\left(T^{\prime}\right)=1-T^{\prime}=-T, \chi(Y)=\breve{\pi} T^{\prime}=X, \chi\left(Y^{\prime}\right)=T \breve{\pi}=X^{\prime} .
\end{aligned}
$$

Proof. Formula (5.11) gives that $q^{-x^{2}} \widehat{Y} q^{x^{2}}(\{1,1\})=q^{1 / 4}\{0, X\}$,

$$
\begin{aligned}
& q^{-x^{2}} \widehat{Y} q^{x^{2}}\left\{\begin{array}{c}
X^{m} \\
0
\end{array}\right\}=q^{\frac{1}{4}}\left\{\begin{array}{c}
X^{-1} q^{-\frac{m}{2}} X^{m} \\
q^{-1} X^{-1} q^{\frac{m}{2}} X^{m}
\end{array}\right\} \\
& =q^{-\frac{1}{4}}\left(q^{-\frac{m-1}{2}}\left\{\begin{array}{c}
X^{m-1} \\
0
\end{array}\right\}+q^{\frac{m-1}{2}}\left\{\begin{array}{c}
0 \\
X^{m-1}
\end{array}\right\}\right), \\
& q^{-x^{2}} \widehat{Y} q^{x^{2}}\left\{\begin{array}{c}
0 \\
X^{m}
\end{array}\right\}=q^{\frac{1}{4}}\left\{\begin{array}{c}
-X^{-1} q^{-\frac{m}{2}} X^{m} \\
X q^{\frac{m}{2}} X^{m}-q^{-1} X^{-1} q^{\frac{m}{2}} X^{m}
\end{array}\right\} \\
& =q^{-\frac{1}{4}}\left(q^{\frac{m+1}{2}}\left\{\begin{array}{c}
0 \\
X^{m+1}
\end{array}\right\}-q^{-\frac{m-1}{2}}\left\{\begin{array}{c}
X^{m-1} \\
0
\end{array}\right\}\right. \\
& \left.-q^{\frac{m-1}{2}}\left\{\begin{array}{c}
0 \\
X^{m-1}
\end{array}\right\}\right) \text {, where } m \geq 1 .
\end{aligned}
$$

Compare with (4.9); the action of $Y \in \overline{\mathcal{H}}^{\varphi}$ coincides with that of $X \in \overline{\mathcal{H}}$ upon the identification given by (5.12).

Next, the operator $\widehat{T}^{\prime}=\{1, s\}$ coincides with $-T$ defined by (4.6):

$$
\begin{aligned}
& -T\left(\sum_{l \geq 0} a_{l+1} Y^{l+1}+a_{-l}\left(Y^{\prime}\right)^{l}\right)(1) \\
& =\sum_{l \geq 0} a_{l+1}\left(Y^{l+1}+\left(Y^{\prime}\right)^{l+1}\right)(1) .
\end{aligned}
$$

Finally, the component presentation of $\widehat{\pi}$ from (5.6) results in

$$
\begin{aligned}
& \widehat{\pi}\left(\left\{\begin{array}{c}
1 \\
1
\end{array}\right\}\right)=\left\{\begin{array}{c}
X \\
0
\end{array}\right\}, \widehat{\pi}\left(\left\{\begin{array}{c}
X^{m} \\
0
\end{array}\right\}\right)=\left\{\begin{array}{l}
X^{m-1} \\
X^{m-1}
\end{array}\right\}, \\
& \widehat{\pi}\left(\left\{\begin{array}{c}
0 \\
X^{m}
\end{array}\right\}\right)=\left\{\begin{array}{c}
X^{m+1} \\
0
\end{array}\right\}-\left\{\begin{array}{l}
X^{m-1} \\
X^{m-1}
\end{array}\right\} .
\end{aligned}
$$

This coincides with $-\pi \in \overline{\mathcal{H}}$ defined by (4.8).

We see that $\chi$ is essentially an involution and can be used equally well to go from $\overline{\mathcal{H}}^{\varphi}$ to $\overline{\mathcal{H}}$. However we prefer to use $\chi^{-1}$ for the inverse map. Note that $\chi$ is nothing but

$$
\nu_{-} \varepsilon: \overline{\mathcal{H}} \rightarrow \overline{\mathcal{H}}^{\varphi \dagger} \rightarrow \overline{\mathcal{H}}^{\varphi},
$$


where $\nu_{ \pm}$are defined in (3.15), $\varepsilon$ is the bar-restriction of that from (1.4), naturally sending

$$
\varepsilon: \overline{\mathcal{H}} \rightarrow \overline{\mathcal{H}}^{\varphi \dagger}: T \mapsto\left(T^{\dagger}\right)^{\prime}, X \mapsto Y, Y \mapsto X, q \mapsto q^{-1} .
$$

5.3.2. Algebraic Whittaker function. Let us apply the identification $\chi^{-1}$ from (5.12) to $\Omega(X, \Lambda)$. We obtain

$$
\begin{aligned}
\Omega_{\mathrm{alg}}\left(Y, Y^{\prime} ; \Lambda\right) \stackrel{\text { def }}{=} & q^{-\lambda^{2}}\left(1+\sum_{m=1}^{\infty} q^{-\frac{m^{2}}{4}}\left(\frac{\left(\bar{E}_{m}(\Lambda)\right)^{*}}{\prod_{s=1}^{m-1}\left(1-q^{-s}\right)}\left(Y^{\prime}\right)^{m}\right.\right. \\
& \left.\left.+\frac{\left(\bar{E}_{-m}(\Lambda)\right)^{*}}{\prod_{s=1}^{m}\left(1-q^{-s}\right)}\left(Y^{m}+q^{-m}\left(Y^{\prime}\right)^{m}\right)\right)\right) .
\end{aligned}
$$

Here it is convenient to use the Gaussian $q^{-\lambda^{2}}$ instead of $\widetilde{\gamma}^{\prime}(\Lambda)$; it is understood as a formal symbol satisfying the standard relations. We drop the Gaussian for the spinor variables because it is already incorporated into the representation and the conjugation by this function is included in $\chi$.

The coefficients of the summation in (5.18) belong to $\overline{\mathscr{X}}_{\Lambda}^{\dagger} \otimes \widetilde{\mathscr{X}}_{\pi, \widetilde{X}}^{0,-}$. Note $\dagger$, which appears due to the conjugation of $\bar{E}_{m}(\Lambda) ; \overline{\mathscr{X}}_{\Lambda}^{\dagger}$ is a module over $\overline{\mathcal{H}}^{\dagger}$ (not over $\overline{\mathcal{H}}$ ).

Applying $\chi^{-1}$ to (5.9), the relations satisfied by $\Omega_{\text {alg }}$ are

$$
\chi^{-1}\left(H^{\varphi}\right)\left(\Omega_{\text {alg }}\right)=\eta\left(H_{\Lambda}\right)\left(\Omega_{\text {alg }}\right), \text { where } H \in \overline{\mathcal{H}} .
$$

Here $\varphi$ is from (3.3) and $\eta$ is from (3.19). Using that

$$
\chi^{-1}=\varepsilon \nu_{-}=\nu_{-} \varepsilon \text { and } \nu_{-} \varepsilon \varphi \eta=\nu_{-} * \eta=\nu_{-} \diamond,
$$

we can rewrite (5.19) as follows:

$$
\left(H^{\diamond}\right)\left(\Omega_{\mathrm{alg}}\right)=\nu_{-}\left(H_{\Lambda}\right)\left(\Omega_{\mathrm{alg}}\right), \quad \text { where } H \in \overline{\mathcal{H}} .
$$

Explicitly,

$$
\begin{aligned}
X\left(\Omega_{\mathrm{alg}}\right) & =\Lambda\left(\Omega_{\mathrm{alg}}\right), \quad-\pi\left(\Omega_{\mathrm{alg}}\right)=\pi_{\Lambda}\left(\Omega_{\mathrm{alg}}\right), \\
-T\left(\Omega_{\mathrm{alg}}\right) & =\bar{T}_{\Lambda}^{\dagger}\left(\Omega_{\mathrm{alg}}\right), \quad-T^{\prime}\left(\Omega_{\mathrm{alg}}\right)=\left(\bar{T}_{\Lambda}^{\dagger}-1\right)\left(\Omega_{\mathrm{alg}}\right), \\
Y\left(\Omega_{\mathrm{alg}}\right) & =\bar{T}_{\Lambda}^{\dagger} \pi\left(\Omega_{\mathrm{alg}}\right), \quad Y^{\prime}\left(\Omega_{\mathrm{alg}}\right)=\pi\left(\bar{T}_{\Lambda}^{\dagger}-1\right)\left(\Omega_{\mathrm{alg}}\right) .
\end{aligned}
$$

Theorem 5.4. (i) Let us define the transform $\mathbf{B}: \overline{\mathscr{X}}_{\Lambda} q^{-\lambda^{2}} \rightarrow \widetilde{\mathscr{X}}_{\pi, \widetilde{X}}^{0,-}$ by

$$
\mathbf{B}\left(f(\Lambda) q^{-\lambda^{2}}\right)\left(Y, Y^{\prime}\right) \stackrel{\text { def }}{=}\left(\left(\Omega_{\mathrm{alg}}\left(Y, Y^{\prime} ; \Lambda\right), f(\Lambda) q^{-\lambda^{2}}\right)\right) \text { 。 }
$$


for $((),) \circ$ from (3.22). Then it induces $\nu_{-} \eta: \overline{\mathcal{H}} \rightarrow \overline{\mathcal{H}}^{\varphi}$ on operators:

$$
\mathbf{B}\left(H\left(f(\Lambda) q^{-\lambda^{2}}\right)\right)=\left(\nu_{-} \eta(H)\right)\left(\mathbf{B}\left(f(\Lambda) q^{-\lambda^{2}}\right)\right) .
$$

(ii) Using $M_{m}^{Y}=Y^{m}+\left(Y^{\prime}\right)^{m}$ for $m>0$ and $M_{0}^{Y}=1$,

$$
\begin{aligned}
& \mathbf{B}\left(\bar{E}_{-m}(\Lambda) q^{-\lambda^{2}}\right)=q^{-\frac{m^{2}}{4}} M_{m}^{Y}(1) \text { for } m \geq 0, \\
& \mathbf{B}\left(\bar{E}_{+m}(\Lambda) q^{-\lambda^{2}}\right)=q^{-\frac{m^{2}}{4}} Y^{m}(1) \text { for } m \geq 1 .
\end{aligned}
$$

We use here $H(1)$ in $\widetilde{\mathscr{X}_{\pi, \widetilde{X}}^{0,-}}$; see the identification from (4.3).

5.3.3. Justification. Theorem 5.4 is essentially a variant of Theorem 2.1, (ii) adjusted to the nil-setting. It induces $\nu_{-} \eta$ on operators by construction. However, part $(i i)$, concerning the explicit formulas, is not quite obvious. The following transformation of the expression in (5.18) is needed.

Lemma 5.5. In terms of the polynomials $\left\{\bar{E}_{m}^{\dagger}\right\}$ from (3.21),

$$
\begin{aligned}
\Omega_{\mathrm{alg}}\left(Y, Y^{\prime} ; \Lambda\right)=q^{-\lambda^{2}} & \left(\sum_{m=1}^{\infty} q^{-\frac{(m-1)^{2}}{4}} M_{m-1}^{Y}(1) \frac{\bar{E}_{1-m}^{\dagger}(\Lambda)}{\prod_{s=1}^{m-1}\left(1-q^{-s}\right)}\right. \\
& \left.+\sum_{m=0}^{\infty} q^{-\frac{(1+m)^{2}}{4}} Y^{m+1}(1) \frac{\bar{E}_{1+m}^{\dagger}(\Lambda)}{\prod_{s=1}^{m}\left(1-q^{-s}\right)}\right) .
\end{aligned}
$$

Proof. To avoid misunderstanding, let us calculate a couple of first terms in the right-hand side. The term with $m=1$ in the first summation is 1 . The term with $m=0$ in the second summation is $q^{-1 / 4} Y(1) E_{1}^{\dagger}(\Lambda)=q^{-1 / 4} \Lambda Y$, since $\bar{E}_{1}^{\dagger}(\Lambda)=\Lambda$. There is another appearance of $Y$ due to $M_{1}^{Y}$; the corresponding term is

$$
q^{-\frac{1}{4}} Y(1) \frac{\bar{E}_{-1}^{\dagger}(\Lambda)}{1-q^{-1}}, \text { where } \bar{E}_{-1}^{\dagger}(\Lambda)=\Lambda^{-1}+q^{-1} \Lambda \text {. }
$$

Thus the total coefficient of $Y$ is

$$
q^{-1 / 4}\left(\left(1+\frac{q^{-1}}{1-q^{-1}}\right) \Lambda+\frac{1}{1-q^{-1}} \Lambda^{-1}\right)=q^{-1 / 4} \frac{\Lambda+\Lambda^{-1}}{1-q^{-1}} .
$$

This is exactly the coefficient of $Y$ from the original formula (5.18), which is $q^{-1 / 4} \bar{E}_{-1}(\Lambda)^{*} /\left(1-q^{-1}\right)$.

The general verification is based on the formulas

$$
\bar{E}_{m}(\Lambda)^{*}=\Lambda^{-1} \bar{E}_{1-m}^{\dagger}, X\left(\Omega_{\mathrm{alg}}\right)=\Lambda\left(\Omega_{\mathrm{alg}}\right) .
$$


One has:

$$
\begin{aligned}
& \Omega_{\mathrm{alg}}\left(Y, Y^{\prime} ; \Lambda\right)=\Lambda X^{-1}\left(\Omega_{\mathrm{alg}}\left(Y, Y^{\prime} ; \Lambda\right)\right)= \\
& q^{-\lambda^{2}}\left(\Lambda X^{-1}(1)+\sum_{m=1}^{\infty} q^{-\frac{m^{2}}{4}}\left(\frac{\bar{E}_{1-m}^{\dagger}(\Lambda)}{\prod_{s=1}^{m-1}\left(1-q^{-s}\right)} X^{-1}\left(\left(Y^{\prime}\right)^{m}\right)\right.\right. \\
& \left.\left.+\frac{\bar{E}_{1+m}^{\dagger}(\Lambda)}{\prod_{s=1}^{m}\left(1-q^{-s}\right)} X^{-1}\left(Y^{m}+q^{-m}\left(Y^{\prime}\right)^{m}\right)\right)(1)\right) .
\end{aligned}
$$

Now we can use the formulas (4.9) for $\delta=-1$ :

$$
\begin{gathered}
X^{-1}\left(Y^{\prime}\right)=(-\delta) q^{\frac{1}{4}}, X^{-1}(1)=(-\delta) q^{-\frac{1}{4}} Y(1) \\
X^{-1}\left(\left(Y^{\prime}\right)^{m}\right)=(-\delta) q^{-\frac{1}{4}} q^{+m / 2}\left(Y^{m-1}+\left(Y^{\prime}\right)^{m-1}\right)(1), \\
X^{-1}\left(Y^{m}+q^{-m}\left(Y^{\prime}\right)^{m}\right)=(-\delta) q^{-\frac{1}{4}} q^{-m / 2} Y^{m+1}(1)(m \geq 2) .
\end{gathered}
$$

It concludes the verification of the lemma.

According to the general approach from (2.11), the series from (5.25) is exactly the reproducing kernel for $\mathbf{B}$ from the theorem for the basis $\left\{f_{n}=\bar{E}_{n}(\Lambda) q^{-\lambda^{2}}\right\}$ and basis $\left\{f_{n}^{\prime}=\bar{E}_{n}^{\dagger}(\Lambda) q^{-\lambda^{2}}\right\}$ orthogonal to each other with respect to $(()$,$) o. Thus,$

$$
\begin{aligned}
& \Omega_{\mathrm{alg}}\left(Y, Y^{\prime} ; \Lambda\right)=\sum_{m \in \mathrm{z}} \frac{\bar{E}_{m}^{\dagger}(\Lambda) q^{-\lambda^{2}} \cdot \mathbf{B}\left(\bar{E}_{m}(\Lambda) q^{-\lambda^{2}}\right)}{\left(\left(\bar{E}_{m}^{\dagger}(\Lambda) q^{-\lambda^{2}}, \bar{E}_{m}(\Lambda) q^{-\lambda^{2}}\right)\right)_{\circ}}, \\
& \left(\left(\Omega_{\mathrm{alg}}\left(Y, Y^{\prime} ; \Lambda\right), q^{-\lambda^{2}} \bar{E}_{m}(\Lambda)\right)\right)=\mathbf{B}\left(q^{-\lambda^{2}} \bar{E}_{m}\right) .
\end{aligned}
$$

See (3.28) for the values of $\left(\left(\bar{E}_{m}^{\dagger} q^{-\lambda^{2}}, \bar{E}_{m} q^{-\lambda^{2}}\right)\right)_{\circ}=\left(\left(\bar{E}_{m}^{\dagger}, \bar{E}_{m}(\Lambda)\right)\right)_{\circ}$.

It is instructional to check directly the following corollary of the theorem. Since, $\tau_{+}^{-1}(Y)_{\Lambda}=q^{1 / 4} X^{-1} Y$ and $\tau_{+}^{-1}\left(Y^{\prime}\right)_{\Lambda}=q^{-1 / 4} Y^{\prime} X$ diagonalize $\bar{E}_{n}(\Lambda) q^{-\lambda^{2}}$ the operators

$$
\begin{aligned}
& \mathcal{Y}=\eta \tau_{+}^{-1}(Y)=q^{-1 / 4} X \pi T^{-1}=q^{1 / 4} \pi T X=q^{1 / 4} Y X \\
& \mathcal{Y}^{\prime}=\eta \tau_{+}^{-1}\left(Y^{\prime}\right)=q^{-1 / 4} X^{-1} Y^{\prime}
\end{aligned}
$$

must diagonalize the images of $\bar{E}_{n}(\Lambda) q^{-\lambda^{2}}$ under B. Here $\nu_{-}$acts trivially on $X, Y$. 
More exactly, the eigenvalues must be preserved too. Due to (2.38) and (2.39):

$$
\begin{aligned}
& \bar{Y}\left(\bar{E}_{n}\right)=\left\{\begin{array}{cc}
q^{-|n| / 2} \bar{E}_{n}, & n>0, \\
0, & n \leq 0 .
\end{array}\right\}, \\
& \bar{Y}^{\prime}\left(\bar{E}_{n}\right)=\left\{\begin{array}{cc}
q^{-|n| / 2} \bar{E}_{n}, & n \leq 0, \\
0, & n>0 .
\end{array}\right\} .
\end{aligned}
$$

So we must have:

$$
\begin{aligned}
& \mathcal{Y}\left(Y^{n}\right)=q^{-n / 2} Y^{n}(n>0), \quad \mathcal{Y}\left(M_{n}^{Y}\right)=0(n \geq 0), \\
& \mathcal{Y}^{\prime}\left(M_{n}^{Y}\right)=q^{-n / 2} Y^{n}(n \geq 0), \mathcal{Y}^{\prime}\left(Y^{n}\right)=0(n>0) .
\end{aligned}
$$

This readily follows from (5.28) for $\mathcal{Y}^{\prime}$. For $\mathcal{Y}$, use these relations multiplied by $X$ :

$$
\begin{aligned}
& X(1)=(-\delta) q^{-\frac{1}{4}} Y^{\prime}, X(Y)=(-\delta) q^{\frac{1}{4}} \\
& X\left(Y^{m-1}+\left(Y^{\prime}\right)^{m-1}\right)=(-\delta) q^{\frac{1}{4}} q^{-m / 2}\left(Y^{\prime}\right)^{m}, \\
& X\left(Y^{m+1}\right)=(-\delta) q^{\frac{1}{4}} q^{m / 2}\left(Y^{m}+q^{-m}\left(Y^{\prime}\right)^{m}\right)(m \geq 2) .
\end{aligned}
$$

More conceptually, follow Lemma 4.5.

5.3.4. Omitting the conjugation. According to the general formula from (2.12), if we use the pairing $\langle f, g\rangle_{\circ}$ from (3.11) corresponding to the anti-involution $\diamond$, the corresponding transform will represent $\nu_{-}$, i.e., it will essentially be an isomorphism of $\overline{\mathcal{H}}$ modules. Let us demonstrate this now. We will use directly the presentation of $\Omega_{\text {alg }}$ from (5.18); there is no need for $(5.25)$.

Now $\left\{f_{n}=\bar{E}_{n}^{\dagger}(\Lambda) q^{\lambda^{2}}\right\}$ and $\left\{f_{n}^{\prime}=\left(\bar{E}_{n}(\Lambda) q^{\lambda^{2}}\right)^{*}\right\}$ are orthogonal to each other with respect to $\langle,\rangle_{\circ}$. We come to the following counterpart of Theorem 5.4.

Theorem 5.6. (i) Let us define $\mathbf{F}: \overline{\mathscr{X}}_{\Lambda}^{\dagger} q^{+\lambda^{2}} \rightarrow \widetilde{\mathscr{X}_{\pi, \widetilde{X}}^{0,-}}$ by

$$
\mathbf{F}\left(f(\Lambda) q^{+\lambda^{2}}\right)\left(Y, Y^{\prime}\right) \stackrel{\text { def }}{=}\left\langle\Omega_{\mathrm{alg}}\left(Y, Y^{\prime} ; \Lambda\right), f(\Lambda) q^{-\lambda^{2}}\right\rangle_{\circ}
$$

for $\langle,\rangle_{\circ}$ from (3.11). Then

$$
\begin{aligned}
& \mathbf{F}\left(H\left(f(\Lambda) q^{+\lambda^{2}}\right)\right)=\nu_{-}(H)\left(\mathbf{F}\left(f(\Lambda) q^{+\lambda^{2}}\right)\right), \\
& \mathbf{F}\left(\bar{E}_{-m}^{\dagger}(\Lambda) q^{+\lambda^{2}}\right)=q^{-\frac{m^{2}}{4}}\left(Y^{m}+q^{-m}\left(Y^{\prime}\right)^{m}\right)(1) \text { for } m \geq 0, \\
& \mathbf{F}\left(\bar{E}_{+m}^{\dagger}(\Lambda) q^{+\lambda^{2}}\right)=q^{-\frac{m^{2}}{4}}\left(Y^{\prime}\right)^{m}(1) \text { for } m \geq 1 .
\end{aligned}
$$


Similar to the explicit calculation above, let us check that the eigenfunctions really go to the corresponding eigenfunctions under $\mathbf{F}$. The polynomials $\bar{E}_{n}^{\dagger}(\Lambda)=\Lambda\left(\bar{E}_{1-n}\right)^{*}$ solve the following eigenvalue problem:

$$
\begin{aligned}
& \left(\bar{Y}^{\dagger}\right)^{\prime}\left(\bar{E}_{n}^{\dagger}\right)=\left\{\begin{array}{cc}
q^{|n| / 2} \bar{E}_{n}^{\dagger}, & n>0, \\
0, & n \leq 0 .
\end{array}\right\}, \\
& \bar{Y}^{\dagger}\left(\bar{E}_{n}^{\dagger}\right)=\left\{\begin{array}{cc}
q^{|n| / 2} \bar{E}_{n}^{\dagger}, & n \leq 0 . \\
0, & n>0 .
\end{array}\right\} .
\end{aligned}
$$

Correspondingly,

$$
\widetilde{\mathcal{Y}}=\nu_{-} \tau_{+}\left(Y^{\dagger}\right)=q^{-1 / 4} X Y, \quad \tilde{\mathcal{Y}}^{\prime}=\nu_{-} \tau_{+}\left(Y^{\prime}\right)^{\dagger}=q^{+1 / 4} Y^{\prime} X^{-1}
$$

and we must have for $n \geq 0$ :

$$
\begin{aligned}
& \tilde{\mathcal{Y}}^{\prime}\left(\left(Y^{\prime}\right)^{n+1}\right)=q^{(n+1) / 2}\left(Y^{\prime}\right)^{n+1}, \quad \widetilde{\mathcal{Y}}^{\prime}\left(Y^{n}+q^{-n}\left(Y^{\prime}\right)^{n}\right)=0, \\
& \widetilde{\mathcal{Y}}\left(Y^{n}+q^{-n}\left(Y^{\prime}\right)^{n}\right)=q^{n / 2}\left(Y^{n}+q^{-n}\left(Y^{\prime}\right)^{n}\right), \widetilde{\mathcal{Y}}\left(\left(Y^{\prime}\right)^{n+1}\right)=0 .
\end{aligned}
$$

This is straightforward.

Generally, the map we constructed is closely connected with those that can be obtained directly from Proposition 4.2 and especially Theorem 4.8. As we see, the real source of the function $\Omega$ appears in the fact that the core subalgebra provides natural creation and annihilation operators for the $\bar{E}$-polynomials.

\section{ThE CASE OF LARGE $q$}

Let us address the spinor limit of the "second" function $G^{\checkmark}$ defined for $|q|>1$ by formula (2.15). Its theory is somewhat more involved than that for $|q|<1$.

6.1. Omega-check function. The procedure is based on the deltaversion of $R^{\checkmark}$ from (2.22) twisted as follows.

6.1.1. Twisted RE-procedure. Setting $\widetilde{\Gamma_{k}}=\Gamma_{k}^{\delta}, \widetilde{q^{k x}}=\left(q^{k x}\right)^{\delta}$, let

$$
\begin{array}{r}
\widetilde{x}(\mathcal{A}) \stackrel{\text { def }}{=}\left\{\begin{array}{c}
1 \\
t^{-1}
\end{array}\right\} \widetilde{q^{-k x}}{\widetilde{\Gamma_{k}}}^{-1} \mathcal{A} \widetilde{\Gamma_{k}} \widetilde{q^{k x}}\left\{\begin{array}{c}
1 \\
t
\end{array}\right\}, \\
\widetilde{R E}(\mathcal{A}) \stackrel{\text { def }}{=} \lim _{k \rightarrow \infty} \widetilde{x}(\mathcal{A}), \widetilde{R E}(F)=\lim _{k \rightarrow \infty}\left\{\begin{array}{c}
1 \\
t^{-1}
\end{array}\right\} \widetilde{q^{-k x}}{\widetilde{\Gamma_{k}}}^{-1}(F)
\end{array}
$$

for the operators $\mathcal{A}$ and functions $F$. The spinor constant here is directly related to the spinor constant $\widetilde{t}^{1 / 2}=\left\{t^{1 / 2}, t^{-1 / 2}\right\}$ used in the case $|q|<1$; we will return to this point below. 
6.1.2. Definition. For the function $G^{\checkmark}$ defined in (2.15),

$$
\Omega^{\vee}(X, \Lambda) \stackrel{\text { def }}{=} \widetilde{R E}\left(G^{\checkmark}\right)
$$

$$
\begin{array}{r}
=(\widetilde{\gamma}(X) \widetilde{\gamma}(\Lambda))^{-1}\left(\left\{\begin{array}{l}
1 \\
0
\end{array}\right\}+\sum_{m=1}^{\infty} q^{-m^{2} / 4}\left(\frac{\bar{E}_{-m}^{\dagger}(\Lambda)}{\prod_{s=1}^{m}\left(1-q^{-s}\right)}\left\{\begin{array}{c}
X^{-m} \\
0
\end{array}\right\}\right.\right. \\
\left.\left.+\frac{\bar{E}_{m}^{\dagger}(\Lambda)}{\prod_{s=1}^{m-1}\left(1-q^{-s}\right)}\left\{\begin{array}{c}
X^{2-m} \\
X^{-m}
\end{array}\right\}\right)\right) .
\end{array}
$$

Collecting the terms with the same products in the denominator (the norms),

$$
\times \sum_{m=0}^{\infty} \frac{q^{-m^{2} / 4}}{\prod_{s=1}^{m}\left(1-q^{-s}\right)}\left\{\begin{array}{c}
X^{-m} \bar{E}_{-m}^{\dagger}(\Lambda)+X^{1-m} q^{-m / 2-1 / 4} \bar{E}_{m+1}^{\dagger}(\Lambda) \\
X^{-m-1} q^{-m / 2-1 / 4} \bar{E}_{m+1}^{\dagger}(\Lambda)
\end{array}\right\} .
$$

With respect to $X^{-m}$,

$$
\begin{aligned}
\Omega^{\checkmark} & =(\widetilde{\gamma}(X) \widetilde{\gamma}(\Lambda))^{-1}\left(q^{-1 / 4} \bar{E}_{1}^{\dagger}\left\{\begin{array}{c}
X \\
0
\end{array}\right\}\right. \\
+ & \left.\sum_{m=0}^{\infty} \frac{q^{-m^{2} / 4}}{\prod_{s=1}^{m}\left(1-q^{-s}\right)}\left\{\begin{array}{c}
X^{-m}\left(\bar{E}_{-m}^{\dagger}+\frac{q^{-m-1}}{1-q^{-m-1}} \bar{E}_{m+2}^{\dagger}\right) \\
X^{-m}\left(1-q^{-m}\right) \bar{E}_{m}^{\dagger}
\end{array}\right\}\right) .
\end{aligned}
$$

6.1.3. Symmetrization. We claim that the spinor-symmetrization of $\Omega^{\checkmark}$ is $\left\{\mathcal{W}^{\checkmark}, 0\right\}$ for the symmetric global Whittaker function from (2.28):

$$
\begin{gathered}
\mathcal{W}^{\checkmark}(X, \Lambda)=(\widetilde{\gamma}(X) \widetilde{\gamma}(\Lambda))^{-1} \\
\times \sum_{m=0}^{\infty} q^{-\frac{m^{2}}{4}} \bar{P}_{m}\left(\Lambda ; q^{-1}\right) X^{-m} \prod_{s=1}^{m} \frac{1}{1-q^{-s}}
\end{gathered}
$$

The symmetrization is now the application of $\widehat{T}^{\checkmark}$; the latter is the $T$-symmetrizer understood as $t^{-1}+t^{-1 / 2} T$ under the limit $t \rightarrow \infty$. Since

$$
\widehat{T}^{\checkmark}=\widetilde{R E}\left(t^{-1 / 2} T\right)=\left\{\begin{array}{c}
\left(1-X^{2}\right) s+1 \\
0
\end{array}\right\}
$$

(see below), we need to check the identity:

$$
\bar{E}_{-m}^{\dagger}+\left(1-q^{-m}\right) \bar{E}_{m}^{\dagger}=\bar{P}_{m}^{\dagger} \text {, where } \bar{P}_{m}^{\dagger}=\left.P_{m}\right|_{t \rightarrow \infty}=\left.\bar{P}_{m}\right|_{q \mapsto q^{-1}}
$$

for the Rogers polynomials $P_{n}(n \geq 0)$. Indeed,

$$
P_{n}=E_{-n}+\frac{t-t q^{n}}{1-t q^{n}} E_{n} \text { due to (1.25), }
$$


which readily gives the required limit.

6.1.4. Discussion. Generally, we can conjugate the limiting procedures by any constant spinors without changing its action on diagonal operators. For instance, the Toda operator $\widetilde{R E}(\mathcal{L})$ will automatically coincide with that from (2.26). The multiplication by $\left\{1, t^{-1}\right\}$ is necessary to ensure the existence of $\Omega^{\checkmark}$ and the corresponding operators.

Theorem 2.2 (see also part (iii) of the following theorem) clarifies the appearance of this multiplier in full. Let us demonstrate it. This theorem gives that

$$
\widetilde{G}=t^{-1 / 2} \Lambda^{-1} \pi G \text { and } G^{\checkmark}=t^{1 / 2} \Lambda \pi G^{*} .
$$

The operation $R \mathbb{E}^{\delta}=\lim _{t \rightarrow 0} æ^{\delta}$ for $æ^{\delta}=\left(q^{k x} \Gamma_{k}^{-1}\right)^{\delta}$ from (5.3) can be applied to $G$. Therefore $q^{-k x} \Gamma_{k}^{-1}$ can be applied to its conjugation $G^{*}$ followed by the limit $t \rightarrow \infty$. Hence the operation

$$
\left(q^{-k x} \Gamma_{k}^{-1}\right)^{\delta}\left(t^{-1 / 2} \Lambda^{-1} \pi\right)=\Lambda^{-1} \pi\left\{\begin{array}{c}
1 \\
t^{-1}
\end{array}\right\}\left(q^{-k x} \Gamma_{k}^{-1}\right)^{\delta}
$$

can be applied to $G^{\checkmark}$ followed by the limit $t \rightarrow \infty$. Up to $\Lambda^{-1} \pi$, this is exactly the twisted (spinor) $R E$-procedure.

Recall that here $\pi=s \Gamma, \pi \Gamma_{k}^{\delta}=\Gamma_{k}^{\delta} \pi$, since $\Gamma^{\delta}$ commute with $s$ and $\Gamma$, and

$$
\left(q^{-k x}\right)^{\delta} \pi=\pi\left\{\begin{array}{c}
t^{1 / 2} \\
t^{-1 / 2}
\end{array}\right\}\left(q^{-k x}\right)^{\delta}
$$

6.2. Main theorem. Combining (3.2) and (3.12), let $\overline{\mathcal{H}}^{\varphi \dagger}$ be the span $\mathrm{C}\left[q^{ \pm 1 / 4}\right]<T^{\dagger}, \breve{\pi}, Y^{ \pm 1}>$ subject to

$$
\begin{aligned}
& T^{\dagger}\left(T^{\dagger}\right)^{\prime}=0, \breve{\pi}^{2}=1, \breve{\pi} Y \breve{\pi}=q^{-1 / 2} Y^{-1}, \\
& T^{\dagger} Y^{-1}=Y\left(T^{\dagger}\right)^{\prime} \text { for }\left(T^{\dagger}\right)^{\prime} \stackrel{\text { def }}{=} T^{\dagger}-1 .
\end{aligned}
$$

We set $X \stackrel{\text { def }}{=} \breve{\pi}\left(T^{\dagger}\right)^{\prime}$ and $X^{\prime} \stackrel{\text { def }}{=} T^{\dagger} \breve{\pi}$; then $T^{\dagger} X=X^{\prime}\left(T^{\dagger}\right)^{\prime}$ and $\left(T^{\dagger}\right)^{\prime} X^{\prime}=0=X T^{\dagger}$.

The algebra $\overline{\mathcal{H}}^{\varphi \dagger}$ is the image of the algebra $\overline{\mathcal{H}}^{\dagger}$ under the antiisomorphism $\varphi$ sending

$$
T^{\dagger} \mapsto T^{\dagger}, \pi \mapsto \breve{\pi}, X \mapsto Y^{-1}, Y \mapsto X^{\prime}, Y^{\prime} \mapsto X
$$

We will use the notations:

$$
\Omega_{\text {series }}=\widetilde{\gamma}^{\prime}(X) \widetilde{\gamma}^{\prime}(\Lambda) \Omega, \quad \Omega_{\text {series }}^{\checkmark}=\widetilde{\gamma}(X) \widetilde{\gamma}(\Lambda) \Omega^{\checkmark} .
$$


Theorem 6.1. (i) The operators

$$
\begin{aligned}
& \widehat{T}^{\checkmark}=\widetilde{R E}\left(t^{-1 / 2} T\right), \quad\left(\widehat{T}^{\prime}\right)^{\checkmark}=\widetilde{R E}\left(t^{-1 / 2} T^{-1}\right), \quad \widehat{X T}^{\checkmark}=\widetilde{R E}(X T), \\
& \widehat{X}^{\checkmark}=\widetilde{R E}\left(t^{-1 / 2} X\right), \quad\left(\widehat{X}^{\prime}\right)^{\checkmark}=\widetilde{R E}\left(t^{-1 / 2} X^{-1}\right), \\
& \widehat{Y}^{\checkmark}=\widetilde{R E}(Y), \quad\left(\widehat{Y}^{-1}\right)^{\checkmark}=\widetilde{R E}\left(Y^{-1}\right),
\end{aligned}
$$

satisfy the relations in $\overline{\mathcal{H}}^{\varphi \dagger}$ from (6.8). In particular,

$$
\left(\widehat{T}^{\checkmark}\right)^{2}=\widehat{T}^{\checkmark},\left(\widehat{T}^{\prime}\right)^{\checkmark}=\widehat{T}^{\checkmark}-1, \widehat{Y}^{\checkmark}\left(\widehat{Y}^{-1}\right)^{\checkmark}=1,\left(\widehat{X T}^{\checkmark}\right)^{2}=1 .
$$

(ii) Similar to (5.10), $\Omega^{\checkmark}=\widetilde{R E}\left(G^{\checkmark}\right)$ satisfies the relations

$$
\begin{aligned}
& \widehat{Y}^{\checkmark}\left(\Omega^{\checkmark}\right)=\Lambda^{-1}\left(\Omega^{\vee}\right), \widehat{X}^{\checkmark}\left(\Omega^{\checkmark}\right)=\bar{Y}_{\Lambda}^{\prime}\left(\Omega^{\checkmark}\right),\left(\widehat{X}^{\prime}\right)^{\checkmark}\left(\Omega^{\vee}\right)=\bar{Y}_{\Lambda}\left(\Omega^{\vee}\right), \\
& \widehat{X T}^{\checkmark}\left(\Omega^{\vee}\right)=\pi_{\Lambda}\left(\Omega^{\vee}\right), \widehat{T}^{\vee}\left(\Omega^{\vee}\right)=\bar{T}_{\Lambda}\left(\Omega^{\vee}\right),\left(\widehat{T}^{\prime}\right)^{\vee}\left(\Omega^{\vee}\right)=\left(\bar{T}^{\prime}\right)_{\Lambda}\left(\Omega^{\vee}\right),
\end{aligned}
$$

where $\bar{Y}_{\Lambda}^{\prime}, \bar{Y}_{\Lambda}, \pi_{\Lambda}, \bar{T}_{\Lambda}$ act on the argument $\Lambda$; the other operators are $X$-operators.

(iii) Following Theorem 2.2, let $\widetilde{\Omega}=\left(\Omega^{\checkmark}\right)^{*}$, where $\left(q^{1 / 4}\right)^{*}=q^{-1 / 4}$,

$$
\left(\bar{E}_{m}^{\dagger}(\Lambda)\right)^{*}=\Lambda^{-1} \bar{E}_{1-m}, \quad\left\{X^{m}, X^{n}\right\}^{*}=\left\{X^{-m}, X^{-n}\right\} \quad \text { for } m, n \in \mathrm{N} .
$$

It extends the standard conjugation considered in (3.21) from functions to spinors. Also, for $\pi$ and $\tau_{+}^{-1}(\pi)=q^{1 / 4} X^{-1} \pi$, we need their (natural) extensions to spinors:

$\pi_{\text {spin }}\left\{\begin{array}{c}X^{m} \\ X^{n}\end{array}\right\}=\left\{\begin{array}{c}q^{-\frac{n}{2}} X^{n} \\ q^{+\frac{m}{2}} X^{m}\end{array}\right\}, \Pi_{\text {spin }}\left\{\begin{array}{c}X^{m} \\ X^{n}\end{array}\right\}=\left\{\begin{array}{c}q^{\frac{1}{4}-\frac{n}{2}} X^{n-1} \\ q^{\frac{1}{4}+\frac{m}{2}} X^{m+1}\end{array}\right\}$.

Then

$$
\begin{aligned}
& \Omega(X, \Lambda)=\pi_{\text {spin }}(\Lambda \widetilde{\Omega}(X, \Lambda)) \\
& \Omega(X, \Lambda)_{\text {series }}=\Pi_{\text {spin }}\left(\Lambda \widetilde{\Omega}_{\text {series }}(X, \Lambda)\right) .
\end{aligned}
$$

6.2.1. Proof. The relations from $(i)$ hold by construction. Recall that $\widehat{X T}^{\checkmark}$ corresponds to the element $\breve{\pi}$ in $\overline{\mathcal{H}}^{\varphi \dagger}$.

Similarly, the relations for $\Omega^{\checkmark}(X, \Lambda)$ result from the definition of this function as the limit of $G^{\checkmark}$; thus it is not necessary to verify them. Nevertheless, it is instructional to check them directly using the 
following dag-Pieri rules:

$$
\begin{array}{ll}
X \bar{E}_{n}^{\dagger}=\bar{E}_{n+1}^{\dagger}-\bar{E}_{1-n}^{\dagger} & (n>0), \\
X \bar{E}_{-n}^{\dagger}=\left(1-q^{-n}\right) \bar{E}_{1-n}^{\dagger}+q^{-n} \bar{E}_{1+n}^{\dagger} & (n \geq 0), \\
X^{-1} \bar{E}_{n}^{\dagger}=\left(1-q^{1-n}\right) \bar{E}_{n-1}^{\dagger}+\bar{E}_{1-n}^{\dagger} & (n>0), \\
X^{-1} \bar{E}_{-n}^{\dagger}=\bar{E}_{-n-1}^{\dagger}+q^{-n-1} \bar{E}_{1-n}^{\dagger} & (n \geq 0) .
\end{array}
$$

For instance, we can rewrite (6.4) in a convenient compact way:

$$
\begin{gathered}
\Omega^{\checkmark}(X, \Lambda)=(\widetilde{\gamma}(X) \widetilde{\gamma}(\Lambda))^{-1}\left(q^{-1 / 4} \bar{E}_{1}^{\dagger}(\Lambda)\left\{\begin{array}{c}
X \\
0
\end{array}\right\}\right. \\
\left.+\sum_{m=0}^{\infty} \frac{q^{-m^{2} / 4}}{\prod_{s=1}^{m}\left(1-q^{-s}\right)}\left\{\begin{array}{c}
X^{-m}\left(1-q^{-m-1}\right)^{-1} \Lambda \bar{E}_{-m-1}^{\dagger}(\Lambda) \\
X^{-m}\left(1-q^{-m}\right) \bar{E}_{m}^{\dagger}(\Lambda)
\end{array}\right\}\right) .
\end{gathered}
$$

This formula is the best to check claim (iii). Actually, this claim follows from (6.7), but we prefer to give a direct verification as follows.

Upon the conjugation of $\Omega^{\checkmark}(X, \Lambda)_{\text {series }}$ and the multiplication by $\Lambda$ :

$$
\begin{aligned}
& \Lambda\left(q^{-1 / 4} \bar{E}_{1}^{\dagger}\left\{\begin{array}{c}
X \\
0
\end{array}\right\}\right)^{*}=q^{1 / 4}\left\{\begin{array}{c}
X^{-1} \\
0
\end{array}\right\}, \\
& \Lambda\left(\sum_{m=0}^{\infty} \frac{q^{-m^{2} / 4}}{\prod_{s=1}^{m}\left(1-q^{-s}\right)}\left\{\begin{array}{c}
X^{-m}\left(1-q^{-m-1}\right)^{-1} \Lambda \bar{E}_{-m-1}^{\dagger} \\
X^{-m}\left(1-q^{-m}\right) \bar{E}_{m}^{\dagger}
\end{array}\right\}\right)^{*} \\
& =\sum_{m=0}^{\infty} \frac{q^{m^{2} / 4}}{\prod_{s=1}^{m}\left(1-q^{s}\right)} \Lambda\left\{\begin{array}{c}
X^{m}\left(1-q^{m+1}\right)^{-1} \Lambda^{-1}\left(\Lambda^{-1} \bar{E}_{2+m}\right) \\
X^{m}\left(1-q^{m}\right)\left(\Lambda^{-1} \bar{E}_{1-m}\right)
\end{array}\right\} \\
& =\left\{\begin{array}{c}
(1-q)^{-1} \Lambda^{-1} \bar{E}_{2} \\
0
\end{array}\right\}+\sum_{m=1}^{\infty}\left\{\begin{array}{c}
\frac{q^{(m+1)^{2} / 4-m / 2-1 / 4}}{\prod_{s=1}^{m+1}\left(1-q^{s}\right)} X^{m} \Lambda^{-1} \bar{E}_{2+m} \\
\frac{q^{(m-1)_{2}^{2} / 4+m / 2-1 / 4}}{\prod_{s=1}^{m-1}\left(1-q^{s}\right)} X^{m} \bar{E}_{1-m}
\end{array}\right\} .
\end{aligned}
$$

Finally, applying $\Pi_{\text {spin }}$ and using that

$$
\Pi_{\text {spin }}\left\{\begin{array}{c}
A X^{m} \\
B X^{m}
\end{array}\right\}=\left\{\begin{array}{c}
B q^{-m / 2+1 / 4} X^{m-1} \\
A q^{m / 2+1 / 4} X^{m+1}
\end{array}\right\},
$$

where $A, B$ do not depend on $X$, we arrive at $\Omega(X, \Lambda)_{\text {series; }}$ claim $(i i i)$ is verified.

Using (iii), claims from (ii) can be reduced to those for $G$; see (5.9). Generally,

$$
\begin{aligned}
& A(\Omega)=B_{\Lambda}(\Omega) \Leftrightarrow A^{\vee}\left(\Omega^{\vee}\right)=B_{\Lambda}^{\vee}\left(\Omega^{\vee}\right), \text { where } \\
& A=\pi \eta\left(A^{\vee}\right) \pi, B_{\Lambda}=\Lambda\left(\eta\left(B^{\vee}\right)\right)_{\Lambda} \Lambda^{-1},
\end{aligned}
$$


where $A, B$ are elements from the corresponding nil-DAHA algebras, and $\eta$ is from (3.20). Equivalently,

$$
A^{\vee}=\pi \eta(A) \pi, \quad B_{\Lambda}^{\vee}=\Lambda(\eta(B))_{\Lambda} \Lambda^{-1},
$$

since $\eta(\pi)=\pi, \eta(X)=X^{-1}$.

Here $\pi$ must be eliminated from the formulas for $A$ and $A^{\vee}$, since it does not belong to $\overline{\mathcal{H}}^{\varphi}$ or $\overline{\mathcal{H}}^{\varphi \dagger}$ ( $\breve{\pi}$ does). Indeed, otherwise $T$ would be invertible. One can use $\pi$ in the intermediate calculation (subject to all standard identities) but the final result must not contain it. Let us demonstrate how this works for the basic operators.

The following identities give the required:

$$
\begin{aligned}
& \eta(Y)=\pi Y^{-1} \pi=q^{1 / 2} X^{-1} Y X \Rightarrow Y^{\vee}=Y^{-1},\left(Y_{\Lambda}\right)^{\vee}=q^{1 / 2} Y^{-1}, \\
& \eta(X)=X^{\prime} \Rightarrow X^{\vee}=q^{-1 / 2} X^{\prime}, \quad \eta(\pi)=\pi \Rightarrow\left(\pi_{\Lambda}\right)^{\vee}=\Lambda \pi_{\Lambda} \Lambda^{-1}, \\
& (\breve{\pi})^{\vee}=(X T)^{\vee}=\pi T X T Y^{-1}=(\pi T)(X T) Y^{-1}=Y \breve{\pi} Y^{-1}, \\
& \eta(T)=T^{\prime} \Rightarrow T^{\vee}=\pi T^{\prime} \pi=Y T^{\prime} Y^{-1}, \quad\left(T_{\Lambda}\right)^{\vee}=\Lambda T_{\Lambda}^{\prime} \Lambda^{-1} .
\end{aligned}
$$

For instance, $Y^{-1}\left(\Omega^{\vee}\right)=\left(\Lambda^{-1}\right)^{\vee}\left(\Omega^{\vee}\right)=\Lambda\left(\Omega^{\vee}\right)$. Multiplying by $Y \Lambda$, we obtain that $Y\left(\Omega^{\checkmark}\right)=\Lambda^{-1}\left(\Omega^{\checkmark}\right)$. As another example, the relations $T(\Omega)=T_{\Lambda}(\Omega)$ and $T^{\prime}(\Omega)=T_{\Lambda}^{\prime}(\Omega)$ give that

$$
\begin{aligned}
& Y T^{\prime} Y^{-1}\left(\Omega^{\vee}\right)=\Lambda T_{\Lambda}^{\prime} \Lambda^{-1}\left(\Omega^{\vee}\right) \Rightarrow T_{\Lambda}^{\prime}\left(\Omega^{\vee}\right) \\
= & Y T^{\prime} Y^{-1}\left(\Omega^{\vee}\right)=\Lambda T_{\Lambda}^{\prime} \Lambda^{-1}\left(\Omega^{\vee}\right)=T^{\prime}\left(\Omega^{\checkmark}\right) .
\end{aligned}
$$

It is equally possible to obtain these identities using the limiting procedure from $G^{\checkmark}$ or via explicit formulas for the basic operators.

\subsection{Basic operators (large q).}

6.3.1. T-check operator. Let us first obtain formula (6.6) for the image $\widehat{T}^{\checkmark}$ of the operator

$$
t^{-1 / 2} T=s+\frac{1-t^{-1}}{1-X^{2}}(s-1)=\frac{X^{2}-t^{-1}}{X^{2}-1} s-\frac{1-t^{-1}}{X^{2}-1}
$$

under $\widetilde{R E}$. Before taking the limit $t \rightarrow \infty$,

$$
\begin{gathered}
\widetilde{x}\left(t^{-1 / 2} T\right)= \\
\left\{\begin{array}{c}
1 \\
t^{-1}
\end{array}\right\} \widetilde{q^{-k x}}{\widetilde{\Gamma_{k}}}^{-1}\left(\frac{X^{2}-t^{-1}}{X^{2}-1} s-\frac{1-t^{-1}}{X^{2}-1}\right) \widetilde{\Gamma_{k} q^{k x}}\left\{\begin{array}{l}
1 \\
t
\end{array}\right\} .
\end{gathered}
$$

Here $\widetilde{\Gamma_{k}}, \widetilde{q^{k x}}$ are delta-spinors, so they commute with $s$. 
The only non-trivial commutation relations we need for this calculation and below are

$$
\begin{aligned}
& \widetilde{q^{-k x}} \Gamma \widetilde{q^{k x}}=\left\{\begin{array}{c}
t^{1 / 2} \\
t^{-1 / 2}
\end{array}\right\} \Gamma, \widetilde{\Gamma_{k}^{-1}} X \widetilde{\Gamma_{k}}=\left\{\begin{array}{c}
t^{-1 / 2} \\
t^{1 / 2}
\end{array}\right\} X, \\
& \left\{\begin{array}{c}
1 \\
t^{-1}
\end{array}\right\} s\left\{\begin{array}{c}
1 \\
t
\end{array}\right\}=\left\{\begin{array}{c}
t \\
t^{-1}
\end{array}\right\} s=s\left\{\begin{array}{c}
t^{-1} \\
t
\end{array}\right\}, \\
& \Gamma=\left\{\begin{array}{c}
\Gamma \\
\Gamma^{-1}
\end{array}\right\}, \quad X=\left\{\begin{array}{c}
X \\
X^{-1}
\end{array}\right\}, \quad s=\left\{\begin{array}{c}
s \\
s
\end{array}\right\} .
\end{aligned}
$$

When $t \rightarrow \infty$, we readily obtain that

$$
\widehat{T}^{\checkmark}=\left\{\begin{array}{c}
\left(1-X^{2}\right) s+1 \\
0
\end{array}\right\},\left(\widehat{T}^{\vee}\right)^{2}=\widehat{T}^{\checkmark},\left(\widehat{T}^{\prime}\right)^{\checkmark}=\widehat{T}^{\checkmark}-1 .
$$

6.3.2. Y-check operators. Let us present the calculation of the checkspinor Dunkl operators in detail. One has:

$$
\left\{\begin{array}{c}
1 \\
t^{-1}
\end{array}\right\} \widetilde{q^{-k x}}{\widetilde{\Gamma_{k}}}^{-1} s \Gamma\left(\frac{t^{1 / 2} X^{2}-t^{-1 / 2}}{X^{2}-1} s-\frac{t^{1 / 2}-t^{-1 / 2}}{X^{2}-1}\right) \widetilde{\Gamma_{k} q^{k x}}\left\{\begin{array}{l}
1 \\
t
\end{array}\right\} .
$$

Performing the conjugations,

$$
\begin{aligned}
& =s \Gamma\left\{\begin{array}{c}
t^{-1 / 2} \\
t^{1 / 2}
\end{array}\right\} \times\left(\frac{t^{1 / 2}\left\{\begin{array}{c}
t^{-1} \\
t
\end{array}\right\}\left\{\begin{array}{c}
X^{2} \\
X^{-2}
\end{array}\right\}-t^{-1 / 2}}{\left\{\begin{array}{c}
t^{-1} \\
t
\end{array}\right\}\left\{\begin{array}{c}
X^{2} \\
X^{-2}
\end{array}\right\}-1}\{s\right. \\
& \left.-\frac{t^{1 / 2}-t^{-1 / 2}}{\left\{\begin{array}{c}
t^{-1} \\
t
\end{array}\right\}\left\{\begin{array}{c}
X^{2} \\
X^{-2}
\end{array}\right\}-1}\right) \\
& =s \Gamma\left(\frac{\left\{\begin{array}{l}
1 \\
t
\end{array}\right\}\left\{\begin{array}{c}
X^{2} \\
X^{-2}
\end{array}\right\}-\left\{\begin{array}{c}
1 \\
t^{-1}
\end{array}\right\}}{\left\{\begin{array}{c}
t^{-1} \\
t
\end{array}\right\}\left\{\begin{array}{c}
X^{2} \\
X^{-2}
\end{array}\right\}-1} s-\frac{\left\{\begin{array}{c}
1 \\
t
\end{array}\right\}-\left\{\begin{array}{c}
t^{-1} \\
1
\end{array}\right\}}{\left\{\begin{array}{c}
t^{-1} \\
t
\end{array}\right\}\left\{\begin{array}{c}
X^{2} \\
X^{-2}
\end{array}\right\}-1}\right) .
\end{aligned}
$$$$
\widetilde{æ}(Y)
$$

\subsubsection{The symmetrization. Similarly, using that}

$$
T^{-1}=T-\left(t^{1 / 2}-t^{-1 / 2}\right)=\frac{t^{1 / 2} X^{2}-t^{-1 / 2}}{X^{2}-1} s-\left(t^{1 / 2}-t^{-1 / 2}\right) \frac{X^{2}}{X^{2}-1},
$$




$$
\begin{gathered}
\left\{\frac{\tilde{x}\left(Y^{-1}\right)=}{\left\{\begin{array}{c}
1 \\
t
\end{array}\right\} X^{2}-\left\{\begin{array}{c}
1 \\
t^{-1}
\end{array}\right\}} s-\frac{\left\{\begin{array}{l}
1 \\
t
\end{array}\right\} X^{2}-\left\{\begin{array}{c}
t^{-1} \\
1
\end{array}\right\} X^{2}}{\left\{\begin{array}{c}
t^{-1} \\
t
\end{array}\right\} X^{2}-1}\right) \Gamma^{-1} s . \\
\left\{\begin{array}{c}
t^{-1} \\
t
\end{array}\right\} X^{2}-1
\end{gathered}
$$

Finally, in the limit $t \rightarrow \infty$,

$$
\begin{aligned}
& \widehat{Y}^{\checkmark}=\Gamma^{-1}\left\{\begin{array}{c}
1 \\
1-X^{2}
\end{array}\right\}+s \Gamma\left\{\begin{array}{c}
1 \\
-X^{2}
\end{array}\right\}, \\
& \left(\widehat{Y}^{-1}\right)^{\checkmark}=\left\{\begin{array}{c}
1-X^{2} \\
1
\end{array}\right\} \Gamma+\left\{\begin{array}{c}
X^{2} \\
-1
\end{array}\right\} \Gamma^{-1} s .
\end{aligned}
$$

It is instructional to check that the product of these two operators is really 1 ; the formulas we give are adjusted to make this check almost immediate.

Since $T^{\dagger}\left(Y+Y^{-1}\right)=\left(Y+Y^{-1}\right) T^{\dagger}$ in $\overline{\mathcal{H}}^{\varphi \dagger}$ it is granted that the operator $\widehat{Y}^{\checkmark}+\left(\widehat{Y}^{\checkmark}\right)^{-1}$ preserves the space of spinors with zero second component, i.e., those fixed by $\widehat{T}^{\checkmark}$. Upon the restriction to this space,

$$
\widehat{Y}^{\checkmark}+\left(\widehat{Y}^{\checkmark}\right)^{-1}=\left\{\begin{array}{c}
\mathcal{T}^{\checkmark} \\
0
\end{array}\right\} \text { for } \mathcal{T}^{\checkmark}=\left(1-X^{2}\right) \Gamma+\Gamma^{-1}
$$

The latter is exactly the check-q-Toda operator from (2.26).

6.3.4. Pi-check operator. To complete the list of basic operators, let us provide the formula $\widehat{\pi}^{\checkmark}$, which is $\widetilde{R E}(X T)$. It is the image of $\breve{\pi}$ in the hat-dag-polynomial representation of $\overline{\mathcal{H}}^{\varphi \dagger}$ we are considering now. One has:

$$
\begin{gathered}
\widehat{\pi}^{\checkmark}=\left\{\begin{array}{c}
X \\
X^{-1}
\end{array}\right\}\left\{\begin{array}{c}
\left(1-X^{2}\right) s+1 \\
s-X^{2}
\end{array}\right\}=\left\{\begin{array}{c}
X\left(1-X^{2}\right) \\
X^{-1}
\end{array}\right\} s+\left\{\begin{array}{c}
X \\
-X
\end{array}\right\}, \\
\widehat{\pi}^{\vee}\left(\left\{\begin{array}{c}
f_{1} \\
f_{2}
\end{array}\right\}\right)=\left\{\begin{array}{c}
X\left(1-X^{2}\right) f_{2}+X f_{1} \\
X^{-1} f_{1}-X f_{2}
\end{array}\right\} .
\end{gathered}
$$

Recall that $\{A s, B s\}\left(\left\{f_{1}, f_{2}\right\}\right)=\left\{A\left(f_{2}\right), B\left(f_{1}\right)\right\}$, i.e., $s$ at the end of the component means that the remaining operator will be applied to the other component.

It is instructional to check directly that $\left(\widehat{\pi}^{\checkmark}\right)^{2}=1$ directly using the last formula from (6.23). We we will leave it as an exercise.

It is interesting to examine the operator interpretation of the relations between $\pi^{\checkmark}, T^{\checkmark}$ and $X^{\checkmark}$. The following must hold due to the general theory: 


$$
\begin{array}{r}
\left(\widehat{\pi}^{\vee}\right)\left(\widehat{T}^{\prime}\right)^{\vee}=\widehat{X}^{\vee} \stackrel{\text { def }}{=} \widetilde{B \mathbb{E}}\left(t^{-1 / 2} X\right)=\left\{\begin{array}{c}
0 \\
X^{-1}
\end{array}\right\}, \\
\left(\widehat{T}^{\vee}\right)\left(\widehat{\pi}^{\vee}\right)=\left(\widehat{X}^{\prime}\right)^{\vee} \stackrel{\text { def }}{=} \widetilde{R E}\left(t^{-1 / 2} X^{-1}\right)=\left\{\begin{array}{c}
X^{-1} \\
0
\end{array}\right\} .
\end{array}
$$

Here the calculation of operators $\widehat{X}^{\checkmark},\left(\widehat{X}^{\prime}\right)^{\checkmark}$ themselves is simple; use (6.15) and the fact that diagonal constant spinors commute with any diagonal operators. A direct verification of (6.24) is a bit more involved. For instance, let us check the second formula. The calculation goes as follows:

$$
\begin{aligned}
& \left(\widehat{T}^{\vee}\right)\left(\widehat{\pi}^{\vee}\right) \\
= & \left(\left\{\begin{array}{c}
1-X^{2} \\
0
\end{array}\right\} s+\left\{\begin{array}{c}
1 \\
0
\end{array}\right\}\right)\left(s\left\{\begin{array}{c}
X^{-1} \\
X\left(1-X^{2}\right)
\end{array}\right\}+\left\{\begin{array}{c}
X \\
-X
\end{array}\right\}\right) \\
= & \left\{\begin{array}{c}
1-X^{2} \\
0
\end{array}\right\}\left\{\begin{array}{c}
X^{-1} \\
X\left(1-X^{2}\right)
\end{array}\right\}+\left\{\begin{array}{c}
1 \\
0
\end{array}\right\}\left\{\begin{array}{c}
X\left(1-X^{2}\right) \\
X^{-1}
\end{array}\right\} s \\
& +\left\{\begin{array}{c}
1 \\
0
\end{array}\right\}\left\{\begin{array}{c}
X \\
-X
\end{array}\right\}+\left\{\begin{array}{c}
1-X^{2} \\
0
\end{array}\right\}\left\{\begin{array}{c}
-X \\
X
\end{array}\right\} s=\left\{\begin{array}{c}
X^{-1} \\
0
\end{array}\right\} .
\end{aligned}
$$

6.4. The eigenvalue problem. It is interesting to understand how the symmetries of $\Omega^{\checkmark}(X, \Lambda)$ can be obtained directly, without using the general theory. For arbitrary root systems no direct methods are known. For $A_{n}$, the Pieri formulas are essentially sufficient, although the calculations are very involved for $n>1$. Even for $n=1$ it is not immediate.

6.4.1. Preparations. Let us consider the main symmetry, which is the eigenvalue problem. It reads: $\widehat{Y}^{\checkmark}\left(\Omega^{\checkmark}\right)=\Lambda^{-1} \Omega^{\checkmark}$. We will leave the other relations from part (ii) of Theorem 6.1 as an exercise.

Let use the formula for $\widehat{Y}^{\checkmark}$,

$$
\begin{aligned}
\widehat{Y}^{\checkmark} & =\left\{\begin{array}{c}
1 \\
1-q X^{2}
\end{array}\right\} \Gamma^{-1}+\left\{\begin{array}{c}
-q^{-1} X^{2} \\
1
\end{array}\right\} \Gamma^{-1} s, \\
\widehat{Y}^{\checkmark}\left(\left\{\begin{array}{c}
f_{1} \\
f_{2}
\end{array}\right\}\right) & =\left\{\begin{array}{c}
\Gamma^{-1}\left(f_{1}\right)-q^{-1} X^{2} \Gamma^{-1}\left(f_{2}\right) \\
\left(1-q X^{2}\right) \Gamma\left(f_{2}\right)+\Gamma\left(f_{1}\right) .
\end{array}\right\}
\end{aligned}
$$


We will need both presentations of $\Omega^{\checkmark}(X, \Lambda)$, namely, that from (6.12):

$$
\begin{gathered}
\Omega^{\checkmark}(X, \Lambda)=(\widetilde{\gamma}(X) \widetilde{\gamma}(\Lambda))^{-1}\left(q^{-1 / 4} \bar{E}_{1}^{\dagger}(\Lambda)\left\{\begin{array}{c}
X \\
0
\end{array}\right\}\right. \\
\left.+\sum_{m=0}^{\infty} \frac{q^{-m^{2} / 4}}{\prod_{s=1}^{m}\left(1-q^{-s}\right)}\left\{\begin{array}{c}
X^{-m}\left(1-q^{-m-1}\right)^{-1} \Lambda \bar{E}_{-m-1}^{\dagger}(\Lambda) \\
X^{-m}\left(1-q^{-m}\right) \bar{E}_{m}^{\dagger}(\Lambda)
\end{array}\right\}\right) .
\end{gathered}
$$

and that from (6.4):

$$
\begin{aligned}
& \Omega^{\checkmark}=(\widetilde{\gamma}(X) \widetilde{\gamma}(\Lambda))^{-1}\left(q^{-1 / 4} \bar{E}_{1}^{\dagger}\left\{\begin{array}{c}
X \\
0
\end{array}\right\}\right. \\
& \left.+\sum_{m=0}^{\infty} \frac{q^{-m^{2} / 4}}{\prod_{s=1}^{m}\left(1-q^{-s}\right)}\left\{\begin{array}{c}
X^{-m}\left(\bar{E}_{-m}^{\dagger}+\frac{q^{-m-1}}{1-q^{-m-1}} \bar{E}_{m+2}^{\dagger}\right) \\
X^{-m}\left(1-q^{-m}\right) \bar{E}_{m}^{\dagger}
\end{array}\right\}\right) .
\end{aligned}
$$

Recall that $\Gamma\left(\widetilde{\gamma}(X)^{-1}\right)=q^{-1 / 4} X^{-1} \widetilde{\gamma}(X)^{-1}$ and

$$
X=\left\{X, X^{-1}\right\}, \quad \Gamma^{-1}\left(\left\{X^{-m}, X^{-m}\right\}\right)=\left\{q^{m / 2} X^{-m}, q^{-m / 2} X^{-m}\right\} .
$$

The following notations will be convenient

$$
\bar{E}_{m}^{\ddagger}=\bar{E}_{m}^{\dagger} \prod_{s=1}^{|\widetilde{n}|-1}\left(1-q^{-s}\right)^{-1},\left\{\begin{array}{c}
|\widetilde{n}|=n \text { for } n>0 \\
|\widetilde{n}|=n+1 \text { otherwise }
\end{array}\right\}
$$

see (1.20).

6.4.2. Explicit verification. Applying (6.25) to (6.27), one obtains:

$$
\begin{gathered}
\widehat{Y}^{\checkmark}\left(\Omega^{\checkmark}\right)=(\widetilde{\gamma}(X) \widetilde{\gamma}(\Lambda))^{-1} \\
\times\left(\bar{E}_{1}^{\ddagger}\left\{\begin{array}{c}
q^{-1} X^{2} \\
1
\end{array}\right\}-\left\{\begin{array}{c}
0 \\
q^{-1 / 4}\left(X^{-1}-q X\right)
\end{array}\right\}\right. \\
\left.+\sum_{m=0}^{\infty}\left\{\begin{array}{c}
q^{-(m-1)^{2} / 4} X^{1-m}\left(\bar{E}_{-m}^{\ddagger}+q^{-m-1} \bar{E}_{m+2}^{\ddagger}-q^{-1} X^{2} \bar{E}_{m}^{\ddagger}\right) \\
q^{-(m+1)^{2} / 4} X^{-1-m}\left(\left(1-q X^{2}\right) \bar{E}_{m}^{\ddagger}+\bar{E}_{-m}^{\ddagger}+q^{-m-1} \bar{E}_{m+2}^{\ddagger}\right)
\end{array}\right\}\right) .
\end{gathered}
$$

Until the end of this calculation, we will ignore certain terms involving powers of $X$ greater than or equal to -1 (but not all such terms). Correspondingly, we will use $\approx$ instead of $=$.

Since $q^{-(m-1)^{2} / 4} q^{-m} \bar{E}_{m+2}^{\ddagger}=q^{-(m+1)^{2} / 4} \bar{E}_{m+2}^{\ddagger}$, only the terms $\bar{E}_{-m}^{\ddagger}$ will contribute to the first component; the other terms will cancel each other in the final summation.

The same kind of cancelation will occur in the second component. Only $\bar{E}_{m}^{\ddagger}$ and $\bar{E}_{-m}^{\ddagger}$ will really contribute to the final summation. 
Thus,

$$
\begin{gathered}
\widehat{Y}^{\checkmark}\left(\Omega^{\vee}\right) \approx \\
(\widetilde{\gamma}(X) \widetilde{\gamma}(\Lambda))^{-1} \sum_{m=0}^{\infty}\left\{\begin{array}{c}
q^{-(m-1)^{2} / 4} X^{1-m} \bar{E}_{-m}^{\ddagger} \\
q^{-(m+1)^{2} / 4} X^{-1-m}\left(\bar{E}_{m}^{\ddagger}+\bar{E}_{-m}^{\ddagger}\right)
\end{array}\right\} .
\end{gathered}
$$

However, this expression coincides with

$$
\begin{gathered}
\Lambda^{-1} \Omega^{\checkmark}(X, \Lambda) \approx \\
(\widetilde{\gamma}(X) \widetilde{\gamma}(\Lambda))^{-1} \sum_{m=0}^{\infty} q^{-m^{2} / 4}\left\{\begin{array}{l}
X^{-m} \bar{E}_{-m-1}^{\ddagger}(\Lambda) \\
X^{-m} \Lambda^{-1} \bar{E}_{m}^{\ddagger}(\Lambda)
\end{array}\right\}
\end{gathered}
$$

due to the Pieri formula

$$
\Lambda^{-1} \bar{E}_{m}^{\ddagger}=\bar{E}_{m-1}^{\ddagger}+\bar{E}_{1-m}^{\ddagger} .
$$

It is of course with the reservation that we ignore certain powers of $X$. It holds without this reservation, which is easy to check. Generally, controlling the boundary terms in this and similar calculations is a nontrivial combinatorial problem even for $A_{n}(n>1)$. The $q$, $t$-setting and spherical polynomials manage this difficulty automatically.

\section{Appendix: Givental-Lee theory}

7.1. The J-series. Let $G$ be a complex semisimple algebraic group and let $\mathfrak{B}=G / B$ be the flag variety, where $B$ is a Borel subgroup. For $G=$ $S L(n+1)$, Givental and Lee show in [GiL] that quantum $K$-theory on $\mathfrak{B}$ produces formal series solutions to the $q$-Toda eigenvalue problem. A conjectured generalization to arbitrary semisimple $G$ is stated in [GiL], and for simply-laced $G$ this has been proved by Braverman and Finkelberg $[\mathrm{BF}]$.

Let us consider the case of $G=S L(n+1)$ in more detail and establish the connection to the $q$-Whittaker functions considered in this paper. In the notation of [GiL], the $q$-Toda difference operator is given by

$$
\widehat{H}=q^{\partial / \partial t_{0}}+q^{\partial / \partial t_{1}}\left(1-Q_{1}\right)+\cdots+q^{\partial / \partial t_{n}}\left(1-Q_{n}\right),
$$

where $Q_{i}=e^{t_{i-1}-t_{i}}$ act as multiplication operators and $q^{\partial / \partial t_{j}}: t_{i} \mapsto$ $t_{i}+\delta_{i j} \ln q$ are translations. We write $Q=\left(Q_{1}, \ldots, Q_{n}\right)$. A $q$-Whittaker function is a solution $I=I(Q, \Lambda, q)$ to the eigenvalue problem

$$
\widehat{H} I=\left(\Lambda_{0}^{-1}+\cdots+\Lambda_{n}^{-1}\right) I .
$$

Here $\Lambda=\left(\Lambda_{0}, \cdots, \Lambda_{n}\right)$ are coordinates on the maximal torus of diagonal matrices, i.e., $\Lambda_{0} \cdots \Lambda_{n}=1$. 
The $q$-Whittaker function due to Givental and Lee takes the form $I=p^{\ln Q / \ln q} J$, where $J$ is a formal power series in the variables $Q_{i}$ with coefficients in $K_{G}(\mathfrak{B}) \otimes Q(\Lambda, q)$. Here $p=\left(p_{1}, \ldots, p_{n}\right)$, and the $p_{i}$ are elements of $K_{G}(\mathfrak{B})$ represented by the pullbacks of the Hopf line bundles over the projective factors involved in the Plücker embedding of $\mathfrak{B}$. The power series $J$ is the so-called equivariant $K$-theoretic $J-$ function of $\mathfrak{B}$; it is a generating function for the $K$-theoretic one-point genus zero Gromov-Witten invariants with descendants.

In the case $n=1$, which is the main focus of this paper, the $J-$ function from [GiL] is as follows:

$$
J=\sum_{d=0}^{\infty} \frac{e^{d\left(t_{0}-t_{1}\right)}}{\prod_{m=1}^{d}\left(1-p \Lambda_{0} q^{m}\right)\left(1-p \Lambda_{0}^{-1} q^{m}\right)} .
$$

In $K_{G}(\mathfrak{B})$, the Hopf line bundle $p$ satisfies

$$
\left(1-p \Lambda_{0}\right)\left(1-p \Lambda_{0}^{-1}\right)=0 ;
$$

the factor $\mathcal{E}=p^{\ln Q / \ln q}$ is understood formally as a solution of the system

$$
q^{\partial / \partial t_{0}} \mathcal{E}=p \mathcal{E}, \quad q^{\partial / \partial t_{1}} \mathcal{E}=p^{-1} \mathcal{E}
$$

We mention that (7.3) and its $S L(3)$-generalization from [GiL] are extended to certain explicit formulas to the case of Grassmannians $S L(n+1) / P$ in $[\mathrm{T}]$.

7.2. The connection. The $q$-Whittaker function $\mathcal{W}(X, \Lambda)$ is given in (2.27) as a convergent series expressed in terms of the $q$-Hermite polynomials. This is the $B$-model interpretation of $\mathcal{W}$, as described in the Introduction. We now explain how the $J$-function enters into the Harish-Chandra asymptotic decomposition (2.29), thereby establishing the $A$-model interpretation of $\mathcal{W}$.

In view of (7.4), we consider the $J$-function upon the substitution $p=\Lambda_{0}$ or $p=\Lambda_{0}^{-1}$. Let us write $\Lambda=\Lambda_{0}$ and consider only the first substitution, as the latter is simply its conjugate under $s=s_{1}$ in the Weyl group $W$. Set $\widetilde{J}(X, \Lambda)=\left.J\left(q X^{-2}, \Lambda, q\right)\right|_{p \mapsto \Lambda_{0}}$.

Thus the variable $Q_{1}=e^{t_{0}-t_{1}}$ is replaced by $q X^{-2}$, where $X=q^{x}$. The operators $q^{\partial / \partial t_{1}}$ and $q^{\partial / \partial t_{0}}$ are replaced by $\Gamma$ and $\Gamma^{-1}$, respectively; recall that $\Gamma(f(X))=f\left(q^{1 / 2} X\right)$. The $q$-Toda operator is then given by $\mathcal{T}=\left(1-X^{-2}\right) \Gamma+\Gamma^{-1}$.

Using $\Gamma$, the relations $(7.5)$ become $\Gamma(\mathcal{E})=\Lambda^{-1} \mathcal{E}$. In terms of the Gaussian $\widetilde{\gamma}^{\prime}$ from Section 1.1.2, we take $\mathcal{E}(X, \Lambda)=\widetilde{\gamma}^{\prime}(X \Lambda) / \widetilde{\gamma}^{\prime}(X) \widetilde{\gamma}^{\prime}(\Lambda)$. It provides the leading term of the Harish-Chandra expansion of our 
global $q$-Whittaker function $\mathcal{W}(X, \Lambda)$ from (2.29). In terms of $\widetilde{J}$, the global function reads as follows. For $|q|<1$ and $|X|>|q|^{1 / 2}$,

$$
\mathcal{W}(X, \Lambda)=\langle\bar{\mu}\rangle \sum_{w \in W} \bar{\sigma}\left(w\left(\Lambda^{-1}\right)\right) \mathcal{E}(X, w(\Lambda)) \widetilde{J}(X, w(\Lambda))
$$

Note that our global function is the weighted sum of $|W|$ asymptotic solutions (here $W=\mathbf{S}_{2}$ ), corresponding to different choices of $p$ in the Givental-Lee approach. However such sums do not appear in [GiL] and there is no presence there of the $q$-Whittaker version of the celebrated Harish-Chandra $c$-function, which is $\sigma$ in (7.6). The latter is the key in the Harish-Chandra theory and its (recent) $q$-generalization.

Formula (7.6) is a limiting case of the Harish-Chandra type asymptotic decomposition of the global hypergeometric function (depending on $q, t)$ for $A_{1}$; its existence for arbitrary root systems is due to [Sto2].

\section{REFERENCES}

[BF] A. Braverman, and M. Finkelberg, Semi-infinite Schubert varieties and quantum K-theory of flag manifolds, Preprint arXiv:1111.2266 (2011). 5, 77

[Ch1] I. Cherednik, Double affine Hecke algebras, London Mathematical Society Lecture Note Series, 319, Cambridge University Press, Cambridge, 2006. 14,18

[Ch2] — , Intertwining operators of double affine Hecke algebras, Selecta Math. New ser. 3 (1997), 459-495 10

[Ch3] — , Difference Macdonald-Mehta conjecture, IMRN 10 (1997), 449-467. $4,6,15,16,18,60$

[Ch4] —, Toward Harmonic Analysis on DAHA (Integral formulas for canonical traces), Notes of the lecture delivered at University of Amsterdam (May 30, 2008); http://math.mit.edu/ etingof/hadaha.pdf 7

[Ch5] - Whittaker limits of difference spherical functions, IMRN, 20 (2009), 3793-3842; arXiv:0807.2155 (2008). 4, 19, 20, 21, 23, 24, 25, 26

[Ch6] Affine extensions of Knizhnik-Zamolodchikov equations and Lusztig's isomorphisms, in: Special functions. Proceedings of the Hayashibara forum 1990, Okayama, 63-77, Springer-Verlag (1991). 25

$[\mathrm{ChM}]$ - , and X. Ma, Spherical and Whittaker functions via DAHA II (Spinor Whittaker and Bessel functions in rank one), Selecta Mathematica (2012); Preprint arXiv: 0904.4324 (2009). 4, 5, 6, 51, 52, 53, 55, 57, 58, 59, 60, 61 
[Et] P. Etingof, Whittaker functions on quantum groups and q-deformed Toda operators, AMS Transl. Ser. 2, 194, 9-25, AMS, Providence, Rhode Island, 1999. 4,19

[FJM] B. Feigin, E. Feigin, M. Jimbo, T. Miwa, and E. Mukhin, Fermionic formulas for eigenfunctions of the difference Toda Hamiltonian, Lett. Math. Phys., 88 (2009), 39-77. 5

[GLO] A. Gerasimov, D. Lebedev, and S. Oblezin, On q-deformed $\mathfrak{g l}_{\ell+1^{-}}$ Whittaker function III, Lett. Math. Phys., 97 (2011), 1-24. 5

[GiL] A. Givental, and Y.-P. Lee, Quantum K-theory on flag manifolds, finitedifference Toda lattices and quantum groups, Invent. Math., 151 (2003), 193-219. 4, 5, 22, 77, 78, 79

[GW] R. Goodman, and N. R. Wallach, Conical vectors and Whittaker vectors, J. Functional Analysis, 39 (1980), 199-279. 22, 25

[HO] G.J. Heckman, and E.M. Opdam, Root systems and hypergeometric functions I, Comp. Math. 64 (1987), 329-352. 25

[Ion] B. Ion, Nonsymmetric Macdonald polynomials and Demazure characters, Duke Mathematical Journal 116:2 (2003), 299-318. 26

$[\mathrm{KK}] \quad$ B. Kostant, and S. Kumar, T-Equivariant K-theory of generalized flag varieties, J. Diff. Geometry 32 (1990), 549-603. 5

[Lub] D. Lubinsky, On q-exponential functions for $|q|=1$, Canad. Math. Bull. 41:1 (1998), 86-97. 7, 22

[Ma] I. Macdonald, Symmetric Functions and Hall Polynomials, Second Edition, Oxford University Press (1999). 11

[Me] M. van Meer, Bispectral quantum Knizhnik-Zamolodchikov equations for arbitrary root systems, Selecta Math., 17 (2011), 183-221. 25

[MS] — , and J.V. Stokman, Double affine Hecke algebras and bispectral quantum Knizhnik- Zamolodchikov equations, Int. Math. Res. Not., 6, (2010) 969-1040. 25

[O] E. Opdam, On the spectral decomposition of affine Hecke algebras, J. Inst. Math. Jussieu (2004). 7

[Rui] S. Ruijsenaars, Systems of Calogero-Moser type, in: Proceedings of the 1994 Banff summer school "Particles and fields", CRM series in mathematical physics, (G. Semenoff, L. Vinet, Eds.), 251-352, Springer, New York, 1999. 4, 19

[San] Y. Sanderson, On the Connection Between Macdonald Polynomials and Demazure Characters, J. of Algebraic Combinatorics, 11 (2000), 269-275. 26

[Sto1] J. Stokman, Difference Fourier transforms for nonreduced root systems, Sel. math., New ser. 9 (2003) 409-494. 60 
[Sto2] J. Stokman The c-function expansion of a basic hypergeometric function associated to root systems, Preprint arXiv:1109.0613 (2011). 4, 24, 25, 79

[Sus] S. Suslov, Another addition theorem for the q-exponential function, J. Phys. A: Math. Gen. 33: 41 (2000) L375-L380. 20

[T] K. Taipale, K-theoretic J-functions of type A flag varieties, Preprint arXiv:1110.3117 (2011). 78

[Wa] N. R. Wallach, Real Reductive Groups II, Academic Press, Boston, 1992. 22,25

(I. Cherednik) Department of Mathematics, UnC Chapel Hill, North Carolina 27599, USA, Chered@email.unc.edU

(D. Orr) Department of Mathematics, UnC Chapel Hill, North CarOLINA 27599, USA, DANORR@EMAIL.UNC.EDU 